\title{
THE BEHAVIOURAL ECOLOGY OF BOLDNESS \\ IN FISHES
}

\author{
by \\ Alexander D.M. Wilson \\ A thesis submitted to \\ The Faculty of Graduate Studies and Research \\ in partial fulfillment of the requirements for the degree of
}

\author{
Doctor of Philosophy \\ in \\ Biology \\ Department of Biology \\ Carleton University \\ Ottawa, Canada
}

April 2010

CAlexander D.M. Wilson, 2010 


$\begin{array}{ll}\begin{array}{l}\text { Library and Archives } \\ \text { Canada }\end{array} & \begin{array}{l}\text { Bibliothèque et } \\ \text { Archives Canada }\end{array} \\ \begin{array}{l}\text { Published Heritage } \\ \text { Branch }\end{array} & \begin{array}{l}\text { Direction du } \\ \text { Patrimoine de l'édition }\end{array} \\ \begin{array}{l}\text { 395 Wellington Street } \\ \text { Ottawa ON K1A ON4 } \\ \text { Canada }\end{array} & \begin{array}{l}\text { 395, rue Wellington } \\ \text { Ottawa ON K1A ON4 } \\ \text { Canada }\end{array}\end{array}$

Your file Votre référence ISBN: 978-0-494-67880-0 Our file Notre référence ISBN: $978-0-494-67880-0$

NOTICE:

The author has granted a nonexclusive license allowing Library and Archives Canada to reproduce, publish, archive, preserve, conserve, communicate to the public by telecommunication or on the Internet, loan, distribute and sell theses worldwide, for commercial or noncommercial purposes, in microform, paper, electronic and/or any other formats.

The author retains copyright ownership and moral rights in this thesis. Neither the thesis nor substantial extracts from it may be printed or otherwise reproduced without the author's permission.
AVIS:

L'auteur a accordé une licence non exclusive permettant à la Bibliothèque et Archives Canada de reproduire, publier, archiver, sauvegarder, conserver, transmettre au public par télécommunication ou par l'Internet, prêter, distribuer et vendre des thèses partout dans le monde, à des fins commerciales ou autres, sur support microforme, papier, électronique et/ou autres formats.

L'auteur conserve la propriété du droit d'auteur et des droits moraux qui protège cette thèse. $\mathrm{Ni}$ la thèse ni des extraits substantiels de celle-ci ne doivent être imprimés ou autrement reproduits sans son autorisation.
In compliance with the Canadian Privacy Act some supporting forms may have been removed from this thesis.

While these forms may be included in the document page count, their removal does not represent any loss of content from the thesis.
Conformément à la loi canadienne sur la protection de la vie privée, quelques formulaires secondaires ont été enlevés de cette thèse.

Bien que ces formulaires aient inclus dans la pagination, il n'y aura aucun contenu manquant.

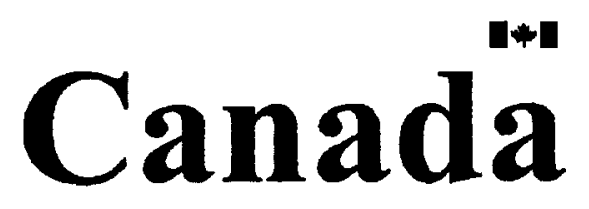




\begin{abstract}
Within populations, animals typically vary in their behaviour. Animal 'personality' or individual-level differences in behaviour that are consistent across time and/or situations has become a subject of significant research interest in recent years. Evidence for interindividual differences in personality has been steadily accumulating for a number of behavioural axes (e.g. aggression, sociability); one such axis of particular interest to behavioural ecologists is shyness-boldness. Boldness, defined as an individual's tendency to take risks, is important as it is associated with a wide range of ecologically-relevant behaviours (e.g. mating, foraging, exploration) and is exhibited by numerous species. Owing to its broad behavioural and taxonomic expression, boldness provides an ideal trait for investigating the mechanistic underpinnings of individual differences in behaviour and how they arise and are maintained in animal populations. Here, using fishes as model study systems, I investigated boldness using multiple approaches to address important and heretofore largely underappreciated areas of personality research. In Chapter 2, I used the theoretical framework of behavioural syndromes to characterize aspects of risk-taking, exploration and activity in juvenile bluegill sunfish (Lepomis macrochirus), and found that strong across-context correlations existed and at least some traits were repeatable after a 1-3 month mark-recapture experiment in the field. In Chapter 3, I characterized the relationship between boldness and intermittent locomotion in sunfish, and found that individual differences in labriform and undulatory modes of swimming were strongly correlated with willingness to take risks in a laboratory environment. In Chapter 4, I characterized the relationship between boldness in sunfish and capture technique (line angling, beach seine) in both the field and the laboratory.
\end{abstract}


Line angling in the field targeted timid individuals, as measured by laboratory boldness tests, but such a relationship was not observed in an experimental pool test. In Chapter 5, I tested whether boldness covaries with reproductive fitness correlates in female mosquitofish (Gambusia holbrooki). I found that boldness was correlated with relative fecundity but not stage of pregnancy. Collectively, my results represent novel and important contributions to further enhancing our understanding of inter-individual differences in risk-taking behaviour (boldness), and thus in personality, within populations. 


\section{ACKNOWLEDGEMENTS}

Firstly, I would like to thank my advisor Jean-Guy Godin for his excellent support and guidance throughout my Ph.D. program. His advice regarding my research and career path has been invaluable and has played, and will continue to play, an important role in my current and future successes.

Secondly, I would like to thank Sue Bertram and Ashley Ward. I greatly enjoyed our collaborations, as well as getting to know you on a more personal level. I look forward to whatever experiences together our future might hold. I also extend my gratitude to Katie Gilmour and Tom Sheratt who have been extremely helpful and supportive as members of my thesis advisory committee.

Thirdly, I would like to thank my friends and colleagues who have made my time at Carleton University so memorable. From Darwin's Bulldogs to aquatic adventures and trips into our Canadian wilderness, I have enjoyed every moment with you my friends.

Our adventures have meant more than I can adequately articulate here. For their assistance with various aspects of my research experience, I would like to give special thanks to Stacey, Tom, Keegan, and Michelle who on multiple occasions have made the impossible, possible. To my past (Kim, Kat) and current lab members (Stacey, PierreLuc, Sarah, Patrick, Adam, Rich, Heather), good friends (Tom, Dan), an awesome traveler (Michelle), marine tank buddy (Ryan), and others who are too many to name, I wish you all the best and success in every endeavour.

Lastly, I would like to thank Tiffany and my family, as without their love and support none of this would have been possible. 


\section{TABLE OF CONTENTS}

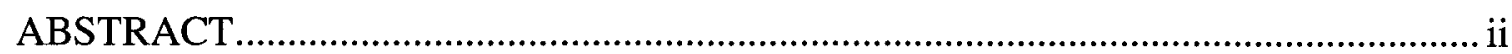

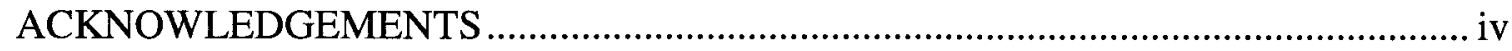

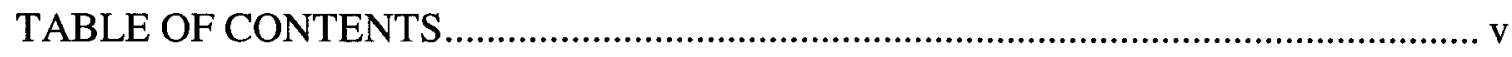

LIST OF TABLES .............................................................................................. vii

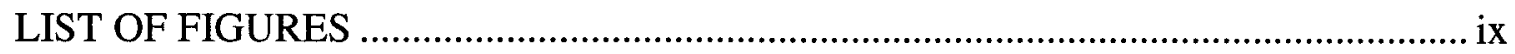

CHAPTER 1

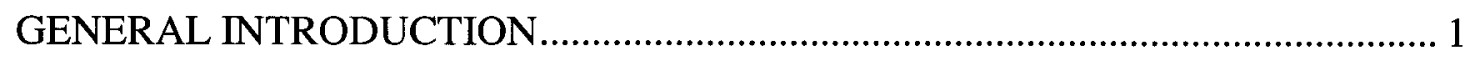

Individual-level differences in behaviour: A general overview .................................. 1

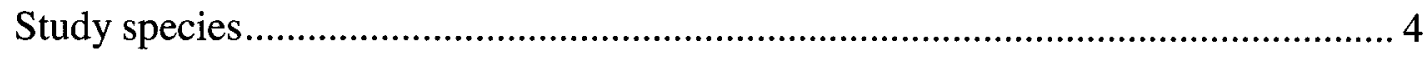

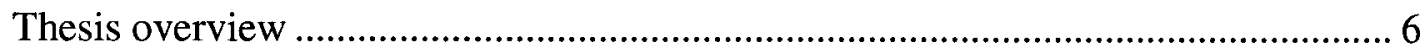

CHAPTER 2

Boldness and behavioural syndromes in the bluegill sunfish, Lepomis macrochirus ........ 9

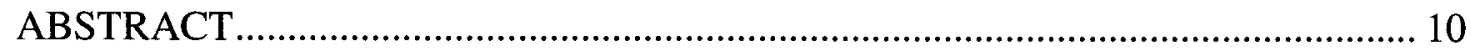

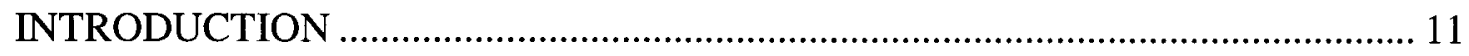

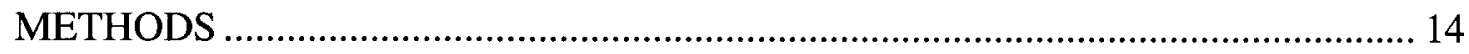

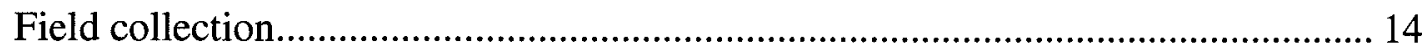

Experimental holding conditions and general experimental apparatus .................... 15

Behavioural experiments ................................................................................... 16

Repeatability of behaviour: laboratory testing of marked-recaptured fish ............... 20

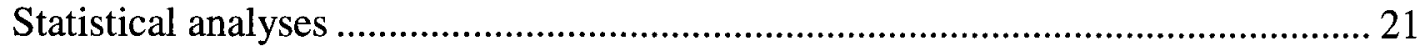

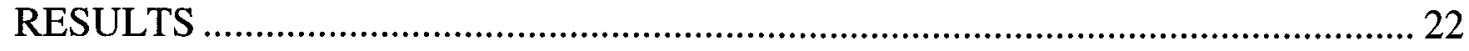

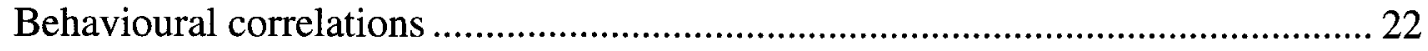

Principal components analysis (across-context syndrome analyses)........................ 23

Behavioural repeatability (mark-recapture experiment) ........................................... 23 


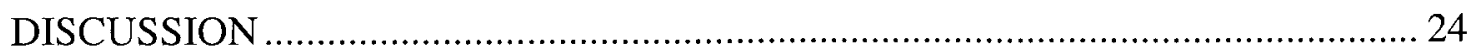

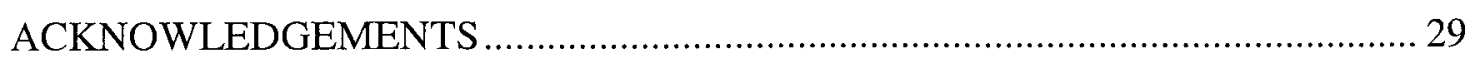

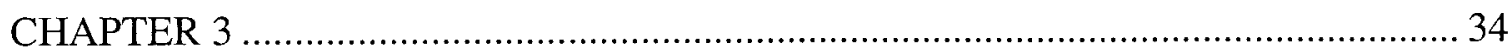

Boldness and intermittent locomotion in the bluegill sunfish, Lepomis macrochirus ${ }^{*}$... 34

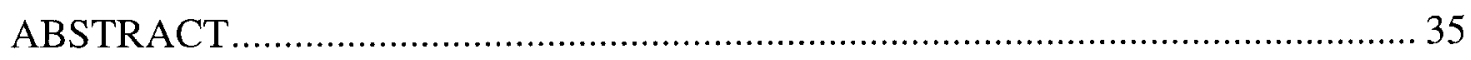

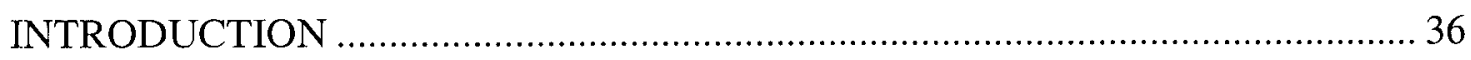

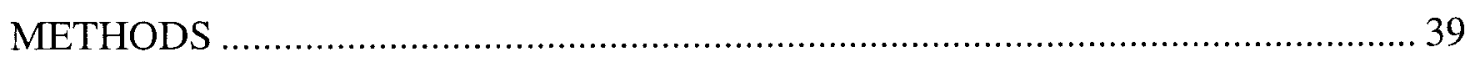

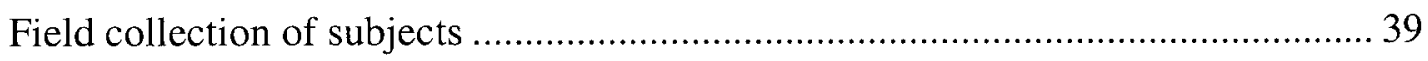

Experimental holding conditions and general experimental apparatus .................... 39

Measures of boldness (risk-taking) behaviour in a novel environment ..................... 40

Measures of intermittent locomotion ........................................................................ 41

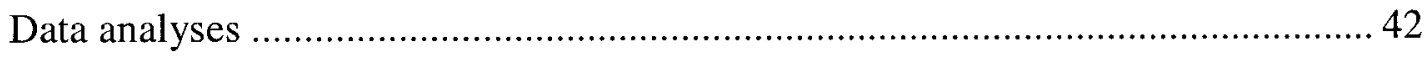

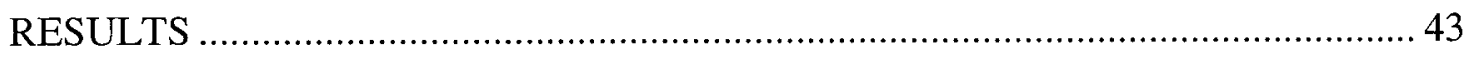

Correlations across behavioural and locomotion traits ............................................. 43

Principal components analysis of boldness and intermittent locomotion traits........ 44

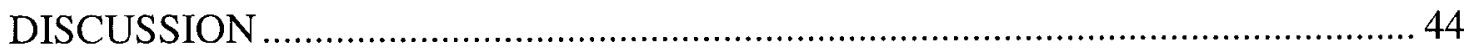

ACKNOWLEDGEMENTS …………………....................................................... 49

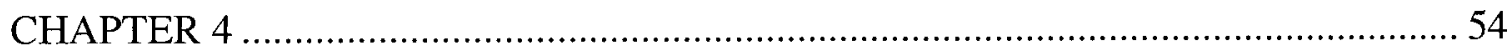

Capture technique and fish personality: recreational angling targets timid bluegill sunfish,

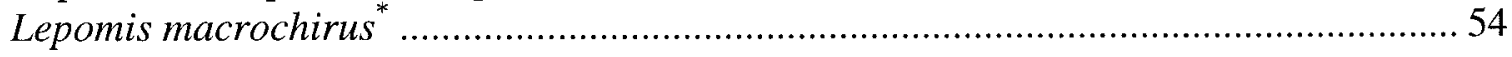

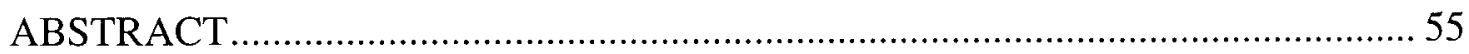

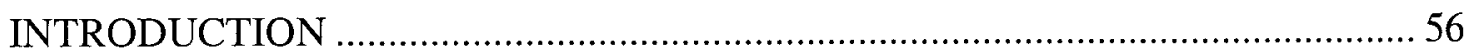

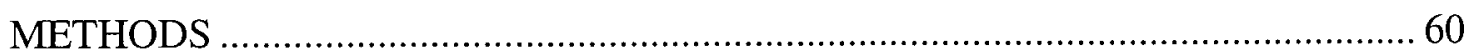

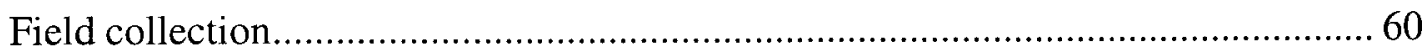

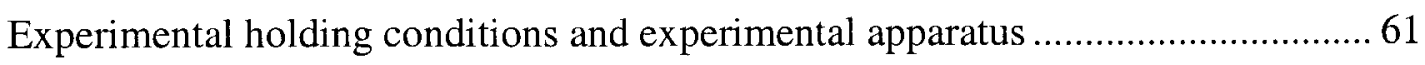

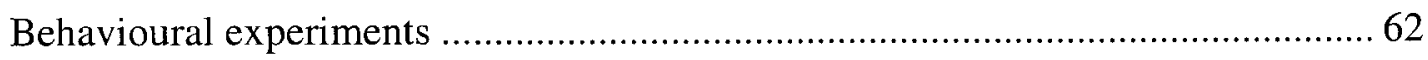

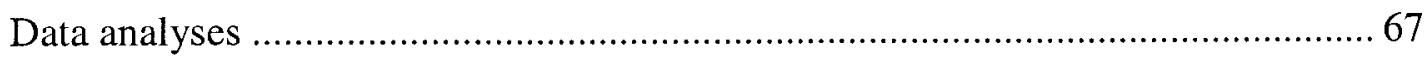

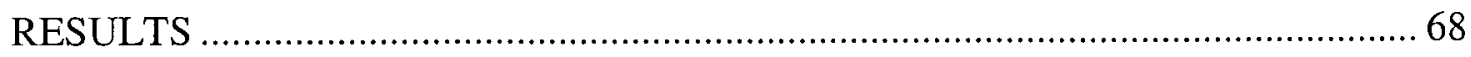

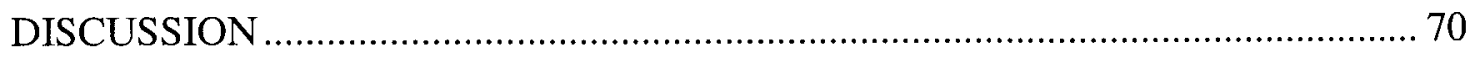

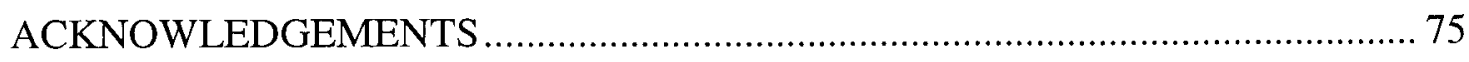


Boldness and reproductive fitness correlates in the Eastern mosquitofish, Gambusia

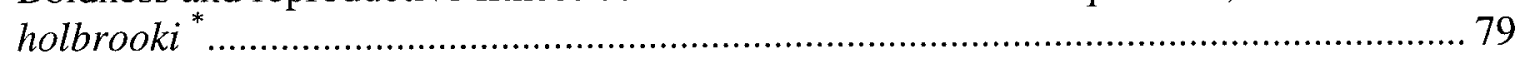

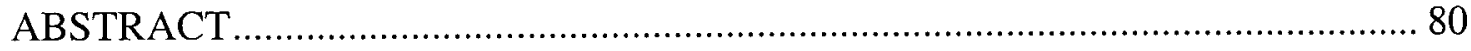

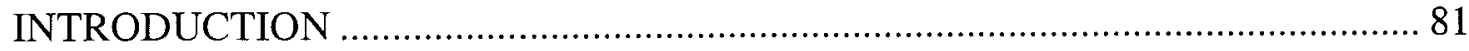

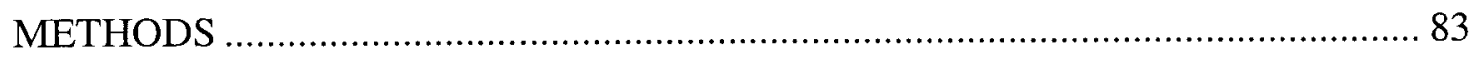

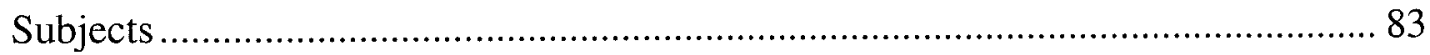

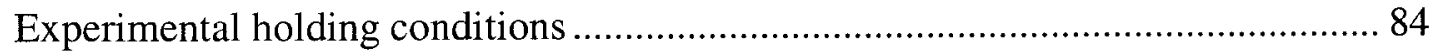

Quantifying boldness behaviour and exploratory activity .................................. 84

Quantifying morphological and reproductive traits .......................................... 88

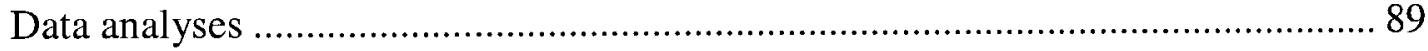

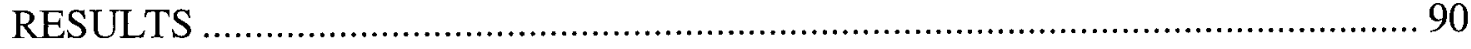

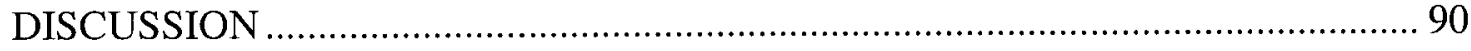

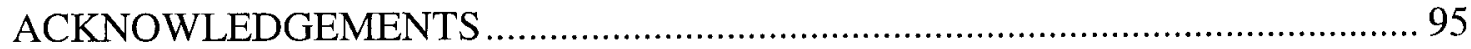

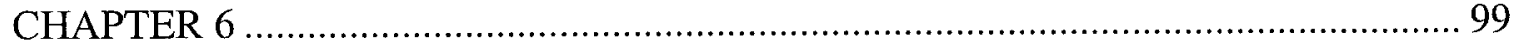

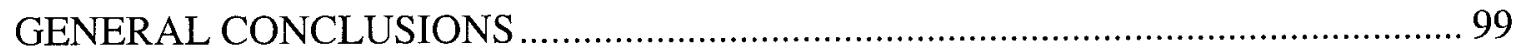

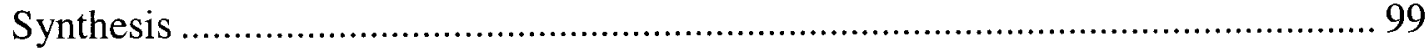

Implications and future research directions ................................................ 103

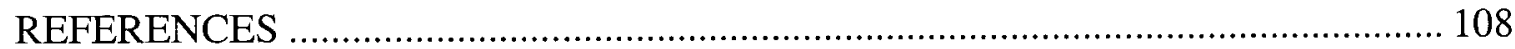




\section{LIST OF TABLES}

Chapter 2: Boldness and behavioural syndromes in the bluegill sunfish, Lepomis macrochirus

Table 2-1: PCA loadings of within-context behavioural variables used to generate a principal component scores to assess across-context correlations in activity, exploration, and risk-taking behaviour in the bluegill sunfish................................ 30

Table 2-2: Across- and within-context Spearman $\left(\mathrm{r}_{\mathrm{s}}\right)$ correlations in measures of activity, exploration and risk-taking behaviours in the bluegill sunfish...

\section{Chapter 3: Boldness and intermittent locomotion in the bluegill sunfish, Lepomis macrochirus}

Table 3-1: PCA loadings of behavioural and locomotory variables used to generate a principal component score to assess correlations between boldness and modes of intermittent locomotion in individual juvenile bluegill sunfish. ...

Chapter 4: Capture technique and fish personality: recreational angling targets timid bluegill sunfish, Lepomis macrochirus

Table 4-1: PCA loadings of behavioural variables used to generate a principal component score for water column use and other behavioural and morphological traits in juvenile bluegill sunfish

Chapter 5: Boldness and reproductive fitness correlates in the Eastern mosquitofish, Gambusia holbrooki

Table 5-1: A comparison of the stages of classification for Eastern mosquitofish embryonic development, as described by Reznick (1981)

Table 5-2: PCA loadings of behavioural variables used to generate a principal component score to assess correlations between boldness, water column use, and other behavioural, morphological, and reproductive traits in female mosquitofish. 


\section{LIST OF FIGURES}

Chapter 2: Boldness and behavioural syndromes in the bluegill sunfish, Lepomis macrochirus

Figure 2-1: Schematic representation of the experimental aquarium used on Day 3 of initial experimentation to quantify the risk-taking tendencies of individual sunfish in the presence of a realistic three-dimensional model of a largemouth bass (Micropterus salmoides).

Figure 2-2: Behavioural syndrome showing correlations between three component behaviours (activity, exploration and risk-taking) in bluegill sunfish

\section{Chapter 3: Boldness and intermittent locomotion in the bluegill sunfish, Lepomis macrochirus}

Figure 3-1: Schematic representation of the experimental aquarium used to quantify boldness and intermittent locomotion behaviours of individual juvenile bluegill sunfish in the laboratory.

Figure 3-2: Individual differences in latency to emergence from a refuge, sedentary pause duration, and time spent near the water surface in relation to undulatory and labriform swimming behaviour in juvenile bluegill sunfish.

Figure 3-3: Individual differences in modes of intermittent locomotion (relative to boldness in juvenile bluegill sunfish in a novel environment.

\section{Chapter 4: Capture technique and fish personality: recreational angling targets timid bluegill sunfish, Lepomis macrochirus}

Figure 4-1: Schematic representation of the experimental pool tank used in Experiment 2 to quantify the susceptibility of seined fish to angling practices based on their individual boldness score obtained from Experiment 1.

Figure 4-2: Mean refuge emergence, ectoparasites, general activity, time spent in upper zone of the water column, total body length, and body condition in juvenile bluegill sunfish caught by either angling or beach seining capture techniques 
Chapter 5: Boldness and reproductive fitness correlates in the Eastern mosquitofish, Gambusia holbrooki

Figure 5- 1: Observed individual differences in total body length, relative fecundity, water column use, and time spent active in relation to boldness in

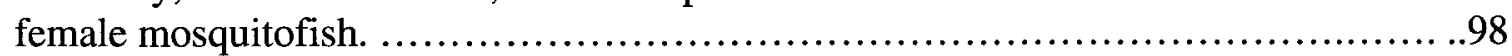




\section{CHAPTER 1}

\section{GENERAL INTRODUCTION}

This thesis is about aspects of the behavioural ecology of boldness, a component of animal "personality". The General Introduction is divided into three main sections. The first section begins with a general overview of the importance of individual-level differences in behaviour and how, until relatively recently, their importance has been underestimated by behavioural ecologists. An overview of behavioural syndromes and animal personality is provided, followed by a brief summary of the importance of boldness (a personality trait and focus my thesis research) for behavioural ecologists. The second section provides general information about the two study species in my thesis research, the bluegill sunfish (Lepomis macrochirus) and Eastern mosquitofish (Gambusia holbrooki), with an emphasis on traits that make them particularly amenable to personality research and the relevant experimental paradigm of each chapter. Finally, in the third section, I provide a brief overview of the remaining chapters of this thesis, highlighting the main objectives of each chapter as appropriate.

\section{Individual-level differences in behaviour: A general overview}

Within populations, animals typically vary in their behaviour (e.g. Wilson 1998; Foster and Endler 1999; Dall et al. 2004). From an evolutionary standpoint, this interindividual variation is difficult to explain as variation in heritable fitness-conferring traits should be

reduced by natural selection over successive generations (Sih et al. 2004). For example, if 
individuals that are more active and willing to take risks in areas of high predation are also more likely to become prey for other species and, if activity and risk-taking behaviour are heritable traits, the population should become less active and more riskaverse on average as favoured by selection over time. Yet, in spite of apparent selection pressure favouring decreased individual-level variation, behavioural diversity greater than that expected solely on differences in age, sex or body size is common in animal populations (Bolnick et al. 2003; Sih et al. 2004).

Understanding the mechanisms by which inter-individual differences in behaviour arise and persist in animal populations is one of the fundamental research challenges for behavioural ecologists. Until recently, ecologists have attempted, with limited success, to characterize such variation according to natural selection and theoretical models (e.g. optimality, population dynamics) commonly used to describe inter- and intra-specific differences in phenotype and resource use (Wilson 1998). These models, however, typically assume that animals are selected to adopt behaviours that yield the highest fitness benefit-cost ratio. These models typically fail to recognize the importance of individual-level differences in behaviour, regarding them as "ecologically equivalent" (Bolnick et al. 2003) or nonadaptive "noise" around an adaptive population mean (i.e. single optimal phenotype) (Dall et al. 2004).

The more recent theoretical framework of behavioural syndromes provides a possible mechanistic explanation for the persistence of individual-level behavioural differences within animal populations. Behavioural syndromes are defined as suites of correlated behaviours across multiple observations (Sih et al. 2004; Sih and Bell 2008). The behavioural syndrome paradigm advocates a holistic approach to studying behaviour, 
including studies across both short and long time scales and multiple behaviours concurrently. Inherent within this framework is the potential for seemingly autonomous behaviours to evolve not independently, but rather as a "suite" of correlated behaviours (Price and Langen 1992). As such, selection affecting one behavioural trait in the suite may also affect how other behaviours are expressed in different contexts/situations (Sih et al. 2004). Behaviours therefore may not be "free" to evolve to independent optima across contexts due to potential underlying physiological, behavioural or genetic constraints associated with the syndrome (Sih et al. 2004). Thus, syndrome theory may provide an explanation for the inherently high levels of individual variation observed in animal populations, as well as how seemingly maladaptive behaviours may arise and be maintained in spite of apparent selection against them. The overall significance of behavioural syndromes for behavioural ecologists is evident in the rapid accumulation of conceptual (e.g. Bell 2007; Sih and Bell 2007; Wolf et al. 2008) and empirical evidence (e.g. Bell 2005; Dingemanse et al. 2007; Wilson and McLaughlin 2007; Wilson et al. 2010a) in support of the theoretical framework initially posited by Sih et al. (2004). In addition to behavioural syndromes, the notion of animal 'personality' has also become the subject of much research interest (Réale et al. 2007). Personality is defined as individual-level differences in behaviour that are consistent across time and/or situations (Réale et al. 2007). Personality traits (e.g. boldness, aggressiveness, activity, sociability) are therefore difficult to study in isolation as they incorporate multiple types of behaviour across multiple different contexts (e.g. foraging, mating, exploration) (Bell 2007). Research on personality traits might therefore benefit from a behavioural syndrome 
approach to investigation as it too advocates a holistic approach, encompassing a breadth of ecologically relevant traits.

Boldness, defined as an individual's tendency to take risks (Wilson et al. 1994;

Réale et al. 2007), is a personality trait that has been documented in a wide range of taxa, including fishes (Ward et al. 2004; Wilson and Stevens 2005; Webster et al. 2007), birds (Dingemanse et al. 2003; Both et al. 2005), mammals (Réale and Festa-Bianchet 2003;

Martin and Réale 2008), insects (Kortet et al. 2007; Wilson et al. 2010a) and cephalopods (Sinn et al. 2006; 2008). Moreover, boldness is also associated with a number of ecologically relevant behaviours, including aggression (Johnson and Sih 2005), dispersal (Fraser et al. 2001), invasiveness (Rehage and Sih 2004), mate selection (Godin and Dugatkin 1996), foraging and antipredator behaviour (Wilson and Stevens 2005), as well as activity, exploration and space use (Wilson and McLaughlin 2007). Recent studies have also suggested that boldness may be correlated with fitness-related traits (Dingemanse et al. 2004; Sinn et al. 2006), heritable (Dingemanse et al. 2002), and subject to natural (Réale and Festa-Bianchet 2003) and 'unnatural' selection (Biro and Post 2008). As such, understanding the ecological and evolutionary implications of boldness and the mechanisms by which it arises and is maintained in animal populations represent important research challenges for behavioural ecologists.

\section{Study species}

\section{Bluegill sunfish}

The bluegill sunfish (Lepomis macrochirus) was used as a model study system for the research reported in Chapters 2-4 of this thesis. Sunfish are particularly good candidates 
for studying boldness as they occur naturally in many lake systems across southern Ontario, Canada, are amenable to a variety of capture methods (e.g. line angling, seine) (Scott and Crossman 1998), and acclimatize rapidly to laboratory conditions. Furthermore, sunfish exhibit variation in distinct morphological and behavioural traits that are correlated with individual differences in habitat and resource use (Robinson et al. 1993; Robinson and Wilson 1994; Skúlason and Smith 1995; McCairns and Fox 2004). At least one species, the pumpkinseed sunfish (Lepomis gibbosus) has previously been used as a model system to examine the context-dependency of boldness behaviour (Wilson et al. 1993; Coleman and Wilson 1998). Its congener, the bluegill sunfish, therefore offers an opportunity to investigate the nature of boldness within and between ecologically relevant behavioural contexts (see Chapter 2). In addition, the bluegill sunfish is ideal for studies investigating the relationship between boldness and locomotion, as individuals exhibit two kinematically distinct modes of swimming: labriform swimming at lower speeds and undulatory swimming at higher speeds (Jones et al. 2007; Kendall et al. 2007). Swimming mode is important to ecologists as variation in locomotion may be related to sensory processing and individual assessment of potential risk in novel environments (see Chapter 3). Lastly, bluegill sunfish exhibit dramatic changes in morphological and behavioural life-history traits in response to recreational and commercial harvesting practices (Coble 1988; Jennings et al. 1997; Beard and Essington 2000). It is thus an ideal species for studying the potential impact of anthropogenic influence on boldness in wild populations and how capture technique (e.g. line angling versus seining) may result in potential sampling biases in behavioural research (see Chapter 4). Since the bluegill sunfish is a popular sport fish across North 
America (Spotte 2007), any potential capture biases associated with individual differences in personality may have significant evolutionary and ecological consequences for the affected populations (Beard and Essington 2000; Biro and Post 2008).

\section{Eastern mosquitofish}

The Eastern mosquitofish (Gambusia holbrooki) was used as a model study system for the research reported in Chapter 5 of this thesis. Mosquitofish are known to practice internal fertilization, are livebearing and exhibit variation in boldness (Rehage and Sih 2004; Pyke 2005; Rehage et al. 2005). Furthermore, mosquitofish (Gambusia sp.) are considered to be highly invasive when introduced into novel habitats and populations have come to be established in many areas of the world by both natural and anthropogenic means (Rehage and Sih 2004; Pyke 2005). Mosquitofish are therefore ideal candidates for investigating the relationship between boldness and reproductive

fitness correlates (see Chapter 5). Due to species differences in reproductive biology (i.e. external spawning, length of time required to reach sexual maturation) and temporal limitations associated with graduate level research, it was unfeasible to investigate reproductive fitness correlates in the same manner using the bluegill sunfish.

\section{Thesis overview}

In this thesis, I investigate the behavioural ecology of boldness in fishes. In Chapter 2 (published in Behavioral Ecology 2009), I ascertained whether bluegill sunfish exhibit interindividual differences in boldness using the theoretical framework of behavioural 
syndromes. I tested wild-caught juvenile sunfish in standardized boldness experiments over several days in several different behavioural contexts. I also tested for repeatability of boldness behaviour over a 1-3 month mark-recapture period in the field.

In Chapter 3 (published in Behavioral Ecology 2010), I characterized the relationship between intermittent locomotion and boldness in bluegill sunfish. Intermittent locomotion is characterized by animals performing bursts of movement punctuated by stationary pauses of variable duration (Kramer and McLaughlin 2001). While nearly ubiquitous across taxa, no previous attempt had been made to characterize the relationship between intermittent locomotion and personality and to consider the potential ecological and evolutionary implications of such a relationship.

In Chapter 4 (under review in Behavioral Ecology), I investigated the potential relationship between boldness and capture technique in bluegill sunfish. Using line angling and seining techniques, I described the relationship between manner of capture in the wild and behavioural type (timid/bold), as characterized using boldness tests in the laboratory. As a second component of this study, I also conducted a fishing experiment in a large outdoor pool, wherein I line angled for fish previously caught by seining in a lake. This second component was meant to ascertain whether bold or timid fish were targeted similarly in an artificial pool as when captured in the field. Seined fish should represent a haphazard sample of behavioural types occurring in their natural environment, and thus not be subject to a sampling bias associated with boldness.

In Chapter 5 (published in Ethology 2010), I investigated whether boldness covaries with reproductive fitness correlates in the Eastern mosquitofish. Wild-caught females were individually tested for boldness using standardized methods and then 
dissected to ascertain whether their fecundity or stage of pregnancy was related to boldness.

Chapter 6 provides a general synthesis of my empirical results and highlights their overall contribution to the advancement of our fundamental understanding of interindividual variation in boldness and thus animal personalities. In addition, I identify some limitations of my work and discuss potential future research directions. 


\section{CHAPTER 2}

\section{BOLDNESS AND BEHAVIOURAL SYNDROMES IN THE BLUEGILL SUNFISH, LEPOMIS MACROCHIRUS ${ }^{*}$}

${ }^{*}$ Published in Behavioral Ecology

Wilson ADM, Godin J-GJ, 2009. Boldness and behavioral syndromes in the bluegill sunfish, Lepomis macrochirus. Behav Ecol 20: 231-237. 


\begin{abstract}
In recent years, evidence for inter-individual variation in "personality" within animal populations has been accumulating. Personality is defined as consistency in an individual's behavioural responses over time and/or across situations. One personality trait that has potentially far-reaching implications for behavioural ecology, and may provide insight into the mechanisms by which consistent behavioural correlations arise, is that of boldness. Boldness is defined as the tendency of an individual to take risks. Using the framework of behavioural syndromes, we tested for individual differences in boldness in the laboratory among field-caught juvenile bluegill sunfish (Lepomis macrochirus) within and across the contexts of activity, risk-taking (e.g. anti-predator), and exploratory behaviour. After such testing, individuals were tagged and returned to their lake of origin as part of a mark-recapture study testing for the repeatability of individual differences in boldness. Here, we report strong and consistent individual differences in boldness within and across all three behavioural contexts. Additionally, we observed that at least some boldness behaviours were repeatable after a 1-3 month recapture period. This study provides novel evidence for a boldness syndrome in sunfish, as well as insight into how behavioural types (e.g. shy/bold) may evolve and be maintained in natural populations.
\end{abstract}




\section{INTRODUCTION}

The behaviour of animals is known to vary widely within and between populations (Wilson 1998; Foster and Endler 1999). Understanding the origin and maintenance of such phenotypic variation has been of considerable importance to evolutionary and behavioral ecologists for many years. More recently, evidence from a number of taxa has shown that individual animals exhibit behaviours that are consistent over time and/or situations and, as such, have distinct "temperaments" or "personalities" (Réale et al. 2007). Personality traits are likely involved in many aspects of an animal's behavioural repertoire (e.g. foraging, habitat use, antipredator behaviour) (Réale et al. 2007; Martin and Réale 2008). Therefore, understanding the proximate and evolutionary mechanisms underlying these traits is of interest to behavioural ecologists (Dingemanse and Réale 2005; Réale et al. 2007). While recent studies have begun to provide insight into the proximate bases of personality traits, the fitness consequences of different behavioural types (e.g. bold/shy) and the manner by which they evolve and are maintained in natural populations remains poorly understood.

One personality axis that has potentially far-reaching implications for behavioural ecology is that of "shyness" and "boldness". Boldness may loosely be defined as the tendency of an individual to take risks and be exploratory in novel contexts (Wilson et al. 1994; Wilson and Stevens 2005). Within the shy-bold continuum, individuals vary from being extremely bold (reacting to novel stimuli by either behaving normally or becoming actively exploratory) to extremely shy (either retreating or becoming vigilant when confronted with a novel stimuli) (Wilson et al. 1993). Individual variation in boldness has been documented for a variety of taxa, including birds (Carere and van Oers 2004; Both 
et al. 2005), mammals (Réale and Festa-Bianchet 2003; Svartberg et al. 2005; Dochtermann and Jenkins 2007), reptiles (Lopez et al. 2005), fishes (Ward et al. 2004; Wilson and Stevens 2005; Bell and Sih 2007), insects (Kortet et al. 2007) and cephalopods (Sinn et al. 2006). Boldness has been shown to be associated with general activity and space use (Wilson and McLaughlin 2007), antipredator behaviour (Brown and Braithwaite 2004; Brown et al. 2005; Lopez et al. 2005), aggression (Johnson and Sih 2005), mate selection (Godin and Dugatkin 1996), learning (Dugatkin and Alfieri 2003), dispersal (Fraser et al. 2001), invasiveness (Rehage and Sih 2004), and foraging and exploration (Wilson and Stevens 2005). Furthermore, several studies have provided evidence that boldness is correlated with fitness-related traits (Dingemanse et al. 2004; Sinn et al. 2006; Brown et al. 2007a), heritable (Dingemanse et al. 2002; Drent et al. 2003; Brown et al. 2007b), and subject to natural selection (Réale and Festa-Bianchet 2003).

Until recently, behavioural ecologists have primarily relied on natural selection and optimality approaches to examine individual-level differences in behaviour, including boldness. Optimality models generally assume that animals are selected to adopt behaviours that yield the highest fitness benefit/cost ratio. However, the typical optimality approach frequently fails to recognize the importance of individual-level differences in behaviour and tends to consider such variation as nonadaptive "noise" around an adaptive population mean (or single optimal phenotype) (Dall et al. 2004). Alternatively, the recent concept of behavioral syndromes (Sih et al. 2004; Sih and Bell 2008) emphasizes individual-level responses and, at times, limited behavioural plasticity as a constraint to the evolution of optimality even when favoured by selection. 
A behavioural syndrome is defined as a suite of correlated behaviours across multiple (two or more) observations (Sih et al. 2004; Sih and Bell 2008). The concept of behavioural syndromes has increasingly been applied to wild populations (e.g. Wilson and McLaughlin 2007), and has already been extensively developed by psychologists for humans and a few domesticated animal species (Gosling 2001; Sih et al. 2004). One of the primary investigative advantages of using the concept of syndromes in behavioural research is that it advocates considering an organism's behaviour holistically, over both short and long time scales, and across different behavioural contexts or situations. Owing to the fact that boldness is associated with many behaviours (e.g. Fraser et al. 2001; Rehage and Sih 2004; Wilson and Stevens 2005), it seems appropriate to consider a syndrome approach that encompasses a range of ecologically-relevant behaviours across and within contexts, as opposed to considering behaviours independently and solely within contexts. This is particularly true as the contextual nature of boldness remains a subject of some contention, with arguments both for an adaptive domain-specific view (Coleman and Wilson 1998; Wilson and Stevens 2005; Webster et al. 2007) and a more constraint based domain-general (Johnson and Sih 2005; Dochtermann and Jenkins 2007) evolutionary view for the origin of risk-taking behaviours. Thus, the experimental framework of behavioural syndromes may provide important insight into the ecological and evolutionary implications of boldness and associated behaviours, as well as their underlying mechanistic and contextual bases (Sih and Bell 2008).

Here, we use the framework of behavioural syndromes to ascertain whether wildcaught juvenile bluegill sunfish (Lepomis macrochirus) exhibit individual variation in boldness and whether boldness behaviour is correlated across foraging, activity, space 
use, exploratory and antipredator contexts. To quantitatively assess the existence of a boldness syndrome in the bluegill sunfish, we captured juvenile individuals from a small north-temperate lake and exposed them to behavioural tests quantifying activity, exploration, and antipredatory behaviours over a 3-day period in the laboratory. Should a boldness syndrome incorporating activity, exploration and risk-taking (e.g. antipredator) behaviours exist, we predicted that boldness behaviours would be consistent and repeatable across contexts. Following laboratory trials, all fish were individually marked (where possible) and returned to their point of capture in the lake as part of a markrecapture project assessing repeatability of behavior over a 3-month period.

Repeatability is an important aspect of animal personality, as it provides insights into the consistency of individual differences in behaviour under changing environmental conditions and thus the underlying proximate (e.g. physiological, genetic) basis for the behaviour being observed. Based on the definition of personality given above, understanding the repeatability of variation in personality is key to understanding its role and importance in the ecology of wild populations (Martin and Réale 2008).

\section{METHODS}

\section{Field collection}

Between 25 June and 7 August 2007, we used a 10-m beach seine to capture 60 juvenile (Yr. 1+) bluegill sunfish (fork length: $6.2-8.3 \mathrm{~cm}$; weight: $3.55-9.17 \mathrm{~g}$ ) from the littoral zone of a small bay in Patterson Lake, Ontario, Canada $\left(44^{\circ} 34^{\prime} \mathrm{N}, 76^{\circ} 21^{\prime} \mathrm{W}\right)$. Captured individuals were placed in a cooler containing lake water (mean temperature $=23.1^{\circ} \mathrm{C}$ ) and transported to our laboratory at Carleton University (transit time $=1.5 \mathrm{~h}$ ). Ten 
individuals were captured during each collection period, once per week. Patterson Lake is a high-predation environment, as it contains many piscivorous fishes (e.g. smallmouth and largemouth bass, northern pike, pickerel) and birds (e.g. belted kingfisher, northern loon, great blue heron).

\section{Experimental holding conditions and general experimental apparatus}

Upon arrival at the laboratory, each wild-caught fish was placed singly into the "refuge" area of one of 10 glass aquaria $(82 \mathrm{~L}, 92 \times 30 \times 30 \mathrm{~cm}$, Figure 2-1). Each aquarium contained aerated and filtered dechlorinated tap water maintained at $23 \pm 1{ }^{\circ} \mathrm{C}$. All aquaria were exposed to overhead fluorescent lighting on a 12L: 12D cycle. Each aquarium was divided into thirds horizontally as well as vertically with lines drawn on the front and back walls. In doing so, each aquarium was divided into 9 distinct zones to facilitate the recording of fish water column use. The refuge area was located at one end of the aquarium, contained a corner filter and a plastic aquarium plant for cover, and was separated from the remainder of the aquarium by an opaque white plastic partition equipped with a sliding door. This gated partition was located $25 \mathrm{~cm}$ from the left side of the aquarium. All aquaria were covered externally with tan cardboard at both ends and the back wall to prevent interaction between subjects in other aquaria. In addition, all aquaria contained a $2.5 \mathrm{~cm}$ layer of natural colored aquarium gravel as a substratum.

During the holding period, each fish was fed 10 brine shrimp (Artemia sp.) per day, except on day 2 of holding (Day 1 of experimentation) as the behavioural experiment the following day involved a foraging component. Modifications to the experimental aquaria for each behavioural test are described in detail in the appropriate 
section below. To eliminate the risk of confounding our results to the novel stimulus on Days 2 and 3 of experimentation, we chose to use the same general experimental set-up as that for Day 1, except for the addition of a novel stimulus (e.g. food/object or predator model). This allowed us to both obtain a measure of behavioural consistency across days and behavioural contexts in the same arena as well as insure that the behaviour being observed was attributable to the novel within-aquarium stimulus and not the novel aquarium itself. In addition to these modifications, all aquaria were placed behind an observation blind to minimize external disturbance and to facilitate the recording of behavioural trials. All trials were recorded using a Digital 8 video camera (Sony DCRTRV280), and fish behaviours were quantified from the tapes using the behavioral analysis software JWatcher (v1.0) (Blumstein and Daniel 2007).

\section{Behavioural experiments}

\section{Day 1: latency to exit a refuge and explore a novel environment}

Approximately $24 \mathrm{~h}$ after arrival in the laboratory, individual sunfish were tested in their respective aquarium for their willingness to explore a novel environment. The experimental aquaria being used for this measure were identical to those described in the general experimental apparatus section above. At the onset of each behavioural trial, the

sliding door of the test aquarium was lifted manually via a remote pulley system operated from behind the blind, thereby allowing the fish to enter and swim freely throughout the remaining open area of the aquarium (hereafter the 'arena'). Each fish was given 60 min to exit the refuge area and explore the arena. Upon exiting the refuge, several measures of individual boldness were quantified for a further $10 \mathrm{~min}$. These boldness measures 
included individual differences in latency to emerge from the refuge area, total time spent active and total time spent in the upper third zone of the water column of the arena. Latency to exit the refuge was defined as the total time required for the focal fish to completely exit the refuge area within the $60 \mathrm{~min}$ pre-trial period. Activity was defined as the total time spent swimming (versus holding position or resting on the bottom) in the arena during the $10 \mathrm{~min}$ observation period. Both activity and water column use are increasingly being recognized as ecologically-relevant measures of risk taking and exploratory behaviour among studies of animal personality (e.g. Sih et al. 2003; Bell 2005; Wilson and McLaughlin 2007). In addition, proximity to the water surface and higher levels of activity in fishes tend to increase an individual's exposure to fish and particularly avian predators (e.g. Lima and Dill 1990; Godin 1997; Sih et al. 2003). Because we are primarily interested in measures of risk taking, behaviours were only quantified while fish were in the open arena of the aquarium, not the refuge, though time spent in the refuge after initial exit was recorded.

Following the $10 \mathrm{~min}$ trial period, the partition door was closed and each fish was returned to the refuge area of the aquarium. Fish that did not exit during the 60 min pretrial period were assigned a latency-to-exit score of $60 \mathrm{~min}$ and were excluded from all further behavioural assays (Days 2 and 3). Fish were then left undisturbed until the onset of the next experiment on Day 2.

\section{Day 2: latency to inspect a novel object/food item}

Approximately $24 \mathrm{~h}$ after the conclusion of experiment 1 (Day 1 ), the sunfish were tested individually in their respective aquarium for their willingness to explore a novel object 
and/or food item in the arena. The experimental aquaria being used for this measure were identical to those described in experiment 1 , except for the additional presentation of a novel object and food item. Approximately $5 \mathrm{~min}$ prior to the onset of behavioral testing, a piece of dried krill (1 cm long) was lowered into the experimental aquarium via a pulley system similar to that used for the partition door. The pulley system consisted of a small teardrop-shaped lead weight (novel object) attached to the most distal portion of a monofilament line, followed by the krill (novel food item) attached $15 \mathrm{~cm}$ above the weight. The food item was lowered slowly to minimize disturbance to the aquarium and suspended such that the weight just rested on the substratum and the food item was directly in the centre of the middle third of the aquarium.

Refuge door operation at the onset of each behavioural trial was identical to that described for the first experiment (Day 1). Each fish was given $60 \mathrm{~min}$ to exit the refuge area and explore the novel object/food item in the open arena. Individuals that did not exit within the pre-trial period were assigned the maximum latency-to-exit value of 60 min and held over for further experimentation on Day 3.

Upon exiting the refuge, the focal fish's latency times to touch the novel object or food item with its mouth were quantified over a 10 min trial period. Following this trial, the door to the refuge partition was lowered and the focal fish was returned to the refuge. Fish were then fed and left undisturbed until of the third experiment 3 on Day 3.

\section{Day 3: simulated predation risk}

Approximately $24 \mathrm{~h}$ after the completion of experiment 2 , the fish were tested individually for their willingness to take risks when confronted with a simulated risk of 
predation. The experimental aquaria being used for this measure was identical to those described for experiment 1 , except for the additional presentation of a realistic threedimensional model of a large (total length $25 \mathrm{~cm}$ ) piscivorous predator (largemouth bass, Micropterus salmoides) (Figure 2-1). Five minutes prior to the onset of the pre-trial period, the predator was placed $15 \mathrm{~cm}$ from the side of the experimental tank opposite the refuge. The model was positioned such that its lateral side faced the door of the refuge at the other end of the tank. Additionally, the model was suspended in midwater of the arena by monofilament line attached overhead to a clear Plexiglas rod. The rod extended from its position above the aquarium to outside of the blind such that the observer was able to produce slight lateral undulations in the model by small rotations of the rod at regular $30 \mathrm{~s}$ intervals. As with experiments 1 and 2 , focal fish were given $60 \mathrm{~min}$ to exit the refuge during the pre-trial period. Once the focal fish exited the refuge, several measures of its boldness and antipredator behaviours were quantified over a $10 \mathrm{~min}$ trial. Boldness measures quantified included total time spent active in the arena, total time spent inspecting the predator and total time spent in the zone closest to the predator model. Predator inspection was defined as an individual approaching a potential predator in a tentative, saltatory series of movements interrupted by short stationary pauses, during which time the inspector is visually fixated on the predator (Dugatkin and Godin 1992). Each inspection approach was terminated by the prey fish moving away from the predator. 


\section{Repeatability of behaviour: laboratory testing of marked-recaptured fish}

To assess the repeatability of boldness behaviour in the bluegill sunfish of Patterson Lake, we conducted a mark-recapture experiment in which all fish that completed the laboratory experiments on days 1,2 , and 3 were tagged and released at their point of capture in the lake. Tagging occurred the day after the conclusion of the 3-day experimental period using visible elastomer implant tags (Northwest Marine Technology Inc.) and following a standardized protocol (A. Ward, personal comm.). This protocol involved using unique combinations of four coloured tags and six different body locations along the dorsal musculature. Prior to tagging, fish were anesthetized using tricaine methanesulfonate (MS-222, 1:10 000 dilution) buffered with sodium bicarbonate and then injected subcutaneously with a small amount of elastomer using a syringe (29 gauge, $1 \mathrm{cc}$ ) in two locations along the dorsal fin area (e.g. left/right side of first dorsal spine). All tagged fish were then wet weighed, external parasites (e.g. blackspot, Uvulifer ambloplitis) counted, and morphometric measurements (e.g. standard length, girth) made. Following tagging, all fish were held in the laboratory for 7 days, to allow for recuperation and to ensure tag retention, before being released into their lake of origin at the same location of initial capture.

Previous studies using this type of elastomer tag have revealed $99 \%$ tag retention periods for up to 6 months (Dewey and Zigler 1996). We confirmed these findings with additional bluegill sunfish from our study site in the laboratory and found $100 \%$ tag retention and $0 \%$ mortality among 33 tagged juvenile sunfish over a 6-month period.

Marked individuals were recaptured 1-3 months after initial release (depending on release date) using a beach seine in the littoral zone of Patterson Lake. Any individuals 
that were recaptured prior to the final 2 weeks of recapturing attempts were weighed, measured and released to allow time for maximum growth over the course of the summer-early fall season. Individuals recaptured during the final 2 weeks were brought back to the laboratory in the same manner as their initial arrival, except that recaptured fish were only exposed to the third experiment (Day 3; described above) and not those conducted on Day 1 and Day 2 of initial experimentation. Four behavioural traits were quantified for repeatability $(r):(1)$ latency to exit the refuge in the presence of a predator; (2) time spent in the area of the arena closest to the predator; (3) time spent inspecting the predator; and (4) total time spent active.

\section{Statistical analyses}

First, comparisons of individual behavioural traits within and across experimental days were made using the non-parametric Spearman's rank correlation test. These correlations were made to assess individual consistency in related measures of boldness behaviour. To avoid an inflated chance of Type 1 error, our alpha level for this analysis was adjusted to be more conservative $(\mathrm{P}<0.0014)$ using the Bonferroni correction.

Second, to test for the presence of a behavioural syndrome incorporating activity, risk-taking and exploratory behaviour contexts, individual traits that best represented each of these behavioural contexts were collapsed into first principal component scores for each axis of interest using Principal Components Analysis (PCA) (Table 2-1). Across-context correlations between these scores were then calculated using the Spearman's rank correlation test as above. To avoid an inflated chance of Type 1 error, 
our alpha level for this second analysis was also adjusted to be more conservative $(\mathrm{P}<$ 0.0166) using the Bonferroni correction.

Lastly, to quantify behavioural repeatability $(r)$ in recaptured individuals, we followed the protocol described in Lessells and Boag (1987). Data were normalized by $\log$ transformation, where necessary. To establish the significance of our measures of repeatability, we calculated the $95 \%$ confidence intervals for each measure as recommended by Nakagawa and Cuthill (2007).

\section{RESULTS}

All pair-wise correlations that were significant, or suggest strong trends $(P<0.05)$, are shown in Table 2-2. Only those correlations that were significant at the appropriate adjusted alpha level (see Methods) are discussed in further detail below.

\section{Behavioural correlations}

In general, individuals that were more exploratory and emerged faster from the refuge had shorter latencies to touch a novel object (following exit from refuge) and were more active than less exploratory individuals. Individuals that were more exploratory and more active were also more willing to take risks by spending longer periods of time near the surface and closer to the predator model than less active/exploratory individuals. Similarly, those more active individuals also spent more time in the zone closest to the predator and spent more time inspecting the predator than less active individuals (Table 2-1). 


\section{Principal components analysis (across-context syndrome analyses)}

Spearman rank correlation tests between the first PCA scores for all three axes of behaviour suggest strong across context-correlations between activity, risk-taking and exploratory behaviours. In particular, risk taking was strongly positively correlated with activity $\left(\mathrm{r}_{\mathrm{s}}=0.615, \mathrm{P}<0.001\right.$, Figure 2-2). Individuals that were more active were also more willing to take risks when confronted with a potential predation threat. Similarly, exploratory behaviour was strongly negatively correlated with both activity $\left(r_{s}=-0.614\right.$, $P=0.003$, Figure 2) and risk taking $\left(r_{s}=-0.564, P=0.008\right.$, Figure 2-2), suggesting that more exploratory individuals (e.g. shorter latency to exit refuge) were also more active and spent more time performing risky behaviours than less exploratory individuals.

\section{Behavioural repeatability (mark-recapture experiment)}

During the course of our field season, 19 of a total of 43 marked and released individuals were recaptured. Six of these individuals were released prior to our final collection period in an attempt to allow for maximal growth during the field season and were not recaptured. The remaining 13 fish were recaptured in the final collection period of the study and were brought back to the laboratory to test for repeatability of their exploratory/antipredator behaviour previously exhibited on Day 3 of the initial laboratory experiments (on average $36 \pm 4$ days earlier). Of the four behavioural traits considered, only latency to exit the refuge $(r=0.63,95 \% \mathrm{CI}=0.15-1.33)$ and time spent in the area of the arena closest to the predator $(r=0.83,95 \% \mathrm{CI}=0.59-1.77)$ were repeatable. In contrast, time spent being active $(r=-0.15,95 \% \mathrm{CI}=-0.74-0.44)$ and time spent 
inspecting the predator $(r=-0.20,95 \% \mathrm{CI}=-0.7894-0.3924)$ were not significantly repeatable.

\section{DISCUSSION}

Our results showed that juvenile bluegill sunfish exhibited consistent and repeatable individual differences in behaviour and that these differences were reflected in a behavioural syndrome incorporating aspects of activity, risk-taking (e.g. anti-predator) and exploratory behaviour. We characterize the observed behavioural syndrome as being a boldness syndrome because individual willingness to take risks (e.g. predator inspection) and explore novelty are significantly linked across contexts. Generally, bolder individuals were more active, more willing to explore novel objects/environments and more willing to inspect a potential predator and spend time in risky areas than shy individuals.

Our study addresses three important areas of current research need, namely animal personality, behavioural consistency/repeatability, and the nature of behavioural syndromes in wild-caught animals. As predicted, using pair-wise correlation analysis, we found strong behavioural correlations across contexts, across individual traits and, more importantly, across each of the principal component scores representing the three behavioural axes of interest (e.g. exploration, activity and risk taking). These results provide compelling evidence for behavioural correlations across multiple observations and thus for behavioural syndromes (sensu Sih et al. 2004; Sih and Bell 2008) in bluegill sunfish. In addition, the individual behavioural traits that we used as a basis for our PCA analyses also demonstrated strong correlations, and therein consistency, in multiple 
measures of similar behaviours (Table 2-2). One such behaviour that appears to play a prominent role in this boldness syndrome is that of emergence behaviour. Emergence from a refuge into an open habitat carries a certain level of risk of predation and uncertainty about ambient ecological conditions in that habitat (Godin 1997; Sih 1997). Similarly, although latency to emerge from a shelter is commonly used as a laboratory measure of animal personality (Hedrick 2000; Brown and Braithwaite 2004; Lopez et al. 2005; Wilson and McLaughlin 2007), the importance of this behaviour and its relationship to fitness remains unclear, though some studies do exist (e.g. Brown et al. 2005; Brown et al. 2007a). For example, Brown et al. (2007a) found that boldness behaviours, including refuge emergence, were strongly influenced by both body size and predation pressure in the poeciliid fish Brachyraphis episcopi. In our study, latency to emerge from a refuge was highly correlated across all three experimental days as well as with activity, novel object exploration, water column position and time spent in close proximity to the predator model. We also found that emergence behaviour was highly repeatable over the long term based on our mark-recapture study. The fact that emergence behaviour was consistent across all three experimental days, as well as repeatable among those fish recaptured, suggests that this behaviour has some ecological and/or evolutionary importance.

From an adaptive standpoint, poor or uncertain information about the environment outside a refuge pose a serious constraint on the ability of potential prey to respond adaptively to ambient conditions, as represented for example by food availability, predation risk and novel situations (e.g. new environments), outside of its refuge (Sih 1992, 1997). Alternatively, emergence behaviour may represent some 
underlying genetic predisposition arising through natural selection pressures favouring it (e.g. independent selection) or other traits that it may be correlated with (e.g. correlational selection). Bell (2005) demonstrated experimentally that selection regimes, such as predation, can favor the development of behavioural syndromes. As such, activity, risk-taking and exploratory behaviour in bluegill sunfish may similarly be shaped by high predation intensity at our study site (Patterson Lake). Correlations between activity and exploratory behaviour have been shown to be relatively common across species, lending support to the notion that this correlation is shaped by natural selection (Sih et al. 2004; Réale et al. 2007; Biro and Post 2008). This proposition is further supported by recent studies that have shown that boldness behaviour can have a genetic component (Wright et al. 2006) and may therefore be heritable in some species (Drent et al. 2003; Sinn et al. 2006; Brown et al. 2007b).

The importance of predation in shaping risk-taking behaviour associated with boldness was a primary consideration in our choosing to repeat the simulated predator experiment as a test of behavioural repeatability. As such, we expected antipredator behaviour to remain temporally consistent over the course of our 3-month experimental period due to apparently high predation pressures present at our field site for the duration of the study. Interestingly, we found that only emergence behaviour and time spent near the predator model were repeatable. Neither activity level nor predator inspection was repeatable. There are several plausible explanations for these differences. Firstly, as advocated by Bell and Stamps (2004), these differences may reflect adaptive temporal variation in trait combinations that are influenced by variation in developmental and/or ecological circumstances over time. Although difficult to assess in one population over a 
short time period, Dingemanse et al. (2007) have provided evidence for the adaptive evolution of favourable trait combinations with an inter-population comparison of the three-spined stickleback (Gasterosteus aculeatus).

Alternatively, it is also possible that our non-significant repeatability findings above may be a result of a sample size effect or, more likely, habituation. Juvenile sunfish demonstrate high levels of learning and rapidly recognize previously encountered stimuli (Gotceitas and Colgan 1988; Colgan et al. 1991; Kieffer and Colgan 1992). It is therefore possible that our recaptured fish remembered their initial encounter with the predator model (on Day 3 of the initial experiments) and no longer considered it a significant threat when retested several weeks later. As such, their behaviour (activity, predator inspection) toward a novel threat stimulus and one to which they have been acclimated would likely be different, as we observed and has been observed in other fish species (e.g. Brown 2001). In contrast, the observed repeatability of refuge emergence behaviour might be attributable to its importance in the first critical moments of information gathering when exiting a refuge, prior to predator stimulus recognition.

In addition to consistency, repeatability of behaviour, the nature of behavioural correlations across contexts, and the link between personality and fitness remain topics in need of further research. A recent meta-analysis of personality traits suggests that boldness (in males) appears to favour increased reproductive success at the expense of long-term survival (Smith and Blumstein 2008). While the underlying mechanistic basis for this trade-off remains unclear, Smith and Blumstein (2008) suggest that both shy and bold behavioural types are maintained in natural populations because of equal respective lifetime reproductive success. For example, shy individuals might experience a short- 
term reproductive disadvantage compared to bold individuals but, because of their greater longevity, their overall life-time reproductive success might be similar to that of bold individuals. This pattern, though not explicitly tested, is consistent with other current models explaining the evolution and maintenance of personality differences among individuals in populations (Bell and Stamps 2004; Stamps 2007; Wolf et al. 2007). Additionally, any fitness differences between bold and shy individuals may shed some light on the origin and maintenance of the behavioural differences evident between different reproductive and life-history strategies, such as that exhibited between parental and cuckolder males in fishes (Gross 1982), and perhaps even the ecological diversification seen in resource polymorphisms (Skúlason and Smith 1995; Wilson and McLaughlin 2007).

Our current study illuminates for the first time the nature of a boldness syndrome in bluegill sunfish and the temporal repeatability and consistency of the behaviours involved. More research is needed to better understand the ecology and evolution of such a behavioural syndrome in sunfish in particular and in other animals in general. Though not presented formally here, we did quantify parasite load, hepatic-somatic index, and instantaneous growth rates in our recaptured sunfish and found that some fitness traits were correlated, even with our small sample size (Wilson and Godin unpublished data). However, the connection between these fitness correlates and boldness behaviour remains unclear and is currently the subject of a larger ongoing study (Chapters 4 and 5 , and A.D.M. Wilson and T.R. Binder, unpublished data). 


\section{ACKNOWLEDGEMENTS}

We thank R. Bell for permission to access Patterson Lake and the Ministry of Natural Resources of Ontario for the necessary fish collection permit. Our study was approved by the institutional Animal Care Committee at Carleton University (protocol B06-7) and thus adheres to the guidelines of the Canadian Council on Animal Care and the laws of Canada. Funding: Natural Sciences and Engineering Research Council of Canada (NSERC) Discovery Grant (J.-G.J.G.); NSERC Postgraduate Scholarship (A.D.M.W.); Carleton University graduate studentship (A.D.M.W.). 
Table 2-1: PCA loadings of within-context behavioural variables used to generate a principal component scores (PC1) to assess across-context correlations in activity, exploration, and risk-taking behaviour in the bluegill sunfish ( $\mathrm{D}=\mathrm{Day})$.

\begin{tabular}{lllc}
\hline Behavioural context & Behaviours within each context & $\begin{array}{c}\text { Loadings for } \\
\text { PC1 }\end{array}$ & $\begin{array}{c}\% \\
\text { variation } \\
\text { explained }\end{array}$ \\
& & & 44.7 \\
\hline Exploration & Latency to emerge from refuge (D1) & 0.6608 & \\
& Latency to emerge from refuge (D2) & 0.5612 & \\
& Latency to emerge from refuge (D3) & 0.4843 & \\
& Latency to touch object (D2) & 0.1179 & \\
\hline Activity & Activity (D1) & 0.7071 & 51.2 \\
& Activity (D3) & 0.7071 & \\
\hline Risk-taking & Time spent in upper water zone (D1) & 0.3517 & 50.4 \\
& Time spent close to predator (D3) & 0.6804 & \\
& Time spent inspecting predator (D3) & 0.6429 & \\
\hline
\end{tabular}


Table 2-2: Across- and within-context Spearman $\left(\mathrm{r}_{\mathrm{s}}\right)$ correlations in measures of activity, exploration and risk-taking behaviours in the bluegill sunfish. All Spearman correlations at $\mathrm{P}<0.05$ are shown; correlations that are significant at Bonferroni's adjusted alpha $=$ 0.0014 are marked with an *. $\mathrm{N}$ denotes number of fish tested.

\begin{tabular}{|c|c|c|c|c|}
\hline Behavioural Trait 1 & Behavioural Trait 2 & $r_{s}$ & $P$-value & $\mathrm{N}$ \\
\hline Latency to emerge (Day 1) & $\begin{array}{l}\text { Time spent in upper } \\
\text { water zone (Day 1) }\end{array}$ & -0.458 & $0.0012^{*}$ & 47 \\
\hline Latency to emerge (Day 1) & Activity (Day 1) & -0.592 & $<0.0001^{*}$ & 47 \\
\hline Activity (Day 1) & $\begin{array}{l}\text { Time spent in upper } \\
\text { water zone (Day 1) }\end{array}$ & 0.483 & $0.0006^{*}$ & 47 \\
\hline Latency to emerge (Day 1) & Latency to emerge (Day 2) & 0.474 & $0.0009 *$ & 46 \\
\hline Latency to emerge (Day 2) & $\begin{array}{l}\text { Time spent in upper } \\
\text { water zone (Day 1) }\end{array}$ & -0.369 & 0.0116 & 46 \\
\hline Latency to emerge (Day 2) & Activity (Day 1) & -0.427 & 0.0031 & 46 \\
\hline $\begin{array}{l}\text { Latency to touch novel } \\
\text { object/food item (Day 2) }\end{array}$ & Latency to emerge (Day 1) & 0.450 & 0.0356 & 22 \\
\hline Latency to emerge (Day 3) & $\begin{array}{l}\text { Time spent in upper } \\
\text { water zone (Day } 1 \text { ) }\end{array}$ & -0.305 & 0.0439 & 44 \\
\hline Latency to emerge (Day 3) & Latency to emerge (Day 1) & 0.497 & $0.0006^{*}$ & 44 \\
\hline Latency to emerge (Day 3) & Latency to emerge (Day 2) & 0.524 & $0.0003^{*}$ & 44 \\
\hline Activity (Day 3) & Latency to emerge (Day 2) & -0.313 & 0.0460 & 41 \\
\hline Activity (Day 3) & Latency to emerge (Day 3) & -0.408 & 0.0081 & 41 \\
\hline $\begin{array}{l}\text { Time spent close to } \\
\text { predator (Day 3) }\end{array}$ & Latency to emerge (Day 2) & -0.415 & 0.0079 & 41 \\
\hline $\begin{array}{l}\text { Time spent close to } \\
\text { predator (Day } 3 \text { ) }\end{array}$ & Activity (Day 3) & 0.795 & $<0.0001^{*}$ & 41 \\
\hline $\begin{array}{l}\text { Time spent inspecting } \\
\text { predator (Day } 3 \text { ) }\end{array}$ & Activity (Day 3) & 0.569 & $0.0001^{*}$ & 41 \\
\hline $\begin{array}{l}\text { Time spent inspecting } \\
\text { predator (Day } 3 \text { ) }\end{array}$ & $\begin{array}{l}\text { Time spent close to } \\
\text { predator (Day 3) }\end{array}$ & 0.610 & $<0.0001 *$ & 41 \\
\hline
\end{tabular}




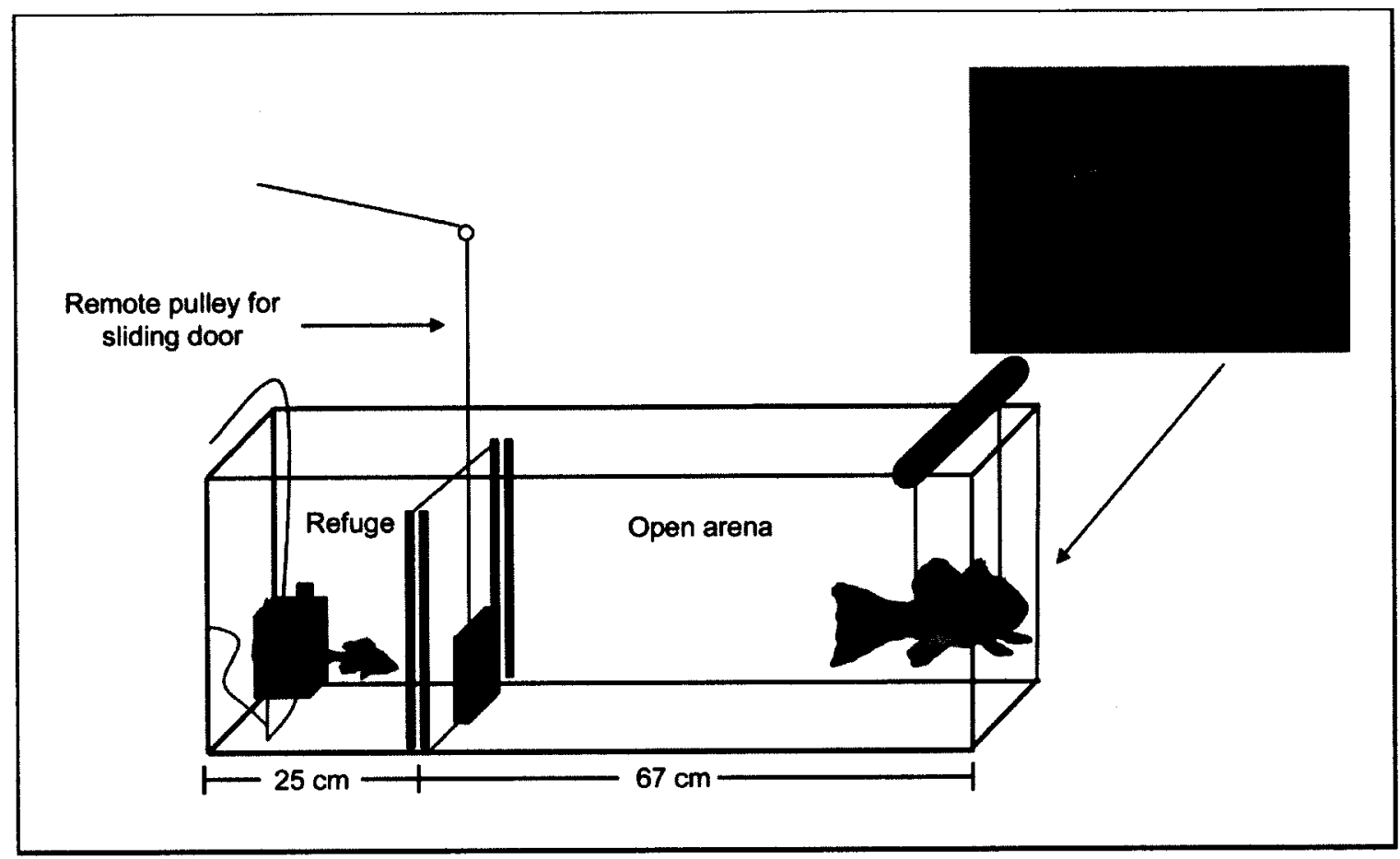

Figure 2-1: Schematic representation of the experimental aquarium used on Day 3 of initial experimentation to quantify the risk-taking tendencies of individual sunfish in the presence of a realistic three-dimensional model of a largemouth bass (Micropterus salmoides). The same experimental set-up was used in experiments 1 (Day 1) and 2 (Day 2), except that the predator model was excluded. 


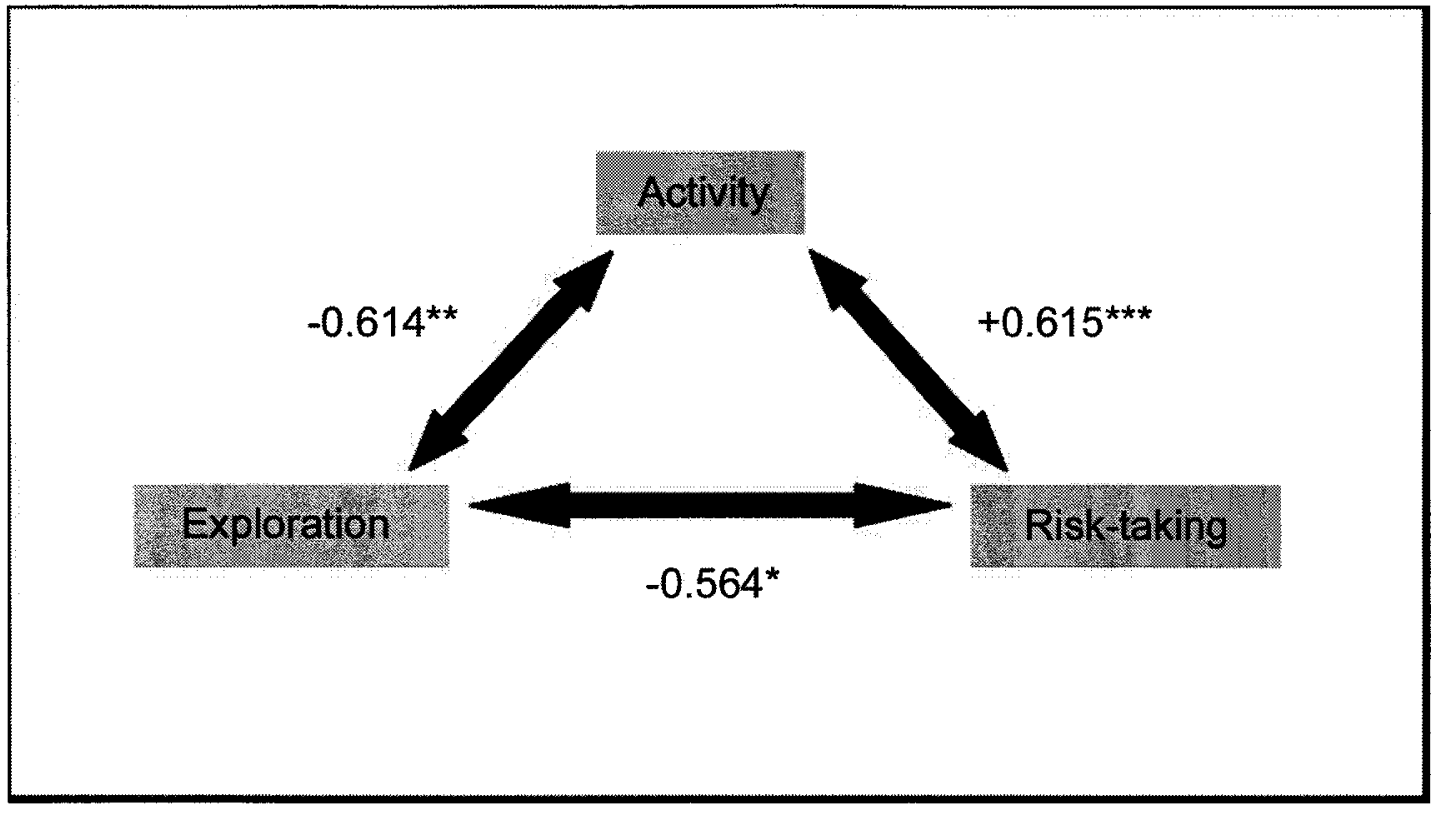

Figure 2-2: Behavioural syndrome showing correlations between three component behaviours (activity, exploration and risk taking) in bluegill sunfish. Numbers shown indicate results from Spearman rank $\left(r_{s}\right)$ correlations tests. Level of significance are indicated by $(*)$ for each pair of behaviours $(* \mathrm{P}=0.008 ; * * \mathrm{P}=0.003 ; * * * \mathrm{P}<0.001)$. 


\section{CHAPTER 3}

\section{BOLDNESS AND INTERMITTENT LOCOMOTION IN THE BLUEGILL SUNFISH, LEPOMIS MACROCHIRUS *}

$*$

Published in Behavioral Ecology

Wilson ADM, Godin J-GJ, 2010. Boldness and intermittent locomotion in the bluegill sunfish, Lepomis macrochirus. Behav Ecol 21: 57-62. 


\begin{abstract}
Intermittent locomotion, characterized by moves interspersed with pauses, is a common pattern of locomotion in animals, but its ecological and evolutionary significance relative to continuous locomotion remains poorly understood. Although many studies have examined individual differences in both intermittent locomotion and boldness separately, to our knowledge, no study to date has investigated the relationship between these two traits. Characterizing and understanding this relationship is important, as both locomotion and boldness are associated with several ecologically-relevant behaviours such as foraging, mating, predator evasion, exploration and dispersal. Here, we report on individual differences in boldness (risk-taking behaviour) and intermittent locomotion in a novel laboratory environment in field-caught juvenile bluegill sunfish (Lepomis macrochirus). Our results show that juvenile bluegill sunfish exhibited individual-level variation in two modes of intermittent locomotion (undulatory and labriform swimming) and that this variation was correlated with differences in their boldness behaviour. Generally, bolder individuals spent more time moving fast, for longer durations and with shorter pauses than more timid individuals. Neither boldness nor locomotion was correlated with body size or body condition. This study provides the first empirical evidence for a link between an animal 'personality' trait and intermittent locomotion.
\end{abstract}




\section{INTRODUCTION}

Animals exhibit diverse patterns of locomotion, depending in part on habitat, ecological context, body form and ontogenetic stage (e.g. McAdam and Kramer 1998; Kramer and McLaughlin 2001; Higham 2007). Understanding the nature of inter-individual variation in animal locomotion is of considerable importance to evolutionary and behavioural ecologists as many important behaviours, including foraging, mating, predator evasion, exploration and dispersal, all necessitate some level of movement. Until recently, most studies on animal locomotion have implicitly assumed that movement is steady-state, involving long bouts of continuous movement at relatively slow speeds (reviewed in Kramer and McLaughlin 2001). However, most animals instead move by performing bursts of movement punctuated by stationary pauses of variable duration (Kramer and McLaughlin 2001). Such a pattern of locomotion is referred to as intermittent or saltatory locomotion (Kramer and McLaughlin 2001; McLaughlin and Grant 2001; Trouilloud et al. 2004).

Intermittent locomotion is widespread taxonomically and has been documented in numerous invertebrate and vertebrate species (reviewed in Kramer and McLaughlin 2001). Further, this pattern of locomotion is strongly associated with individual differences in general activity, exploration, antipredator or vigilance behaviour (Trouilloud et al. 2004), foraging (McLaughlin and Grant 2001) and other related behaviours (Kramer and McLaughlin 2001). However, while some studies have touched on the underlying perceptual and energetic bases for intermittent locomotion (Gleeson and Hancock 2001; McLaughlin and Grant 2001; Hancock and Gleeson 2005), to our knowledge, no study has yet examined this form of locomotion in the context of animal 
'personality' (sensu Réale et al. 2007) and what role personality may play in its underlying mechanistic basis.

Animal personality, or temperament, has broadly been defined as consistency in an individual's behavioural responses over time and/or situations (Réale et al. 2007). One axis of behavioural variation, which has potentially important implications for behavioural ecology and particularly the study of intermittent locomotion, is that of boldness. Boldness is a personality trait that is characterized by individual differences in willingness to explore and take risks in a variety of behavioural contexts (Wilson et al. 1994; Ward et al. 2004; Webster et al. 2007; Wilson and Godin 2009). Although variation in both intermittent locomotion and boldness has been separately observed in some species [e.g. Eastern chipmunk, Tamias striatus (Trouilloud et al. 2004; Martin and Réale 2008); brook charr, Salvelinus fontinalis (McLaughlin and Grant 2001; Farwell and McLaughlin 2009)], surprisingly no attempt has been made to characterize and understand the relationship between these two behavioural traits at the individual level. Boldness, like intermittent locomotion, is associated with individual differences in general activity (Wilson and McLaughlin 2007), exploration and antipredator behaviour (Brown et al. 2005; Wilson and Godin 2009), mating (Godin and Dugatkin 1996) and dispersal (Fraser et al. 2001). Furthermore, several studies have posited that boldness is linked to fitness-related traits (Smith and Blumstein 2008), is heritable (Dingemanse et al. 2004; Sinn et al. 2006), and subject to selection (Réale and Festa-Bianchet 2003; van Oers et al. 2004). Thus, should boldness also be associated with intermittent locomotion, studying this relationship may offer further insights into the ecological and evolutionary 
implications of personality traits and animal movement, as well as their underlying mechanistic bases.

Here, we investigated the potential relationship between boldness behaviour and aspects of intermittent locomotion in individual field-caught juvenile bluegill sunfish (Lepomis macrochirus). We characterized this relationship by exposing individual sunfish to an open arena test in the laboratory and quantifying their willingness to take risks and explore a novel environment. Our primary focus in this study was to ascertain how individuals moved while exploring and assessing potential risk. Sunfish are ideal candidates for studies of intermittent locomotion and boldness for a number of reasons. First, they exhibit two kinematically distinct modes of swimming: labriform swimming at lower speeds and undulatory swimming at higher speeds (Jones et al. 2007; Kendall et al. 2007). Labriform locomotion occurs when both lift and thrust are generated through the use of the pectoral fins without flexing the long body axis. In contrast, undulatory locomotion involves primarily the caudal fin and undulations of the long body axis to generate thrust. Second, sunfish exhibit distinct morphological and behavioural variation in relation to individual differences in habitat and resource use (Robinson et al. 1993; Skúlason and Smith 1995) and are commonly used to study fish locomotion (e.g. Jones et al. 2007; Kendall et al. 2007) and personality (e.g. Wilson et al. 1993; Coleman and Wilson 1998; Wilson and Godin 2009). Lastly, our recent mark-recapture study (Wilson and Godin 2009; Chapter 2 above), on the same individuals as those used in the current study, demonstrated that aspects of boldness behaviour are consistent in the field and the laboratory for individual juvenile sunfish and can be repeatable for at least 6-8 weeks in the wild. 


\section{METHODS}

\section{Field collection of subjects}

Between 25 June and 7 August 2007, we collected 60 juvenile (Yr. 1+) bluegill sunfish (fork length: $6.2-8.3 \mathrm{~cm}$; weight: $3.55-9.17 \mathrm{~g}$ ) from the littoral zone of a small bay in Patterson Lake, Ontario, Canada $\left(44^{\circ} 34^{\prime} \mathrm{N}, 76^{\circ} 21^{\prime} \mathrm{W}\right)$ using a $10-\mathrm{m}$ beach seine. Captured individuals were placed in a cooler containing lake water (mean temperature $=23.1^{\circ} \mathrm{C}$ ) and transported to our laboratory at Carleton University (transit time $\sim 1.5 \mathrm{~h}$ ). Ten individuals were captured during each collection period, once per week. Patterson Lake is a small north-temperate lake $\left(<3 \mathrm{~km}^{2}\right.$ total area); it is a high-predation environment, containing many piscivorous fishes (e.g. smallmouth and largemouth bass, northern pike, pickerel) and birds (e.g. belted kingfisher, northern loon, great blue heron).

\section{Experimental holding conditions and general experimental apparatus}

Upon arrival at the laboratory, each wild-caught fish was placed singly into the 'refuge' area of one of 10 identical glass aquaria ( $82 \mathrm{~L}, 92 \times 30 \times 30 \mathrm{~cm}$, Figure 3-1), similar to those used by Wilson and Godin (2009). Each aquarium contained aerated and filtered dechlorinated tap water maintained at $23 \pm 1{ }^{\circ} \mathrm{C}$ and a gravel substratum. All aquaria were exposed to overhead fluorescent lighting on a $12 \mathrm{~h}$ light : $12 \mathrm{~h}$ dark cycle. Each aquarium was 'divided' into thirds both horizontally and vertically with lines drawn on the front and back walls. In doing so, 9 distinct zones were delineated to facilitate the recording of fish water-column use. The 'refuge' area was located at one end of the 
aquarium, contained a corner filter and a plastic aquarium plant for cover, and was separated from the remainder of the aquarium by an opaque white plastic partition equipped with a sliding door $(9 \mathrm{~cm} \times 12 \mathrm{~cm}$; Figure $3-1)$. This gated partition was located $25 \mathrm{~cm}$ from the left side of the aquarium. All aquaria were covered externally with tan cardboard at both ends and the back wall to prevent interaction between subjects in other aquaria. To avoid any confounding effects associated with olfactory cues from conspecifics, the water in all experimental aquaria was replaced with new water between trials.

All aquaria were placed behind an observation blind to minimize external disturbance and to facilitate the recording of behavioural trials. All trials were recorded using a Digital 8 video camera (Sony DCR-TRV280), and fish behaviours were quantified from the tapes using the behavioural analysis software JWatcher (v1.0) (Blumstein and Daniel 2007).

\section{Measures of boldness (risk-taking) behaviour in a novel environment}

Approximately $24 \mathrm{~h}$ after arrival in the laboratory, individual sunfish were tested in their respective aquarium for their level of boldness in a novel environment (Figure 3-1). At the onset of each behavioural trial, the sliding door of the test aquarium was lifted manually via a remote pulley system operated from behind the blind. The test fish was then able to enter and swim freely throughout the remaining open area of the aquarium (hereafter the 'arena'). Each fish was given a maximum of $60 \mathrm{~min}$ to exit the refuge area and explore the arena. Upon exiting the refuge, several measures of individual boldness were quantified for $10 \mathrm{~min}$. These boldness measures were latency to emerge from the 
refuge area, general activity in the open arena, and total time spent in the upper third of the water column of the arena. Increased general activity, emergence from a refuge, and time spent near the water surface are behaviours that are inherently risky and which may increase the risk of predation in animals, particularly fishes (e.g. Lima and Dill 1990;

Godin 1997; Sih 1997; Wilson and McLaughlin 2007). Here, we defined latency to exit the refuge as the total time elapsed from the moment the gated door was raised to the focal fish completely exiting the refuge area during the $60 \mathrm{~min}$ pre-trial period. Those individuals that did not exit within $60 \mathrm{~min}(n=13)$ were excluded from any further behavioural trials and analyses. Because we were primarily interested in measures of risk-taking, behaviours were only quantified while the focal fish was in the presumably more risky open arena of the aquarium, not in the refuge. We quantified general activity as the total time the focal fish spent active (versus being sedentary) outside the refuge in the open arena. Moreover, activity in the open arena was categorized by swimming mode and intermittent locomotion, as outlined below.

\section{Measures of intermittent locomotion}

The locomotor activity of individual test fish was quantified from trial videotapes using JWatcher (v1.0) as follows. For each individual fish, the number and duration of stationary pauses during the 10-min trial were recorded. A pause was defined as an interruption of self-propelled motion, wherein the fish was either at rest on the substrate or maintaining a stationary position in the water column through use of only the pectoral and anal fins. Activity was quantified as time spent moving 'fast' $(>3 \mathrm{~cm} / \mathrm{s})$ and time spent moving 'slow' $(<3 \mathrm{~cm} / \mathrm{s})$. Based on our frame-by-frame analysis of the video 
tapes, we note that this threshold swimming speed $(3 \mathrm{~cm} / \mathrm{s})$ corresponds to the transition between labriform and undulatory modes of locomotion in juvenile bluegill sunfish in our experimental aquaria. Fast movement, or undulatory swimming, typically incorporated the caudal fin as the main means of propulsion, with comparatively minimal contribution to forward motion from other fins. In contrast, slow movement, or labriform swimming, generally involved a gentle sculling movement of the pectoral fins, with little input from the caudal fin. For all forms of activity, the number of bouts of activity, the duration of each bout, and the mean duration of bouts overall were quantified. Any movement of the body forward or backward, irrespective of fin use, was recorded as activity. It was not possible to consistently assess speeds of movement accurately beyond the conservative measure provided here and therefore we do not provide an estimate of distance travelled within the arena during trials. All videotape analyses were performed blind with respect to individual fish identity to minimize risk of observer bias.

At the end of an experimental trial, the standard length (SL) and wet weight (W) of the focal individual were recorded and its body condition calculated as [W $(\mathrm{g}) / \mathrm{SL}^{3}$ (cm)] x 100 (Bolger and Connolly 1989).

\section{Data analyses}

First, comparisons of individual boldness and locomotory traits within the context of exploration and risk-taking in a novel environment were made using the non-parametric Spearman's rank correlation test. Second, to test for any relationships between boldness and modes of intermittent locomotion more directly, boldness and locomotory traits (fast/undulatory and slow/labriform) were collapsed into first principal component scores 
for each axis using Principal Components Analysis (PCA) (Table 3-1). Correlations between PC1 scores for boldness and locomotion traits were then calculated using the Spearman's rank correlation test as above. To minimize Type I errors in our statistical analyses, the alpha level $(\alpha=0.05)$ was adjusted to be more conservative using the sequential Bonferroni correction (Rice 1989).

\section{RESULTS}

\section{Correlations across behavioural and locomotion traits}

These correlations are pairwise Spearman's rank correlations ( $n=47$ fish). In general, individual fish that spent more time moving fast $(>3 \mathrm{~cm} / \mathrm{s})$ exhibited shorter latencies to emerge from the refuge $\left(r_{s}=-0.77, P<0.0001\right.$; Figure $\left.3-2 a\right)$, shorter sedentary pauses $\left(r_{s}\right.$ $=-0.80, P<0.0001 ;$ Figure $3-2 \mathrm{~b})$, and longer bouts of activity $\left(r_{s}=0.72, P<0.0001\right)$ than individuals that moved more slowly $(<3 \mathrm{~cm} / \mathrm{s})$. Faster individuals also spent more time near the water surface (upper third of water column; $r_{s}=0.44, P=0.002$; Figure 32c).

In contrast, those individuals that spent more time moving slowly tended to prefer the lowest third $\left(r_{s}=0.42, P=0.0032\right)$ over the upper third of the water column $\left(r_{s}=-\right.$ $0.21, P=0.1617$; Figure $3-2 \mathrm{c})$ and, as expected, took longer to emerge from refuge $\left(r_{s}=\right.$ $0.75, P<0.0001 ;$ Figure 3-2a) and had longer sedentary pauses $\left(r_{s}=0.77, P<0.0001\right.$;

Figure 3-2b) whilst in the open arena. Body size (standard length, weight) and body condition were not significantly correlated with any boldness or locomotory traits (all $P>$ 0.05, Bonferroni corrected). 


\section{Principal components analysis of boldness and intermittent locomotion traits}

The PC1 scores for boldness and intermittent locomotion behaviour of sunfish in a novel environment (Table 3-1) were significantly correlated with each other. More specifically, boldness was strongly positively correlated with undulatory or fast swimming $\left(r_{s}=0.58\right.$, $P<0.0001$; Figure 3-3a), but conversely negatively correlated with labriform or slow swimming $\left(r_{s}=-0.46, P=0.001\right.$; Figure $\left.3-3 b\right)$. Neither boldness nor locomotion principal component scores were correlated with body size or body condition (all $P>$ 0.05 , Bonferroni corrected).

\section{DISCUSSION}

We found that juvenile bluegill sunfish exhibited inter-individual differences in modes of intermittent locomotion, which in turn were correlated with differences in their boldness (risk-taking) behaviour. While bold and timid individuals may have utilized both undulatory and labriform modes of locomotion, the amount of time spent in each mode and their associated pauses differed between behavioural types. Generally, bolder individuals spent more time moving fast (undulatory swimming), for longer durations and with shorter pauses than more timid, slower-moving (labriform swimming) individuals. Correspondingly, as revealed by our PCA analysis, bolder individuals had higher intermittent locomotion scores when moving fast and conversely lower intermittent locomotion scores when moving slowly, and vice versa for timid individuals. To our knowledge, the current study is the first to have characterized the relationship between intermittent locomotion and boldness in any species. 
Our findings are novel and important to behavioural ecologists, as both boldness and intermittent locomotion are associated with foraging, mating, exploration, dispersal, and antipredator behaviours in animals (Kramer and McLaughlin 2001; Sih et al. 2004; Dingemanse and Réale 2005; Réale et al. 2007). Indeed, in a related study (Wilson and Godin 2009; Chapter 2) on the same individual sunfish that were used in the current study, we found that boldness variables (i.e. refuge emergence and distance from refuge) were highly correlated with individual differences in exploration and general activity in multiple behavioural contexts, forming a behavioural syndrome. Further, we demonstrated that these inter-individual differences in behaviour were both consistent and repeatable in the field as part of a mark-recapture study over a period of two months (Wilson and Godin 2009; Chapter 2).

To date, intermittent locomotion has typically been considered only in the contexts of foraging and vigilance or anti-predator behaviour (e.g. McAdam and Kramer 1998; Kramer and McLaughlin 2001; Trouilloud et al. 2004). We suggest that this type of locomotion may also play an important role in general exploration and risk assessment in novel environments and/or situations. Intermittent locomotion may therefore offer a novel paradigm to evaluate animal personality (i.e. boldness) and, more specifically, to predict behaviours of important ecological interest, such as dispersal tendency and invasiveness. For example, previous studies have suggested that bolder individuals are not just more active, but also tend to disperse further, than more timid individuals (e.g. Fraser et al. 2001; Dingemanse et al. 2003). Since boldness is both heritable and subject to selection (e.g. Dingemanse et al. 2002; Réale and Festa-Bianchet 2003; Sinn et al. 2006), the relationship between boldness and intermittent locomotion, described herein, 
may offer new insight into the origin of these individual-level differences in behaviour and how they are maintained in natural populations.

Previous studies on intermittent locomotion have focused primarily on energetic explanations for individual-level differences or on morphological correlates of such movement (e.g. Brana 2003; Trouilloud et al. 2004). Comparatively few studies have considered possible perceptual or behavioural (functional) explanations for intermittent locomotion. Kramer and McLaughlin (2001) suggested that self motion can interfere with visual perception or visual acuity. Though sensory systems are inherently designed to reduce such interference, animals commonly exhibit bursts of movement interspersed with short stationary pauses while foraging (McLaughlin and Grant 2001; Trouilloud et al. 2004) or when exhibiting antipredator behaviour (e.g. predator inspection; Dugatkin and Godin 1992). It is therefore possible that such pauses facilitate the visual assessment of environmental cues within the animal's visual field (cf. Kramer and McLaughlin 2001). However, this hypothesis remains untested and does not exclude metabolic or energetic factors determining such inter-individual differences in locomotion.

Fishes exhibit considerable variation in swimming speeds (i.e. undulatory/labriform swimming), which, in addition to energetic considerations (e.g. muscle type ratios; metabolic rate), may reflect adaptive processes allowing for more effective sensory processing and risk assessment. For example, the likelihood of detecting a potential threat (e.g. predator) or food item generally increases with the duration of visual scanning-type behaviour (though detection is also influenced by head/eye movement and interscan intervals; Hart and Lendrem 1984; Bednekoff and Lima 2002) in animals. However, such a relationship may be constrained by the higher 
levels of activity (Barros et al. 2004) commonly exhibited by bold individuals (Kortet and Hedrick 2007; Webster et al. 2007; Wilson and Godin 2009), irrespective of locomotory pause frequency and duration.

In the current study, we found no differences in body size or body condition between timid and bold sunfish, suggesting that these fish had similar energetic states. Recent work on juvenile bluegill sunfish (T.R. Binder and A.D.M. Wilson, unpublished data) has revealed that individual differences in boldness (as measured by latency to exit a refuge) do not appear to be related to underlying individual differences in standard metabolic rate. The above proposition is also supported by similar findings in other fishes testing for individual differences in body size or metabolic rate relative to boldness or locomotory behaviour (e.g. Sundström et al. 2004; Farwell and McLaughlin 2009). Further, since inter-individual variation in boldness can be influenced by experience (Wilson et al. 1993; Frost et al. 2007; Martin and Réale 2008), it is likely that individual differences in perceptual ability and experience represent important correlates of boldness and intermittent locomotion, irrespective of the possible involvement of energetic factors.

Nonetheless, the energetic basis of individual variation in boldness, as with intermittent locomotion, and its relationship to fitness correlates is a subject of current interest (Réale et al. 2007; Smith and Blumstein 2008; Careau et al. 2008). The relationship between the shy-bold axis of personality and intermittent locomotion, which we characterized here for the first time using the bluegill sunfish, offers an opportunity to investigate such inter-individual variation in behaviour from a physiological perspective, incorporating both genetic and condition-dependent factors (e.g. red: white muscle index, liver glycogen, fat stores). Furthermore, much as boldness varies between behavioural 
contexts (e.g. Coleman and Wilson 1998; Wilson and Stevens 2005; Webster et al. 2007), so does the behaviour of individuals exhibiting intermittent locomotion (current study). Patterns of intermittent movement vary with changes in the type of behaviour exhibited by individual animals (e.g. changes in habitat, foraging, vigilance; Kramer and McLaughlin 2001). However, the inherent trade-offs associated with changes in exploratory, foraging and anti-predator behaviours remain largely unknown in the context of intermittent locomotion and represent a subject for further investigation. 


\section{ACKNOWLEDGEMENTS}

We thank R. Bell for permission to access Patterson Lake and the Ministry of Natural Resources of Ontario for the necessary fish collection permit. This study was approved by the institutional Animal Care Committee at Carleton University (protocol B06-7) and thus adheres to the guidelines of the Canadian Council on Animal Care and the laws of

Canada. Funding: The Natural Sciences and Engineering Research Council of Canada

(Discovery research grant to J.-G.J.G. and Post-graduate scholarship to A.D.M.W.). 
Table 3-1: PCA loadings of behavioral and locomotory variables used to generate a principal component score (PC1) to assess correlations between boldness and modes of intermittent locomotion in individual juvenile bluegill sunfish.

\begin{tabular}{llcl}
\hline Trait & Behaviours within each context & $\begin{array}{c}\text { Loadings for } \\
\text { PC1 }\end{array}$ & $\begin{array}{c}\% \\
\text { variation } \\
\text { explained }\end{array}$ \\
\hline Boldness & Latency to emerge from refuge & -0.7071 & 72.7 \\
& Time spent near water surface & 0.7071 & \\
\hline Intermittent locomotion & Time spent swimming fast & 0.7071 & 77.6 \\
(undulatory/fast swimming) & Mean duration of sedentary pause & -0.7071 & \\
\hline Intermittent locomotion & Time spent swimming slow & 0.7071 & 64.4 \\
(labriform/slow swimming) & Mean duration of sedentary pause & 0.7071 & \\
& & & \\
\hline
\end{tabular}




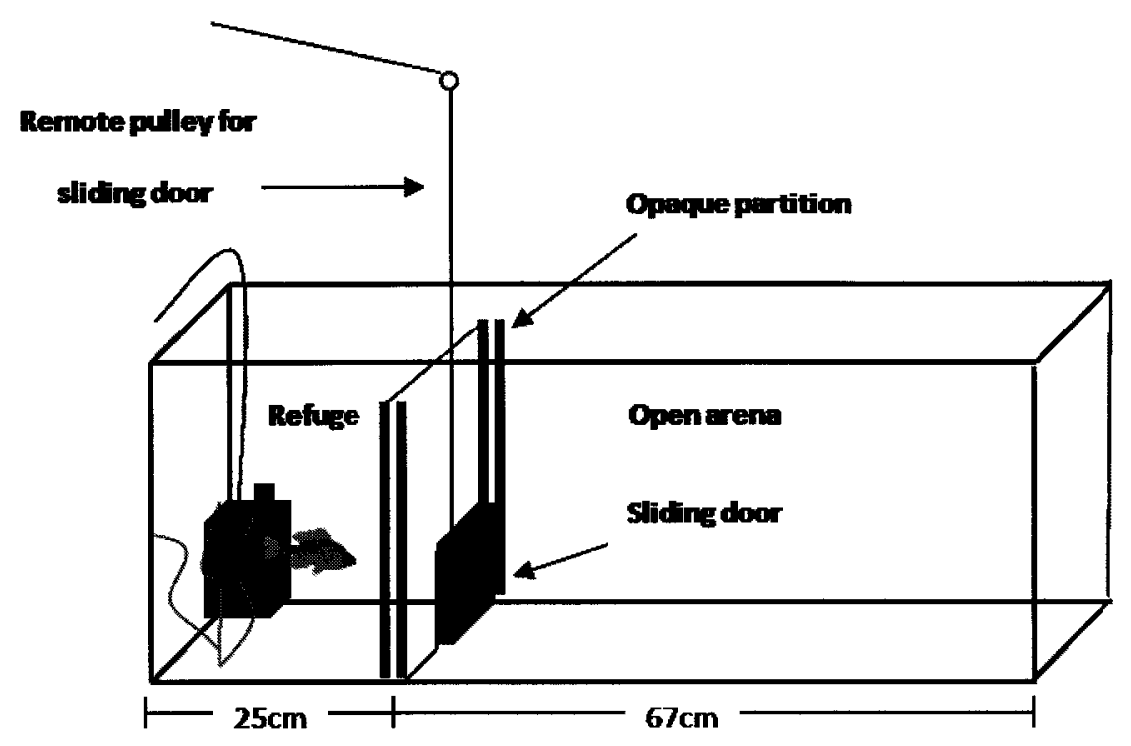

Figure 3-1: Schematic representation of the experimental aquarium used to quantify

boldness and intermittent locomotion behaviours of individual juvenile bluegill sunfish in the laboratory. 


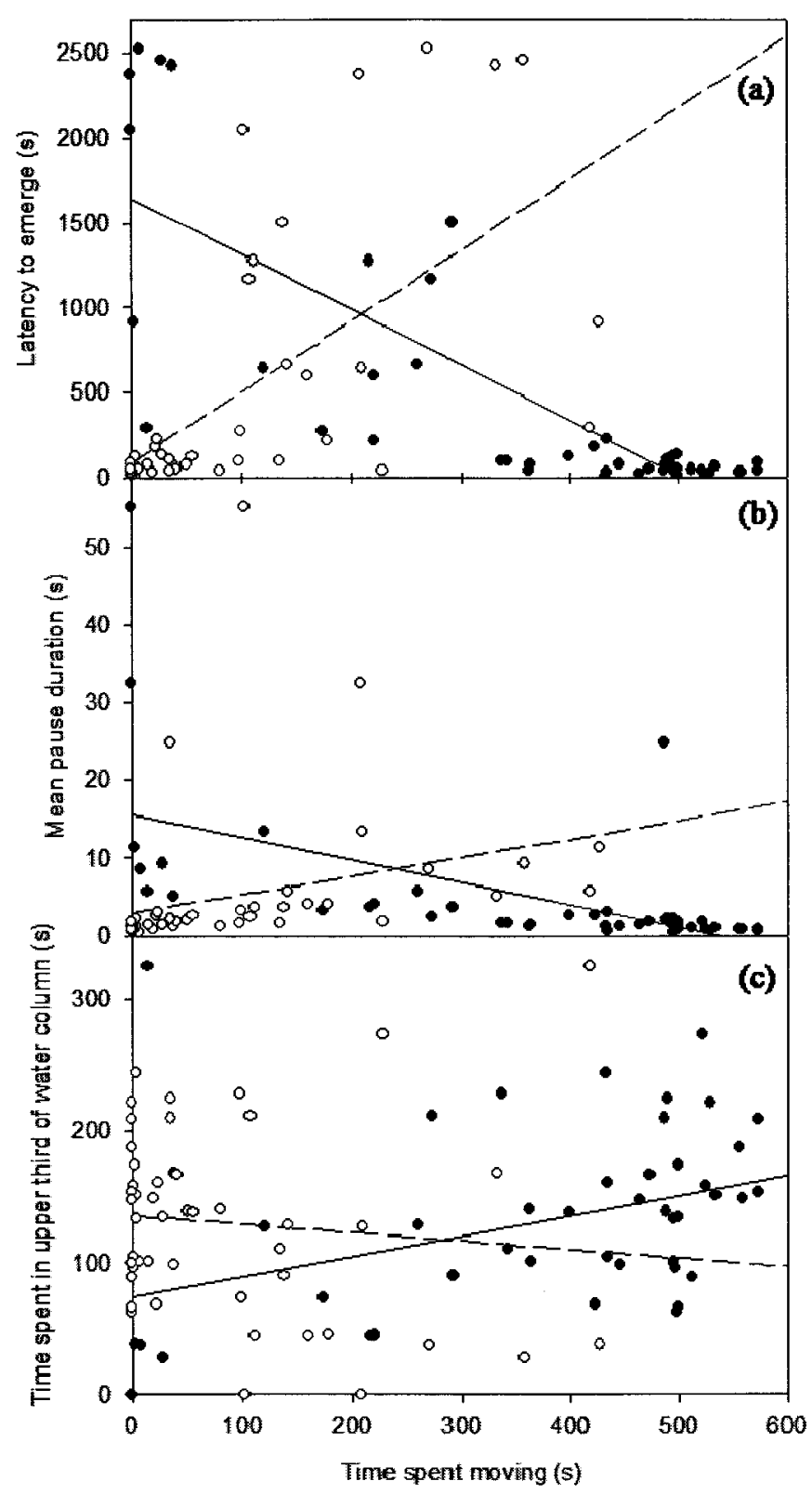

Figure 3-2: Individual differences in latency to emergence from a refuge (a), sedentary pause duration (b), and time spent near the water surface (c) in relation to undulatory (fast) and labriform (slow) swimming behaviour in juvenile bluegill sunfish. Each data point represents an individual $(n=47)$. Open circles $(0)$ and dashed lines represent values for time spent moving slowly $(<3 \mathrm{~cm} / \mathrm{s})$. Closed circles $(\bullet)$ and solid lines represent time spent moving fast $(>3 \mathrm{~cm} / \mathrm{s}$ ). Each line represents a line-of-best-fit through the relevant data obtained from a least-square regression. 


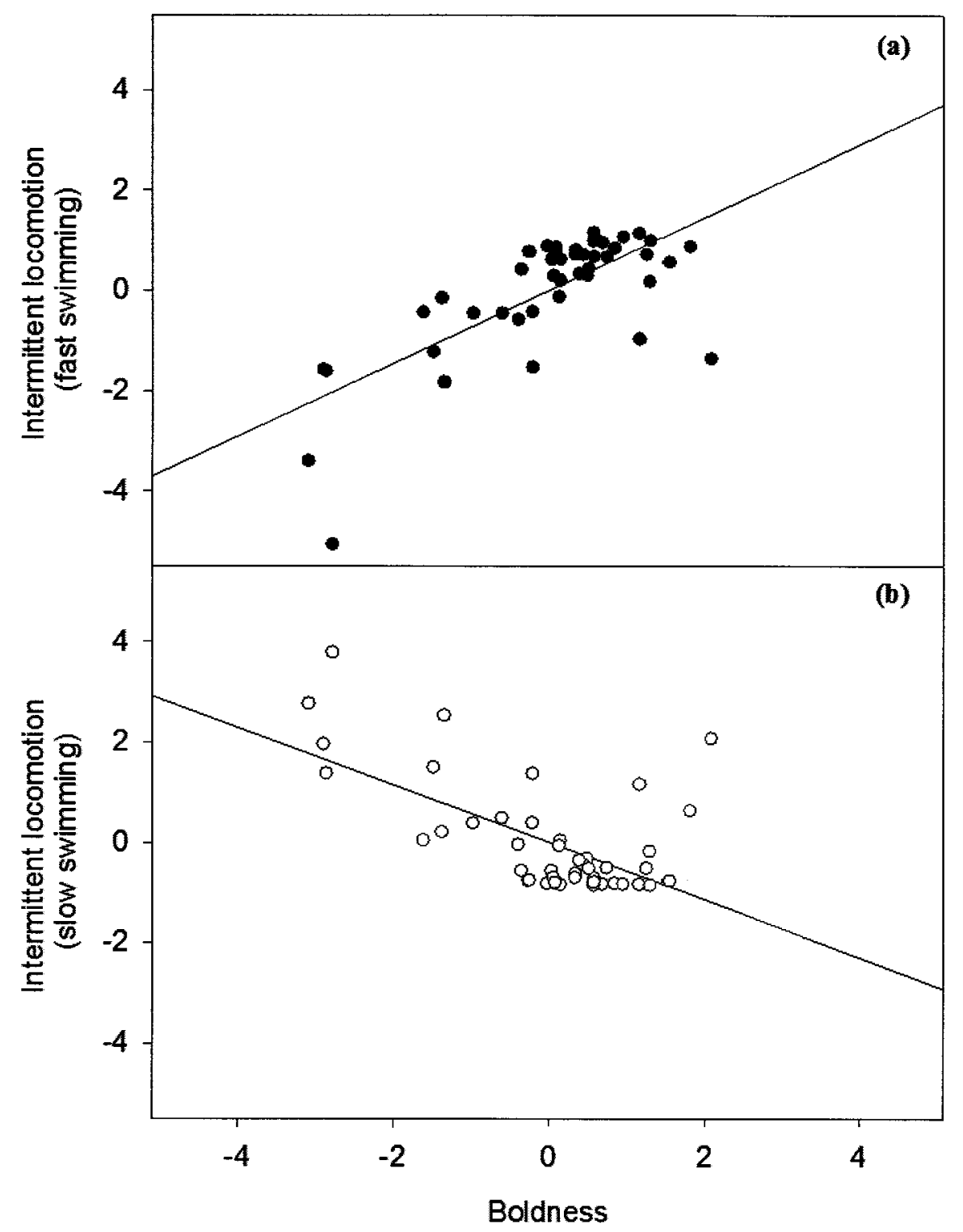

Figure 3-3: Individual differences in modes of intermittent locomotion (undulatory/fast [a]; labriform/slow [b]) relative to boldness in juvenile bluegill sunfish $(n=47)$ in a novel environment. Each data point represents an individual's principal component (PC1) score for each trait shown. For each panel, the line shown represents a line-of-best-fit through the data obtained from a least-square regression. 


\section{CHAPTER 4}

\section{CAPTURE TECHNIQUE AND FISH PERSONALITY: RECREATIONAL ANGLING TARGETS TIMID BLUEGILL SUNFISH, LEPOMIS MACROCHIRUS ${ }^{*}$}

* Under review, Behavioral Ecology

Wilson ADM, Binder TR, McGrath KP, Cooke, SJ, Godin, J-GJ. Capture technique and fish personality: recreational angling targets timid bluegill sunfish, Lepomis macrochirus. Submitted to Behav. Ecol. 


\begin{abstract}
Anthropogenic factors may influence the evolution of both morphological and behavioural traits in natural animal populations. For example, size-selective harvesting associated with commercial and recreational fishing practices has been shown to alter life history traits through a phenomenon known as fishing-induced evolution. However, lifehistory changes associated with fishing practices may be a result of selection pathways targeting life-history traits directly (e.g. decreased intrinsic growth) or indirectly through correlations with behavioural traits (e.g. personality). Here, we report on the relationship between individual differences in behaviour and capture technique (beach seining versus angling) in wild-caught juvenile bluegill sunfish (Lepomis macrochirus). Both seined and angled sunfish were individually tested under standardized laboratory conditions for their boldness, water column use and general activity. Following testing, individuals caught initially via seining were tagged and transferred to a large outdoor pool for further testing using a modified angling protocol. Observed inter-individual differences in boldness were strongly correlated with method of capture in the wild. Angled fish were more timid and had fewer ectoparasites than seined individuals. However, this relationship did not carry over to our experiment in the outdoor pool, where bolder individuals were more likely to be angled in open water away from refuges than more timid individuals based on their previously assessed boldness scores. Our study is both novel and important as it describes the relationship between capture technique and boldness in a natural population and also underscores the potential risk of sampling biases associated with method of animal capture for behavioural researchers.
\end{abstract}




\section{INTRODUCTION}

Animal 'personality' is commonly defined as individual differences in behaviour that are consistent across time and/or situations, and can incorporate a broad range of behavioural axes (e.g. boldness, sociability, aggressiveness) (Réale et al. 2007). Interest in the underlying proximate and evolutionary bases of animal personality has been steadily increasing in recent years (e.g. van Oers et al. 2008; Réale et al. 2009; Wilson and Godin, 2009), particularly with reference to the mechanisms maintaining individual-level differences in behaviour, their origin, and heritability (Réale et al. 2007; Smith and Blumstein, 2008). While some studies have examined a variety of natural selection pressures shaping such behavioural traits (e.g. predation, environmental heterogeneity; (e.g. predation, environmental heterogeneity; see Dingemanse and Réale, 2005), other recent studies have highlighted the potential impact of anthropogenic factors on the evolution of both morphological and behavioural traits (McDougall et al. 2006; Allendorf and Hard, 2009). For example, size-selective harvesting associated with commercial and recreational fishing practices has been shown to alter life history traits (e.g. earlier sexual maturation, reduced adult body size) through a phenomenon known as fishing-induced evolution (FIE) (Heino and Godo, 2002; Kuparinen and Merila, 2007; Uusi-Heikkila et al. 2008). However, as postulated by Uusi-Heikkila et al. (2008), life-history changes associated with fishing practices may be a result of selection pathways targeting lifehistory traits directly (e.g. decreased intrinsic growth) or indirectly through correlations with behavioural traits. Indeed, the efficacy of certain animal capture methods does appear to be dependent more on the behaviour of the targeted animals than on their morphological traits per se (Biro and Post, 2008; Uusi-Heikkila et al. 2008). For example, 
individuals that are more active, exploratory and likely to take risks may disproportionately be susceptible to capture and, as a consequence, this may result in a variety of deleterious side-effects (e.g. rapid depletion of certain behavioural phenotypes and genotypes from a population) that may have important implications for species management and conservation (McDougall et al. 2006; Biro and Post, 2008; Allendorf and Hard, 2009). In addition, this phenomenon may have further implications for the manner by which animals are collected for behavioural research, as different capture methods may unintentionally target specific behavioural phenotypes (e.g. personality types), resulting in potential sampling biases (Biro and Dingemanse, 2009).

One axis of personality that has particular relevance to behavioural biologists is that of boldness. Boldness may be defined as the tendency of an individual to take risks (Wilson et al. 1994). The ecological and evolutionary importance of boldness is evident in both its taxonomic expression and the broad range of other ecologically-relevant behaviours with which it is associated. Individual differences along the boldness axis have been documented in a variety of taxa, including fishes (Ward et al. 2004; Webster et al. 2009; Wilson and Godin, 2009), birds (Carere and van Oers, 2004; van Oers et al. 2004; Both et al. 2005), mammals (Réale and Festa-Bianchet, 2003; Réale et al. 2009), reptiles (Lopez et al. 2005), cephalopods (Sinn et al. 2006, 2008), and insects (Kortet et al. 2007; Wilson et al. 2010a). Boldness is also associated with numerous ecologicallyrelevant behaviours such as general activity and space use (Wilson and McLaughlin, 2007), intermittent locomotion (Wilson and Godin, 2010; Chapter 3 above), sociability (Ward et al. 2004; van Oers et al. 2005; Webster et al. 2007), antipredator behaviour (Wilson and Godin, 2009; Chapter 2 above; Jones and Godin, 2010), invasiveness 
(Rehage and Sih, 2004), dispersal (Fraser et al. 2001), mate selection (Godin and Dugatkin, 1996), foraging (Farwell and McLaughlin, 2009), and exploration (Wilson and Stevens, 2005; Wilson and Godin, 2009). Furthermore, boldness is also correlated with fitness-related traits (Smith and Blumstein, 2008; Wilson et al. 2010b), is heritable (Dingemanse et al. 2002; Brown et al. 2007), and subject to natural (Réale and FestaBianchet, 2003) and 'unnatural' (Biro and Post, 2008) selection.

Here, we used juvenile bluegill sunfish (Lepomis macrochirus) to ascertain whether recreational angling targets timid or bold individuals differentially using both field and laboratory techniques and, additionally, to compare different capture practices for the potential presence of a sampling bias. Sunfish are particularly amenable to this type of study as they are easily captured using a variety of methods (e.g. line angling, seine), are abundant in many freshwater systems across southern Ontario, Canada (Scott and Crossman, 1998), and are known to exhibit individual-level differences in boldness (Wilson et al. 1993; Wilson and Godin, 2009, 2010; Chapters 2 and 3). Furthermore, bluegill sunfish exhibit dramatic changes in morphological and behavioural life-history traits in response to harvesting exploitation by recreational angling (Coble, 1988; Jennings et al. 1997; Beard and Essington, 2000). Since bluegill sunfish are a popular sport fish across North America (Spotte 2007), any potential capture biases associated with individual differences in personality may have significant evolutionary and ecological consequences for the affected populations, as well as the quality of the fishery (Beard and Essington, 2000; Biro and Post, 2008), and may influence management strategies (Ashley et al. 2003). 
Firstly, to quantitatively assess the relationship between angling and personality in bluegill sunfish, we captured juveniles from a large north-temperate lake (using both angling and seining techniques) and exposed them to a standardized behavioural test quantifying refuge emergence time (a measure of boldness), general activity, and water column use following the methodology of Wilson and Godin (2009). Should recreational angling target either bold or timid individuals differentially, we predicted that fish captured via angling in the wild would be bolder on average than those individuals caught haphazardly with a beach seine. Secondly, to characterize the relationship between boldness and susceptibilty to angling, all seined individuals were placed in a large outdoor pool (following behavioural testing), wherein we attempted to recapture these individuals using a standardised angling protocol. We predicted that individuals that were scored as being bolder during laboratory trials would be more likely to be caught via angling than more timid individuals, as per our previous prediction for field-captured fish. In addition, since bolder individuals tend to spend more time in riskier areas of the water column (e.g. near surface, open water; Wilson and Godin, 2009; Chapter 2), we also predicted that, on average, bolder individuals would be more likely to be caught in the open portion of the pool, than near a provided refuge, when compared to more timid fish. Understanding the relationship between recreational angling and fish boldness should contribute to our understanding of the potential ecological and evolutionary consequences of such harvest practices on natural populations, and also offer further insight into the potential for sampling biases in behavioural research based on method of animal capture. 


\section{METHODS}

\section{Field collection}

Between June 10 and July 20, 2009, we used two different collection techniques to capture 230 juvenile (Yr. 1 +) bluegill sunfish (Total Length [TL]: 98 - 148 mm; weight: $14.1-58.5 \mathrm{~g}$ ) from the area within and around several small bays (littoral zone) in Lake Opinicon, Ontario, Canada $\left(44^{\circ} 34^{\prime} \mathrm{N}, 76^{\circ} 21^{\prime} \mathrm{W}\right)$. One of these collection techniques involved the use of a $20-\mathrm{m}$ beach seine ( $5 \mathrm{~mm}$ mesh size) to capture a subset of the above individuals $(N=120,98$ - $148 \mathrm{~mm}$ TL, 14.9 - $51.8 \mathrm{~g})$ from the littoral zone of several shallow bays. Seining involved making numerous attempts of short duration (2-3 min for net movement, 5-7 min for fish collection from the net). Such brief seining attempts were used to minimize physical injury and stress among captured fish. Following each attempt, all captured fish of the appropriate size were immediately placed inside a cooler with fresh lake water (see below).

The other collection technique involved a standardized 10-s angling protocol by which we captured the remaining portion of individuals used in the study $(N=110,99$ $148 \mathrm{~mm}$ TL, 14.1 - $58.5 \mathrm{~g}$ ) over several collection trips. This short angling duration (i.e., $10 \mathrm{~s}$ ) is common for sunfish (Cooke et al. 2003). These individuals were captured from the same bays as those sampled with the beach seine. In accordance with our angling protocol, only small, barbless hooks baited with a 1-cm piece of earthworm (Lumbricus sp.) were used to capture fish. Moreover, individuals had to be caught, have the hook removed, and be placed inside a cooler within 10-s to be included in our study. Any individuals caught that did not follow this protocol, or individuals that were injured (e.g., any evidence of bleeding) as a result of angling practices, were excluded from further 
study. This protocol insured that any physical trauma and stress associated with capture would be minimized.

Following capture (via both methods), the fish were transported in coolers containing lake water (mean temperature $=23.4^{\circ} \mathrm{C}$ ) to our laboratory facilities at the Queen's University Biological Station, located on the shoreline of Lake Opinicon (boat transit time $\sim 30 \mathrm{~min}$ ). Between 16 and 20 (mean $=18$ ) individuals were captured during each collection period, twice per week. Seining and angling did not occur on the same day.

\section{Experimental holding conditions and experimental apparatus}

On arrival at the laboratory, fish captured either by angling or seining were placed in one of two large holding tanks $(1401,61 \times 67 \times 42 \mathrm{~cm}, \mathrm{~L} \times \mathrm{W} \times \mathrm{H})$. Each holding tank was aerated and continually provided with fresh water from the lake via a flow-through system. The tanks were exposed to ambient sunlight through several windows in the laboratory. All fish were held overnight prior to experimental testing the following day.

Since angled and seined fish were respectively caught on consecutive days (not concurrently), the particular holding tank assigned to each group was randomly assigned each week to reduce the likelihood of any holding tank bias. Each holding tank was equipped with six plastic aquarium plants of various sizes (height range $=20-60 \mathrm{~cm}$ ) to provide cover and minimize agonistic interactions between fish. The density of sunfish (< 20 on average) held in each holding tank falls within the range of densities observed in free-ranging juvenile bluegill sunfish in the shallow littoral zone of Lake Opinicon and similar nearby lakes in eastern Ontario (A.D.M. Wilson, pers. obs.). 
During behavioural testing, each fish was placed in one of two identical glass aquaria $(821,92 \times 30 \times 30 \mathrm{~cm})$. Each aquarium contained lake water $\left(23 \pm 1{ }^{\circ} \mathrm{C}\right.$; replaced daily) and was exposed to overhead fluorescent lighting. Each aquarium was divided into thirds, both horizontally and vertically, with lines drawn on the front panel. In doing so, each aquarium was divided into nine distinct zones of equal size to facilitate the observation of fish water column use. A refuge area, consisting of three plastic aquarium plants for cover, was located on one side of the test aquarium and was separated from the remainder of the aquarium by an opaque white plastic partition equipped with a sliding door. This gated partition was located $25 \mathrm{~cm}$ from the left side of the aquarium (see Figure 2-1 above; cf.Wilson and Godin, 2009). Aquaria were covered externally with brown cardboard at both ends and the back wall to prevent external disturbance and interaction between subjects.

Both aquaria were placed behind an observation blind to minimize external disturbance and to facilitate the quantification of behavioural parameters. Behaviours were quantified using the behavioural analysis software JWatcher (v1.0) (Blumstein and Daniel, 2007).

\section{Behavioural experiments}

\section{Experiment 1: latency to exit a refuge and explore a novel environment}

Approximately $24 \mathrm{~h}$ after capture (by either seining or angling), each focal fish was gently hand-netted using an aquarium dip-net and placed within the refuge portion of an experimental aquarium (see above). The focal fish was then given $15 \mathrm{~min}$ to acclimatize to the refuge prior to the onset of behaviour testing. Following this period, the sliding 
door of the test aquarium was lifted manually via a remote pulley system operated from behind the blind, thereby allowing the focal fish to exit the refuge area and swim freely throughout the remainder of the aquarium (hereafter the "arena"). Each fish was given 20 min to exit the refuge and explore the arena. On exiting the refuge, several behavioural measures were quantified, including latency to exit the refuge, water column use, and total time spent active in the arena (see Day 1 protocol in Chapter 2 above; cf. Wilson and Godin, 2009). Because we were primarily interested in measures of risk-taking, only behaviours exhibited in the open arena (and not in the refuge) were quantified. Any individual that did not exit the refuge within the 20 -min pre-trial period was assigned a latency-to-exit score of $20 \mathrm{~min}$.

At the completion of behavioural trials, focal fish that were caught initially via seining were anesthetized using tricaine methanesulfonate (MS-222, 1:10 000 dilution) buffered with sodium bicarbonate, and individually tagged on their dorsum with visible elastomer implant tags (Northwest Marine Technology Inc.) following a standardized protocol (see Chapter 2 above: cf.Wilson and Godin, 2009). Following the tagging procedure, these fish were returned to their respective holding tank for recuperation until the onset of Experiment 2 the next day.

Only seined fish were held over for further behavioural tests; those individuals caught initially via angling were euthanized using a lethal dose of buffered MS-222. The standard length (SL) and wet weight (W) of these individuals were then recorded and their body condition calculated as $\left[\mathrm{W}(\mathrm{g}) / \mathrm{SL}^{3}(\mathrm{~cm})\right]$ x 100 (Bolger and Connolly, 1989). In addition, external parasites were counted (e.g. black spot, Uvulifer ambloplitis) as parasite load can affect behaviour in fishes (Barber et al. 2000) and has been shown to be 
associated with boldness in sunfishes (Wilson et al. 1993; Barber et al. 2000). Further, additional morphological measurements (e.g. total length, girth) were made, and any fish that was found to be reproductively mature $(N=2)$ was removed from the study.

\section{Experiment 2: boldness and susceptibility of seined fish to angling practices}

Approximately $16 \mathrm{~h}$ after being tested for boldness and tagged, a group of 15 fish (which had been previously seined from the lake) was transported from the indoor holding facilities to a large outdoor fibreglass pool tank (Figure 4-1) to assess the susceptibility of seined fish to a recreational angling protocol related to that used in the field. Following their addition to the outdoor tank, the test fish were given an additional $24 \mathrm{~h}$ to acclimatize to the tank before being subjected to a standardized angling protocol (see below). A total of eight such replicate groups of fish were similarly tested. This experiment aimed to characterize the relationship between fish boldness level and susceptibility to being captured by angling in a novel experimental environment.

The outdoor pool consisted of a large, grey, fibreglass circular tank $(366 \mathrm{~cm}$ diameter) and was exposed to natural sunlight. The oxygen level and temperature ( $23 \pm$ $1^{\circ} \mathrm{C}$ ) were consistently maintained via a continuous inflow of fresh water from the lake into the tank and surface outflow. Water level was maintained at $46 \mathrm{~cm}$ and the base of the tank was covered uniformly with natural rubble ( $~ 5 \mathrm{~cm}$ deep). Additionally, two refuges were added, each consisting of three concrete cinderblocks (each block $40 \times 20 \times$ $20 \mathrm{~cm}$ ) stacked in the shape of pyramid. The refuges faced each other from opposite sides of the tank $(\sim 200 \mathrm{~cm}$ apart) and were located $30 \mathrm{~cm}$ from the tank wall (Figure 41). 
The angling protocol used in this experiment mimicked the one used in the field as closely as possible. The observer (ADMW) was positioned centrally such that he was perpendicular to the direction of the two refuges and equidistant to each of them. During three distinct time periods (beginning at 09:00, 13:00 and 18:00 hrs) over the course of any experimental day, attempts were made to capture fish using a standardized angling technique. This technique was slightly modified from the angling protocol used in the field, but utilized the same equipment (i.e. hook, bait choice). Each fishing attempt lasted $20 \mathrm{~min}$ and involved alternating the dangling of the baited hook $30 \mathrm{~cm}$ from the front of each refuge for approximately $1 \mathrm{~min}$. Though the exact position of the hook varied each time, the distance from refuge remained relatively constant (see Figure 4-1).

When caught, individuals were identified, placed in coolers containing lake water, and brought back to the laboratory for measurement and determination of reproductive status (immature juvenile vs. mature adult, as above). After the last fishing period (18:20 hrs), any individuals that remained uncaught in the experimental pool were netted out and similarly processed. The time individuals were caught and their position of approach to the hook prior to being caught (i.e. open area vs. within refuge) were recorded for each fish. On the rare instance where a fish bit the hook but escaped capture $(N=3)$, an attempt was made to visually identify it based on its tag and where possible $(N=2)$ the fish was counted as being caught during that collection period.

\section{Experiment 3: stress assessment of the beach seining and angling protocols}

To ascertain the role, if any, capture method might have played in influencing the behaviour of focal fish, we measured plasma cortisol levels $(\mathrm{ng} / \mathrm{ml})$ in a number of seined 
$(N=21,110-142 \mathrm{~mm}$ TL$)$ and angled $(N=30 ; 100-141 \mathrm{~mm} \mathrm{TL})$ individuals collected using the exact same capture and holding protocols as described above. These individuals represent those fish from which sufficient amounts of plasma were collected within 30-s to allow the quantification of plasma cortisol. However, each holding tank did contain 20 individuals that were caught using either angling or seining techniques so as to mirror the conditions used for fish holding prior to Experiments 1 and 2.

Plasma cortisol is a known stress hormone in fishes and is commonly used as an indicator of overall stress during laboratory and field experimentation in fishes (Barton, 2002). Following a 24-h acclimatization period in laboratory holding tanks (as experienced by fish in Experiments 1 and 2), we collected blood samples from each of the fish to ascertain their level of stress, which we assume would be representative of that of fish just prior to behavioural testing in Experiments 1 and 2. Blood samples were collected using a $1 \mathrm{ml}$ heparinised syringe with a $25 \mathrm{~g}$ needle (Becton-Dickson, $\mathrm{NJ}$ ) via caudal puncture and were immediately stored in liquid nitrogen until they could be analyzed later in the laboratory. Each collected blood sample was centrifuged to yield plasma. Plasma cortisol was determined using a commercial kit (ImmunoChem Cortisol 125I RIA kit, MP Biomedicals, Orangeburg, NY) and a Cobra Auto-Gammer (HewlettPackard, Palo Alto, CA) following the methods outlined by Gamperl et al. (1994). Prior to plasma sample collection, all fish were anesthetised using buffered MS-222 (1:10 000 dilution).

It is important to note that the objective of this experiment was to ascertain whether our two methods of capture per se (and not our behavioural testing) are associated with different levels of stress in juvenile sunfish. Consequently, fish used in 
Experiments 1 and 2 were purposely not sampled for plasma cortisol following their initial holding period in the laboratory, as this procedure is invasive and would have confounded the subsequent behavioural trials that the fish were exposed to in both the laboratory aquaria (Experiment 1) and outdoor pool tank (Experiment 2).

\section{Data analyses}

First, comparisons of recorded individual behavioural measures across the two experimental groups (i.e. seined versus angled) were made using the nonparametric Mann-Whitney U test. These comparisons were made to assess behavioural differences between experimental groups based on capture method (Experiment 1; seined vs. angled fish) and within an experimental group (Experiment 2; seined fish) based on fish behaviour exhibited in the laboratory.

Second, to test for any relationship between behavioural and morphological parameters, individual traits that were strongly correlated and best represented by a single value were collapsed into a first principal component score (PC1) using Principal Components Analysis (PCA) (Table 4-1). This procedure was only performed on water column use as general activity, latency-to-exit-a-refuge, body size and body condition comprise independent, but at times correlated, behavioural variables. Across-context correlations between the PC1 score for water column use and other traits were then calculated using the nonparametric Spearman's rank correlation test. To minimize Type 1 errors in our statistical analyses, the alpha level $(\alpha=0.05)$ was adjusted to be more conservative using the sequential Bonferroni correction (Rice, 1989). 
Lastly, we used an Analysis of Covariance (ANCOVA) to compare the plasma cortisol concentrations of fish captured by seining with those captured by angling. Total body length was used as a covariate.

\section{RESULTS}

\section{Experiment 1: latency to exit a refuge and explore a novel environment}

In general, fish caught by seining were bolder, having shorter latencies to emerge from refuge $\left(Z_{1}=-4.44, P<0.0001\right)$, and had more parasites $\left(Z_{I}=-2.77, P=0.006\right)$ than fish caught via angling (Figure 4-2 a,b). However, they did not differ in general activity $\left(Z_{1}=\right.$ $0.84, P=0.401$ ), water column use (upper zone: $Z_{1}=-1.36, P=0.174$; lower zone: $Z_{1}=$ $1.32, P=0.189)$, total body length $\left(Z_{l}=-1.95, P=0.051\right)$, or body condition $\left(Z_{1}=-\right.$ $0.528, P=0.604$ ) (Figure 4-2 c,d,e,f, respectively).

Spearman rank correlation tests between latency-to-exit-a-refuge (refuge emergence) and time spent active, total body length, body condition and the PC1 score for water column use showed consistent relationships within and between experimental

groups. For all fish combined, refuge emergence was strongly negatively correlated with both time spent active $\left(r_{\mathrm{s}}=-0.31, N=134, P=0.0003\right)$ and water column use $\left(r_{\mathrm{s}}=-\right.$ $0.29, N=134, P=0.0006)$. Refuge emergence was also negatively correlated with total body length $\left(r_{\mathrm{s}}=-0.21, N=228, P=0.002\right)$, but not with body condition $\left(r_{\mathrm{s}}=0.003, N\right.$ $=228, P=0.964)$. The direction and relative strength of the above relationships were similar for both angled and seined groups, with the exception that refuge emergence and total body length were significantly negatively correlated with each other for the angled 
group $\left(r_{\mathrm{s}}=-0.30, N=109, P=0.002\right)$ but not for the seined group $\left(r_{\mathrm{s}}=-0.07, N=119\right.$, $P=0.450)$.

\section{Experiment 2: boldness and susceptibility of seined fish to angling practices}

Among fish that were seined from the lake, there was no relationship between their behaviour (e.g. refuge emergence, water column use, general activity) in Experiment 1 and whether individuals were caught when angling in the outdoor pool (range $Z_{I}=-0.91$ -0.77 , all $P>0.05$ ). However, when considering the location (open water vs. refuge) where individuals were located just prior to approaching the baited hook and being caught, fish that were in open water tended to be bolder (shorter latencies to exit the refuge, Experiment 1) than individuals that were in the refuge $\left(Z_{I}=-3.62, P=0.0003\right)$. Similarly, individuals that spent more time swimming in the bottom portion of the open arena in the laboratory (Experiment 1) were also more likely to be hiding in refuge prior to being caught in the outdoor pool (Experiment 2) $\left(Z_{I}=-2.11, P=0.035\right)$. There was no other relationship evident between morphological or behavioural variables and location prior to capture (range $Z_{l}=-1.61-1.12$, all $P>0.05$ ).

\section{Experiment 3: stress assessment of beach seining and angling protocols}

When controlling for fish body length (as a covariate), there was no significant difference in plasma cortisol levels between seined (mean \pm SD; $92 \pm 108 \mathrm{ng} / \mathrm{ml}$ ) and angled (167 \pm 133) fish (ANCOVA; $F_{1,44}=2.11, P=0.153$ ). However, larger individuals tended to 
exhibit higher cortisol levels than smaller individuals after capture $\left(\right.$ ANCOVA; $F_{1,44}=$ 4.52, $P=0.039)$, irrespective of capture method.

\section{DISCUSSION}

Our study addresses three current areas of interest in behavioural ecology, namely, animal personality, sampling biases associated with field capture techniques, and the potential impact of animal capture methods on different behavioural types (i.e., fishinginduced evolution). We sought to ascertain the nature of the relationships between these variables, if any, using the bluegill sunfish. We found that juvenile sunfish exhibited significant inter-individual differences in boldness (refuge emergence) and that these differences were strongly correlated with capture method in the wild. However, water column use and general activity did not differ between seined and angled fish. Interestingly, although the data supported our prediction that recreational angling may target individuals based on their behavioural type (i.e. bold/timid), the direction of this relationship was opposite to that expected. Sunfish that were caught using a standard angling technique were, on average, more timid and had fewer ectoparasites than individuals caught with a beach seine. However, this relationship did not carry over to our experiment in an outdoor pool, wherein bolder individuals were more likely to be angled when located in open water away from refuges than more timid individuals based on their previously assessed boldness score. Though a small number of studies have been conducted on captive populations and/or in artificial ponds/lakes (e.g. Wilson et al. 1993; Biro and Post, 2008), the current study is the first to our knowledge to have characterized 
a relationship between capture technique and individual boldness in a wild population of fish.

The current study is important for behavioural and conservation biologists as the relationship between fish personality and their susceptibility to recreational and commercial harvesting practices may result in anthropogenic selection pressures affecting the phenotypic composition of natural fish populations. At the population level, Biro and Post (2008) recently demonstrated that certain fast-growing phenotypes of rainbow trout (Oncorhynchus mykiss) that differ behaviourally from slow-growing phenotypes may be up to three times more vulnerable to commercial fishing practices and, as such, be susceptible to overexploitation and fishing-induced evolution (Uusi-Heikkila et al. 2008). Should this risk of overexploitation exist for bluegill sunfish, either through recreational or commercial fishing practices, it may have important ecological consequences for the affected populations (e.g. decreased intrinsic population growth rate) as well as the quality of the fishery (Beard and Essington, 2000). Indeed, both commercial and recreational fisheries operate for bluegill sunfish in many freshwater systems across Ontario, including Lake Opinicon, emphasizing the possibility for such exploitation to occur. In addition, should different capture techniques target individuals differentially based on behavioural type, behavioural researchers may be introducing a heretofore largely unrecognized sampling bias into their experiments (Biro and Dingemanse, 2009). Our finding that angling targets timid rather than bold sunfish is both interesting and novel, as it suggests a trend opposite in direction to our initial prediction and what little information is currently available on the relationship between personality and capture technique in fishes (Wilson et al. 1993; Biro and Post, 2008). However, in the 
current study, this relationship appears to make sense ecologically. As with normal angling practices for sport fish such as sunfish, we frequented locations in or near areas of dense cover (e.g. rocky crevices, submergent vegetation) as well as open areas when fishing in Lake Opinicon. However, whilst fish were caught via angling from all locations, more individuals did tend to be caught near refuge than in open areas, in spite of equal time spent fishing in open and refuge areas. Individuals caught in or near refuges may be more timid than individuals caught in open water, as juvenile fish commonly use such cover for predator avoidance (Godin, 1997). Juvenile sunfish in particular are known to exhibit high site fidelity for these types of locations prior to reaching larger body sizes (McCairns and Fox, 2004; Wilson and Godin, 2009). In contrast, a large beach seine captures individuals from open areas as well as those in refuge, which is why it is a recommended technique for avoiding sampling biases associated with personality traits (Wilson et al. 1993; Biro and Dingemanse, 2009). This assertion is also supported by our outdoor pool experiment (Experiment 2), which revealed that bolder fish (in terms of refuge emergence) tended to be located in the open water, whereas more timid fish tended to be located near the refuges prior to capture. Since our measure of boldness had to do with individual tendency to exit a refuge, the finding that timid individuals were more likely to be caught when angling near refuge may not be so counterintuitive. As such, this finding has important implications for the manner by which species such as sunfish are caught for behavioural research, as well as for understanding the potential impact of intensive angling on the behavioural and phenotypic composition of fish populations. 
Although we cannot eliminate the possibility that environmental or experimental factors may have influenced our findings with respect to the relationship between capture technique and boldness, the results do not appear to be related to different stress levels associated with angling or seining. We found no significant difference in plasma cortisol level between the two capture-method groups when controlling for body size (Experiment 3). Indeed, when exposed to even severe stress associated with fisheries capture, physiological recovery in fish is generally quite rapid, with most physiological parameters returning to baseline values within 4 to $6 \mathrm{~h}$ of capture (Barton, 2002; Cooke and Suski, 2005).

The relationships between boldness (latency-to-exit-a-refuge), water column use and general activity were consistent and did not differ between the two capture groups, though the magnitude of these relationships did vary between seined and angled fish. Bolder individuals tended to spend more time near the water surface and were more active in general than more timid individuals. These relationships are consistent with other recent studies of boldness in juvenile bluegill sunfish in another similar, but unconnected, southern Ontario lake (Wilson and Godin, 2009, 2010; Chapters 2 and 3). While it has been suggested that correlations between particular behavioural traits should, and likely do, vary between locales (Dingemanse et al. 2007), evidence also suggests that certain selective agents (e.g. predation) can shape behavioural correlations in a predictable manner (Bell, 2005). The consistency observed between the results of the current study and others (Wilson and Godin, 2009, 2010; Chapters 2 and 3) on juvenile bluegill sunfish may be a result of similar predation pressures within each study locale, similar environmental factors due to their close geographical proximity, or some 
combination of these and other factors. This finding may provide important insight into gaining an understanding of the nature and geographic distribution of sunfish boldness traits and how they have evolved in Ontario lakes.

The current study describes, for the first time, a relationship between boldness and recreational angling practices in fishes. Since juvenile bluegill sunfish are primarily prey fish for larger piscivorous fishes (e.g. largemouth bass, Micropterus salmoides; northern pike, Esox lucius; Scott and Crossman, 1998), the implications of our findings for predatory species as well as for individuals of different developmental stages (i.e. adult vs. juvenile) remain subjects in need of further investigation. That said, our findings do represent important evidence for a potential sampling bias associated with capture technique, and perhaps location of capture, and thus should be of particular interest to behavioural biologists. 


\section{ACKNOWLEDGEMENTS}

We thank C. O' Conner for assistance in processing plasma cortisol samples, and $\mathrm{K}$. Gilmour for the use of her equipment and materials in the analysis of these cortisol samples. We also thank the staff at the Queen's University Biological Station for technical support and the Ministry of Natural Resources of Ontario for the necessary fish collection permit. This study was approved by the institutional Animal Care Committee at Carleton University (protocols B06-7; B09-10) and thus adheres to the guidelines of the Canadian Council on Animal Care and the laws of Canada. This research was supported by the Natural Sciences and Engineering Research Council of Canada (Discovery research grants to J.-G.J.G. and S.J.C and a post-graduate scholarship to A.D.M.W.). S.J.C. is also supported by the Canada Research Chairs program. 
Table 4-1: PCA loadings of behavioural variables used to generate a principal component score (PC1) for water column use and other behavioural and morphological traits in juvenile bluegill sunfish.

\begin{tabular}{llrc}
\hline Trait & Behavioural variables & $\begin{array}{c}\text { Loadings for } \\
\text { PC1 }\end{array}$ & $\begin{array}{c}\% \\
\text { variation } \\
\text { explained }\end{array}$ \\
\hline \multirow{2}{*}{ Water column use } & Time spent in upper zone & & \\
& Time spent in lower zone & 0.7071 & 71.9 \\
\hline
\end{tabular}




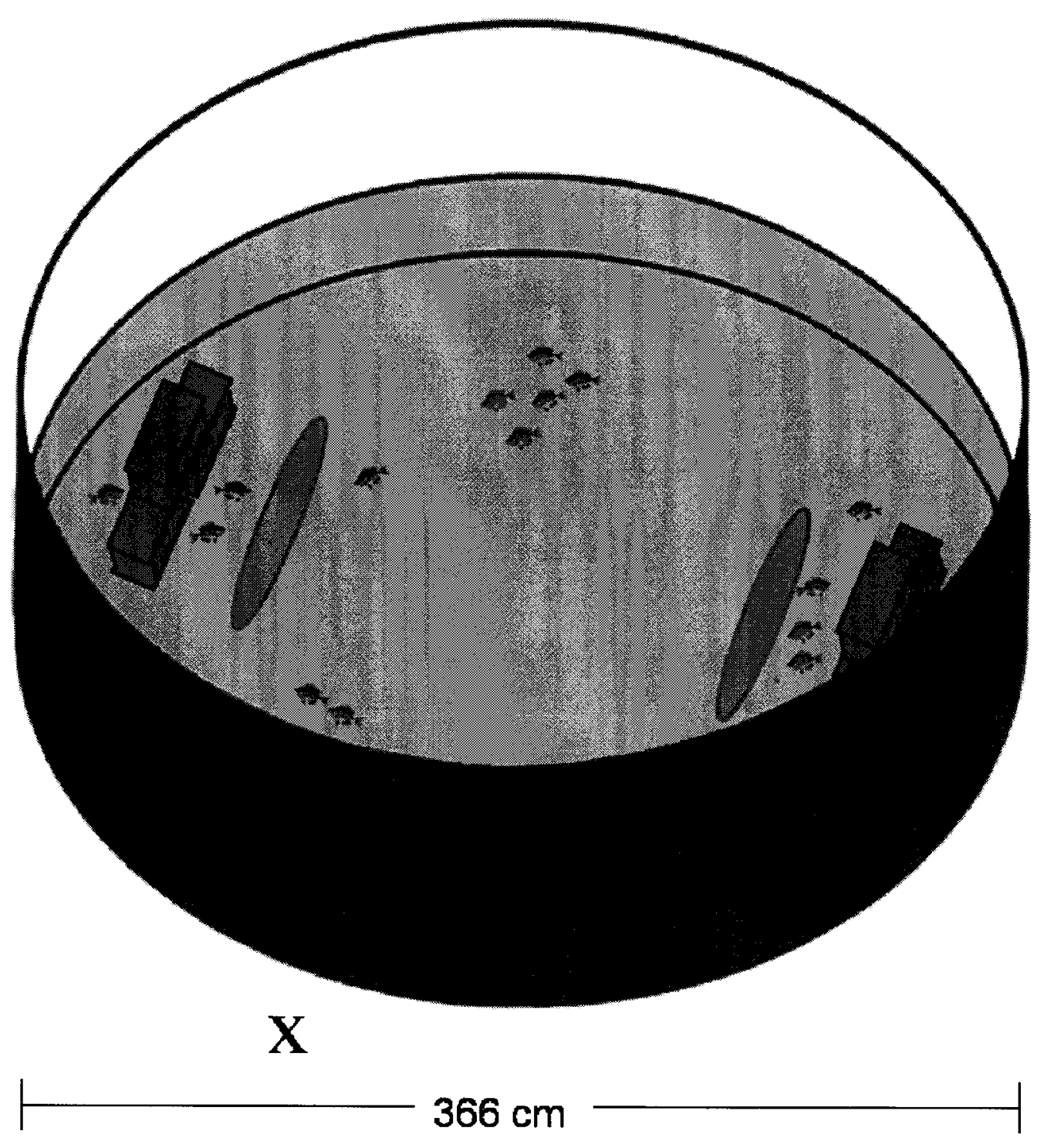

Figure 4-1: Schematic representation of the experimental pool tank used in Experiment 2 to quantify the susceptibility of seined fish to angling practices based on their individual boldness score obtained from Experiment 1. The red ovals represent the areas wherein the baited hook was alternately positioned on each side of the tank during experimental trials. The stacked cinderblocks provided refuge for the fish. The $\mathbf{X}$ denotes the position of the angler-observer. 

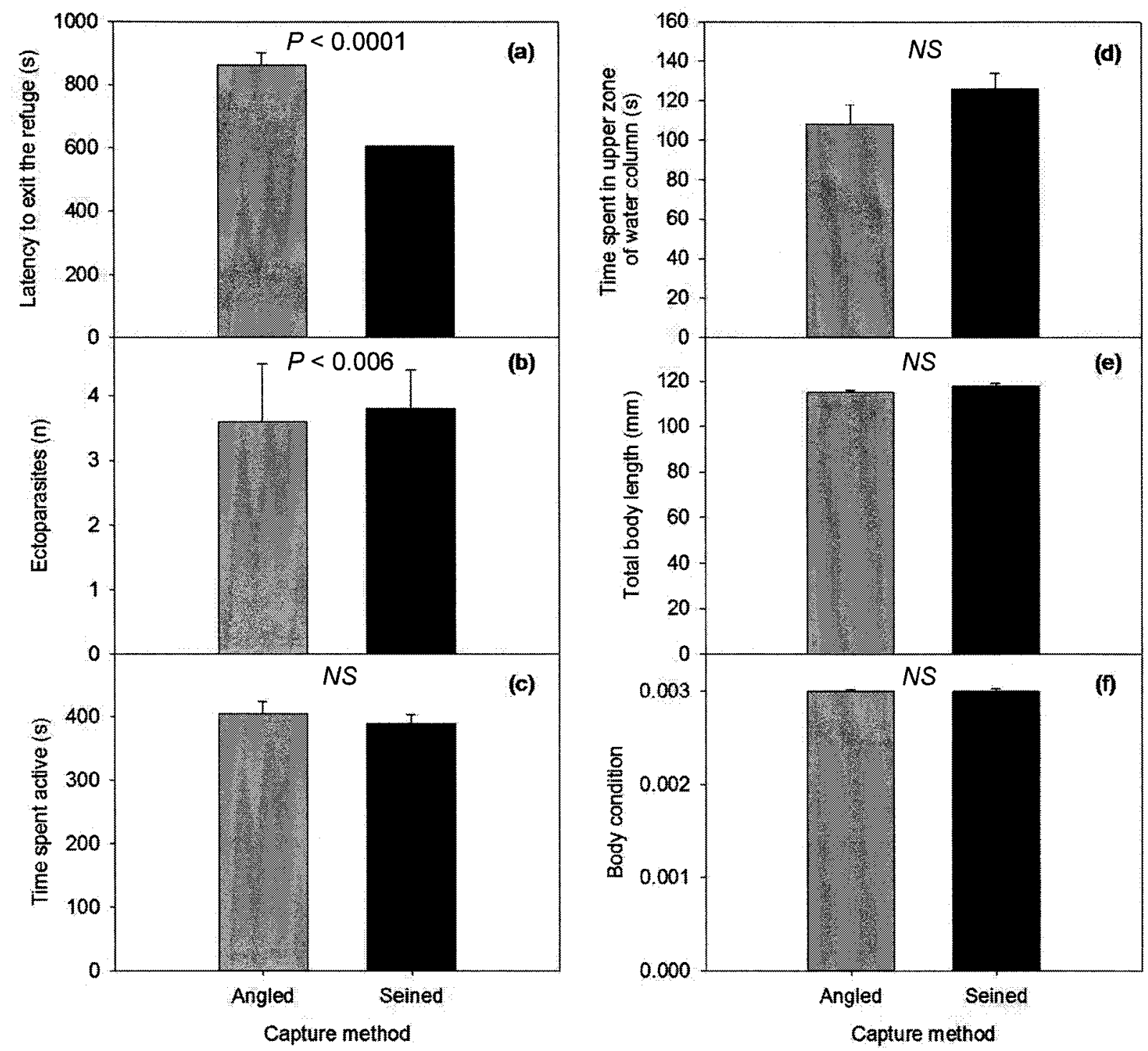

Figure 4-2: Mean ( $\pm \mathrm{SE}$ ) refuge emergence (a), ectoparasites (b), general activity (c), time spent in upper zone of the water column (d), total body length (e), and body condition (f) in juvenile bluegill sunfish caught by either angling or beach seining capture techniques. Gray bars represent individuals caught via angling; black bars represent individuals caught via seining. $P$-values shown were obtained using the Kruskal-Wallis ANOVA. NS denotes a non-significant difference between the means being compared. 


\title{
CHAPTER 5
}

\section{BOLDNESS AND REPRODUCTIVE FITNESS CORRELATES IN}

\author{
THE EASTERN MOSQUITOFISH, GAMBUSIA HOLBROOKI *
}

* Published in Ethology

Wilson ADM, Godin J-GJ, Ward AJW, 2010. Boldness and reproductive fitness correlates in the Eastern mosquitofish, Gambusia holbrooki. Ethology 116: 96104. 


\begin{abstract}
Animals are known to exhibit 'personality'; that is, individual differences in behaviour that are consistent across time and/or situations. One axis of personality of particular importance for behavioural ecology is boldness, which can be defined as the tendency of an individual to take risks. The relationship between individual personality and fitness correlates, particularly those involved in reproduction, remains largely unexplored. In the current study, we used female Eastern mosquitofish (Gambusia holbrooki) to ascertain whether certain reproductive traits (e.g. stage of pregnancy, fecundity) are correlated with individual personality in two wild populations in New South Wales, Australia. To quantitatively assess this relationship, we tested individual fish for their level of boldness, as measured by their latency to exit a refuge and tendency to shoal, general activity, and tendency to swim near the water surface and substratum in a novel laboratory environment. For both populations taken together, bolder individuals tended to be smaller, relatively less fecund (when taking body size into account), and spent more time near the water surface than near the substratum compared with timid individuals.

Individual boldness was not correlated with either general activity or stage of pregnancy. To our knowledge, our study characterizes for the first time a relationship between an individual personality trait (boldness) and a reproductive fitness correlate (fecundity) in fishes.
\end{abstract}




\section{INTRODUCTION}

The concept of animal 'personality', or individual level differences in behaviour that are consistent across time and/or situations, has become a subject of much research in recent years (Réale et al. 2007). This is particularly true in terms of understanding the mechanisms underlying the origin, evolution and fitness consequences of animal personality (Gosling, 2001; Sih et al. 2004; Dingemanse and Réale, 2005; Wolf et al. 2007; Smith and Blumstein, 2008). However, many questions remain unanswered, including whether individual differences in morphological, physiological, or life-history traits can be predicted by individual differences in personality or vice versa. Further, individual differences in personality may have profound implications for behavioural ecologists as they have been documented to be associated with a broad range of ecologically relevant behaviours (e.g. mating, foraging, habitat use, exploration, antipredator behaviour) (Dingemanse and Réale, 2005; Réale et al. 2007) and because their importance is often ignored when conducting field research (Biro and Dingemanse, 2009).

One axis of personality of particular relevance to behavioural ecology is that of "shyness" and "boldness". Boldness can be defined as the tendency of an individual to take risks (Wilson et al. 1994). Along the shy-bold axis (Wilson et al. 1994), individuals vary from being extremely bold (reacting to novel stimuli by becoming actively exploratory) to extremely shy or timid (reacting to novel stimuli by retreating or becoming vigilant). The ecological importance of boldness is apparent in its broad taxonomic expression and the range of other behaviours it is associated with. Boldness has been widely documented in fishes (Ward et al. 2004; Webster et al. 2007; Wilson and 
Godin, 2009; Chapters 2-4 above), birds (Dingemanse et al. 2003; Carere and van Oers, 2004; Both et al. 2005; ), mammals (Réale and Festa-Bianchet, 2003; Martin and Réale, 2008;), reptiles (Lopez et al. 2005), insects (Kortet et al. 2007, Wilson et al. 2009), and cephalopods (Sinn et al. 2006; 2008). Further, boldness has also been shown to be associated with aggression (Johnson and Sih, 2005), mate selection (Godin and Dugatkin, 1996), dispersal (Fraser et al. 2001), invasiveness (Rehage and Sih, 2004), general activity, exploration and space use (Wilson and Godin, 2009), as well as foraging and antipredator behaviour (Wilson and Stevens, 2005; Jones and Godin 2010). Boldness has also been posited to be associated with traits that are fitness-related (Dingemanse et al. 2004; Sinn et al. 2006), is heritable (Dingemanse et al. 2002; Brown et al. 2007a), and subject to natural selection (Réale and Festa-Bianchet, 2003). Thus, investigating the ecological and evolutionary implications of shy-bold behaviour, as well as its mechanistic bases, may provide important information on how these individual differences arise and are maintained in natural populations.

Here, we used female Eastern mosquitofish (Gambusia holbrooki) to ascertain whether certain reproductive traits (e.g. reproductive stage, fecundity) are correlated with boldness and other behavioural (general activity, water column use) and morphological (e.g. body size) traits in two wild populations in New South Wales, Australia.

To quantitatively assess the relationship between reproductive traits and personality (i.e. boldness) in individuals, we captured adult female mosquitofish from two populations and tested them for individual differences in risk-taking behaviour and general exploratory activity under standardized laboratory conditions. Following behavioural trials, we measured morphological traits (e.g. total length, wet weight) and 
dissected each fish to ascertain the stage of development and number of embryos each individual was carrying. Should fecundity/pregnancy be correlated with boldness, we predicted that females carrying more embryos and/or embryos closer to parturition (later stage of development) would be bolder than females with fewer embryos or embryos earlier in development due to greater associated metabolic requirements and/or greater embryonic investment (Koch and Wieser, 1983; Marsh-Matthews et al. 2001; Lindström et al. 2003). Characterizing the relationship between reproductive status/traits and boldness should contribute to furthering our understanding of the evolution of personality in animals.

\section{METHODS}

\section{Subjects}

On April 7-8 and 17-18, 2008, we used two large (1-m diam.) dip nets to capture adult female (total length: $2.8-4.4 \mathrm{~cm}$; wet weight $0.14-1.02 \mathrm{~g}$ ) mosquitofish from the littoral zone of two temperate lakes in New South Wales, Australia. Our first capture site, Lake Northam, is a small $\left(\approx 550 \mathrm{~m}^{2}\right.$ surface area) body of water in Victoria Park, Sydney (33 53 '6"S; $\left.151^{\circ} 11^{\prime} 35^{\prime \prime} \mathrm{E}\right)$. Our second capture site was a small bay in the comparatively larger $\left(\approx 260000 \mathrm{~m}^{2}\right.$ surface area) Manly Dam Reservoir (3346’38”S; $151^{\circ} 15^{\prime} 3$ 'E). At the time of capture, both field sites possessed a variety of submergent and emergent vegetation and high densities of mosquitofish were observed.

All captured females were placed in buckets containing lake water and transported to our laboratory at the University of Sydney (maximum transit time $<30$ min). On each of the two-day periods above, one day was spent collecting at Lake 
Northam and the second day was spent collecting at the Manly Dam Reservoir (hereafter referred to as Manly Dam).

\section{Experimental holding conditions}

On arrival at the laboratory, all fish caught from a given location and on a given day were housed communally, but separate from those captured on different days, in one of two large glass aquaria ( $95 \mathrm{~L} ; 60 \mathrm{~cm} \times 45 \mathrm{~cm} \times 35 \mathrm{~cm}$ ). Each aquarium contained aerated and filtered dechlorinated tap water maintained at $25 \pm 1^{\circ} \mathrm{C}$, and illuminated by overhead

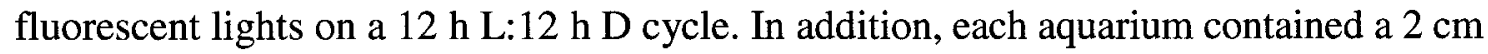
layer of natural coloured aquarium gravel as a substratum. During the holding period, fish were fed commercial tropical fish flakes ad libitum twice daily.

All fish were held for 2-3 weeks prior to the onset of behavioural testing to allow for a broad range of pregnancy stages to arise. During testing, individuals were selected from the one of the two holding tanks under a standardized protocol, such that the same number of females from each population was tested alternately every other day (e.g. day $1=10$ individuals from Lake Northam; day $2=10$ individuals from Manly Dam; etc.), beginning 2 wks after capture. In total, 100 fish (50 from each population) were tested individually and sequentially in both Experiments 1 and 2.

\section{Quantifying boldness behaviour and exploratory activity}

We characterized boldness in individual fish using two behavioural measures (latency to exit a refuge, and tendency to shoal with conspecifics) in two separate corollary 
experiments, as described below. In addition, we concurrently quantified the general exploratory activity of focal fish in a novel environment (as time spent near the water surface or near the substratum, and time spent active).

\section{Experiment 1: latency to exit a refuge and explore a novel environment}

The experimental arena used for this experiment consisted of a rectangular aquarium (30 $\mathrm{L}, 40 \times 25 \times 30 \mathrm{~cm}$ ) that had been 'divided' horizontally into four equal zones (two vertically and two horizontally) using lines drawn on the front and back walls to facilitate the observation of fish water column use. The tank was covered with opaque black plastic on the back and sides to prevent any outside stimulus from disturbing the fish. The aquarium was placed behind an opaque plastic blind equipped with a small window (20 $\mathrm{cm} \times 20 \mathrm{~cm}$ ) for observing fish behaviour remotely. The experimental tank contained a mix of new aged water and water from the appropriate holding tank, which was replaced between trials. Located at one end of the experimental arena was a 'refuge' that consisted of an upright PVC cylindrical tube (inside diameter: $7.5 \mathrm{~cm}, 0.25 \mathrm{~cm}$ thick) with a rectangular door $(3.5 \times 4.5 \mathrm{~cm})$ cut into the bottom portion. This tube was fixed to the bottom substratum, which consisted of $2 \mathrm{~cm}$ of natural coloured aquarium gravel. This first tube was a placed inside a second larger PVC tube (inside diameter: $8.5 \mathrm{~cm} ; 0.25 \mathrm{~cm}$ thick) that also had a rectangular door $(4 \times 5 \mathrm{~cm})$ cut into its bottom portion. Prior to the onset of testing, the doors of each tube were kept offset to each other, such that the inner opening was held closed and the focal fish could not exit. At the onset of a trial, the outer tube was turned clockwise such that the two doors lined up and the fish could exit the refuge area. Just outside the tube, and visible from the refuge, was a plastic aquarium 
plant $(20 \mathrm{~cm}$ tall) anchored into the substratum. The planted area comprised approximately $10 \%$ of the open arena.

Just prior to the beginning of a behavioural trial, a focal fish was haphazardly dipnetted from the appropriate holding aquarium, placed in a small container $(1 \mathrm{~L})$ containing water from its holding tank, and promptly transported to the novel experimental aquarium. The focal fish was slowly poured into the refuge within the aquarium. The fish was then given $10 \mathrm{~min}$ to acclimatize to its surroundings, prior to the onset of testing for its willingness to explore the novel environment. After this period, the refuge door was opened and the focal fish was given a further $10 \mathrm{~min}$ to exit the refuge and explore the open arena. Where the fish exited completely within $10 \mathrm{~min}$, we recorded the latency time to exit and subsequently quantified several behavioural measures for a further $10 \mathrm{~min}$. These variables were: (i) total time spent in the upper $(15 \mathrm{~cm}$ nearest surface) and lower (15 cm near substratum) zones of the water column, and (ii) total time spent being active in the open arena. Fish that did not exit the refuge were assigned a maximal latency-to-exit score of $10 \mathrm{~min}$ and no further behavioural measures were recorded for those individuals. Activity was defined as the total time spent swimming (vs. resting on the bottom or holding position in the water column) in the open arena during the $10-$ min observation period. Because we were interested primarily in exploration and risk-taking tendencies, only behaviour exhibited in the open arena, and not inside the refuge (prior to, or upon volitional re-entry during the course of a trial), was quantified. All behavioural variables were quantified by a single observer (ADMW) using the behavioural analysis software JWatcher (v1.0) (Blumstein and Daniel 2007). 
Following the 10-min behavioural observation period, the focal fish was removed (by dipnetting) from the experimental aquarium and immediately carried over to the second phase of experimentation (Experiment 2). Approximately 10 fish were similarly tested each day of experimentation (for a total of 100 fish).

\section{Experiment 2: shoaling tendency}

Immediately following the conclusion of Experiment 1, each focal female $(n=100$; same individuals as in Experiment 1) was placed separately and singly into a second novel experimental arena, given 5 min to acclimatize, and then tested individually for their shoaling tendency. As for Experiment 1, this experimental aquarium ( $15 \mathrm{~L}, 36 \times 20 \times 20$ $\mathrm{cm}$ ) was covered on the sides and back with opaque black plastic and was located behind an observation blind. The aquarium was divided into two adjacent compartments by a transparent, perforated partition, which allowed both visual and olfactory cues to be transmitted between compartments. The smaller of the two compartments $(8.5 \times 20 \times 20$ $\mathrm{cm}$ ) contained a stimulus shoal consisting of four females (two from Lake Northam, two from Manly Dam) matching the size range of focal fish being tested. Throughout all behavioural trials, the same four fish were used as a stimulus shoal and were held separately from the main holding tanks, though under similar holding conditions. The second, larger compartment $(27.5 \times 20 \times 20 \mathrm{~cm})$ was 'divided' lengthwise into nine distinct zones by vertical lines drawn every $3 \mathrm{~cm}$ (approx. equivalent to 1 body length) on the front wall. This compartment contained the focal test fish. The bottom of the experimental arena was uniformly covered with $2 \mathrm{~cm}$ of natural coloured aquarium gravel. 
Shoaling tendency was quantified by recording the amount of time the focal fish spent in each of the nine zones of the larger compartment over a period of $3 \mathrm{~min}$.

Individuals that spent more time swimming at least three body lengths ( $>3$ zones) away from the stimulus shoal were considered to be bolder (i.e. taking greater risks) than those individuals that spent more time closer to the partition/stimulus shoal ( $\leq 3$ zones). In nature, members of fish shoals are typically within three body lengths of their nearest neighbour (Pitcher and Parrish 1993). Shoals offer safety from predation, and an individual that moves away from a shoal exposes itself to a greater risk of predation than otherwise (Krause and Ruxton, 2002). All behavioural variables were quantified by a single observer (ADMW) using JWatcher (v1.0).

Immediately following the 3-min trial period, the focal fish was captured, euthanized using clove oil, and stored in $95 \%$ ethanol for later morphometric measurements and dissection.

\section{Quantifying morphological and reproductive traits}

Following the completion of the behavioural trials, all fish were individually weighed and standard morphological measurements (e.g. total length) were taken. Each female was then dissected and the number and developmental stage of her embryos were quantified. The different stages of embryonic development were categorized based on a classification scheme (Table 5-1) modified from the one described by Reznick (1981). 


\section{Data analyses}

In the current study, both boldness and water column use each comprised two independent but correlated behavioural variables. Consequently, latency to exit the refuge and time spent away from a shoal were entered into a Principal Components Analysis (PCA) and the first principal component (PC1) was used as a single composite score to characterize the boldness level of individual female mosquitofish, for both populations collectively. In addition, time spent near the water surface (upper water column zone) and time spent near the substratum (lower zone) were collapsed into a separate single composite score (PC1) to characterize water-column-use behaviour of individual females (see Table 5-2). Time spent in both the upper and lower zones were used in the principal component (PC1) score for water-column-use as individuals could re-enter the refuge on their own volition during trials, and this refuging behaviour was not quantified. Therefore, time spent in each zone may not be the exact inverse of each other for all individuals.

These two principal component scores and a general activity score (time spent active in the open arena) for each fish were then separately compared with their morphological and reproductive traits using nonparametric Spearman's rank correlation tests (df = 97 for each test, sequential Bonferroni correction applied (Rice 1989). Because the relationship between absolute fecundity and female body length overall was positive and linear $\left(r^{2}=0.63, n=100, P<0.0001\right)$ in the current study (more specifically, Lake Northam population, $\mathrm{r}_{\mathrm{s}}=0.72, \mathrm{df}=48, \mathrm{p}<0.0001$; Manly Dam population, $\mathrm{r}_{\mathrm{s}}=$ $0.90, \mathrm{df}=48, \mathrm{p}<0.0001$ ), we calculated 'relative fecundity' for each female as the ratio 
of the number of eggs/embryos she carried to her total body length to control for differences in body size between individuals and populations.

Two fish (out of 100 tested) were identified as statistical outliers using the extreme studentized deviate (ESD) method and were thus removed from our statistical analyses. All statistical tests were carried out using JMP (v. 4.0.4).

\section{RESULTS}

Overall, the boldness (PC1) score of individual females was negatively correlated with both their body length $\left(r_{s}=-0.37, p<0.001\right.$; Fig. 5-1a) and relative fecundity $\left(r_{s}=-0.31\right.$, $p=0.003$; Fig. 5-1b), and strongly positively correlated with their water-column-use behaviour $\left(r_{s}=0.43, p=0.0003\right.$; Fig. 5-1c). Boldness was not, however, correlated with either activity $\left(r_{s}=-0.04, p=0.76\right.$; Fig. $\left.5-1 d\right)$ or stage of pregnancy $\left(r_{s}=0.05, p=0.68\right)$. These trends were consistent across the two study populations (Fig. 5-1). So, for both populations taken together, bolder individuals tended to be smaller, relatively less fecund (when taking body size into account), and spent more time near the water surface than near the substratum compared with timid individuals.

\section{DISCUSSION}

Our study addresses two current areas of interest in behavioural ecology, namely, animal personality and reproductive/fitness correlates in wild animal populations. Here, we sought to ascertain the nature of the relationship between these two types of trait in mosquitofish originating from two populations. Most importantly, we found that smaller 
female mosquitofish tended to be bolder than larger conspecifics under standardized experimental laboratory conditions. When controlling for inter-individual variation in body length, the fecundity (expressed as relative fecundity) of individual females was negatively correlated with their boldness score. Contrary to our expectations, boldness was not correlated with stage of pregnancy nor general activity. That said, the observed relationship between boldness and relative fecundity suggests that reproductive investment influences boldness behaviour, independent of individual body size. However, the direction of this relationship between boldness and fecundity was opposite to the one which we predicted a priori. To our knowledge, the current study is the first to have characterized the relationship between a reproductive fitness correlate (fecundity) and boldness in fishes, and among only a few to date to have attempted to describe similar relationships between personality and reproductive fitness in other taxa (e.g. Réale et al. 2000; Stapley and Keogh 2005; Boon et al. 2007).

We also observed that the water-column-use behaviour of female mosquitofish was positively correlated with their boldness score; that is, bolder individuals tended to spend more time near the water surface (than near the substratum) than more timid individuals. Water column use and general activity have been previously recognized as meaningful measures of risk-taking behaviour in fishes (e.g. Wilson and McLaughlin, 2007; Wilson and Godin, 2009, Chapter 2), as presence near the water surface and increased activity can elevate the risk of predation from both aerial and aquatic predators (Lima and Dill, 1990; Godin, 1997; Sih et al. 2003). However, in mosquitofish, time spent near the water surface may not reflect a greater willingness to take risks, as this species is generally a surface-dwelling taxon (Pyke 2005). Water column use by 
mosquitofish may be more indicative of foraging or general exploratory tendencies. Both foraging and exploration have been shown to be strongly associated with risk-taking behaviour (see Lima and Dill 1990; Sih et al. 2004; Réale et al. 2007), and our finding of a strong positive correlation between boldness and water column use further supports this association. However, contrary to our initial expectation, individual boldness behaviour and general activity were not significantly correlated in the current study. Based on studies of other species (e.g. Sneddon 2003; Reaney and Backwell 2007; Wilson and Godin 2009, Chapter 2), we had expected that bolder individuals would be more active than more timid ones. It is possible that the relationship between boldness and activity may differ between pregnant/reproductive and non-pregnant/non-reproductive individuals, and that this may explain the difference between our finding and those of other studies on the nature of this particular relationship.

The level of parental investment of resources, both temporal and physiological, in bringing embryos to parturition (Marsh-Matthews et al. 2001; Lindström et al. 2003;) may play an important role in determining the willingness of a female to take risks in novel situations. High fecundity and embryos close to parturition both represent a significant energetic investment by females and may be associated with increased metabolic demand in pregnant individuals, making them more willing to take risks to forage so as to meet those demands. Conversely, high fecundity and later-stage embryos could render pregnant females less willing to take risks (i.e. more cautious) and, in doing so, minimizing their risk of mortality to predation, thereby safeguarding their current and future reproductive investment in offspring. Indeed, our results support this latter proposition as more timid female mosquitofish typically had greater relative fecundity 
than bolder ones. Thus, individual differences in boldness may be affected differentially depending on proximity to parturition and overall current embryonic investment. More importantly, the association between individual boldness and relative fecundity exists irrespective of body size in mosquitofish. This novel finding further suggests that pregnancy influences an individual female's boldness, which hitherto has been a largely ignored avenue of animal personality research (e.g. Réale et al. 2000; Stapley and Keogh, 2005).

Refuge emergence is also a common measure of boldness in many species, including fishes (e.g. Brown and Braithwaite, 2004; Wilson and Godin, 2009; Chapter 2). In the current study, it constituted one component measure of the composite boldness score for individual fish. Poor or uncertain information about the environment outside of a refuge can pose a significant risk with regard to predation and also poses a constraint on 'prey' to respond adaptively to ambient conditions (Sih, 1992, 1997). As such, the decision of an individual to emerge from a refuge involves the assessment of a number of habitat variables (e.g. food availability, predation threat) and should be fairly robust across populations (Brown and Braithwaite, 2004). In our study, female refugeemergence behaviour was strongly correlated with both relative fecundity and body size. Larger female mosquitofish took longer to emerge from a refuge than smaller individuals, and more timid individuals tended to have higher fecundity independent of overall body size than larger conspecifics. These findings corroborate those of recent studies on refuge-emergence behaviour in another poeciliid fish (Brown and Braithwaite, 2004; Brown et al. 2005, 2007b), wherein smaller individuals tended to be bolder. Brown and Braithwaite (2004) hypothesized that this trend towards smaller fish leaving refuge 
sooner may be a result of energetic factors associated with metabolic demands and life history stage. Alternatively, individual differences in female boldness may be related to pregnancy, with more timid fish behaving as such due to greater embryonic investment, as suggested by our current results.

Although we found that the nature (direction) of the relationships between individual boldness and certain behavioural and reproductive traits were consistent across our two study populations (Fig. 5-1), the strength of some of these relationships varied between them. The factors potentially generating such variation between our two study populations are unknown. Dingemanse et al. (2007) postulated that correlations between particular behavioural traits may vary with ambient conditions at particular locales, such that few overarching trends may be revealed across populations and taxa. That said, certain selective agents (e.g. predation) do appear to shape behavioural correlations in a predictable manner (Bell, 2005). Indeed, the strong relationships between boldness, water column use, and fecundity observed across and within populations in the current study are consistent with other within-population studies on behavioural correlations (Ward et al. 2004; Wilson and McLaughlin, 2007; Wilson and Godin, 2009; Chapter 2) and suggest that such relationships between behavioural and morphological/reproductive traits may be subject to selection in nature.

In conclusion, our study characterizes for the first time a relationship between an individual personality trait (boldness) and a reproductive fitness correlate (fecundity) in fishes. Given such a relationship, further examination of the potential influence of pregnancy and other reproductive traits on boldness may offer important insights into the underlying mechanistic bases and ecological relevance of this and other personality traits. 
However, the exact nature of this relationship and its utility as a predictor of boldness in other populations remain a subject for further investigation.

\section{ACKNOWLEDGEMENTS}

Our study was approved by the institutional Animal Care Committee at the University of Sydney (protocol \#L04/3-2008/1/4768) and thus adheres to the laws of Australia. This research was funded by an NSERC of Canada discovery research grant to J.-G.J.G. and a University of Sydney grant to A.J.W.W. A.D.M.W. was funded by an NSERC postgraduate scholarship. 
Table 5-1: A comparison of the stages of classification for Eastern mosquitofish embryonic development, as described by Reznick (1981) and used in the current study.

\begin{tabular}{ccl}
\hline $\begin{array}{c}\text { Current study } \\
\text { (stage \#) }\end{array}$ & Reznick (1981) & \multicolumn{1}{c}{ Description } \\
\hline 1 & No development & $\begin{array}{l}\text { No development readily discernible under a } \\
\text { dissecting microscope. }\end{array}$ \\
\hline 2 & Uneyed & $\begin{array}{l}\text { Embryo is seen as a "pale ribbon" on the } \\
\text { surface of the ovum. }\end{array}$ \\
\hline 3 & Early-eyed & $\begin{array}{l}\text { Some retinal pigment, little or no body } \\
\text { pigment. }\end{array}$ \\
\hline 4 & Mid-eyed & $\begin{array}{l}\text { Melanophores appear on the dorsum, fin } \\
\text { rudiments present, and the yolk is reduced in } \\
\text { size. }\end{array}$ \\
\hline 6 & Late-eyed & $\begin{array}{l}\text { Melanophores present over the surface of the } \\
\text { body, fin rays present, yolk size substantially } \\
\text { reduced. }\end{array}$ \\
\hline 6 & Very late-eyed & $\begin{array}{l}\text { Fins are fully formed and yolk is virtually } \\
\text { absent. }\end{array}$ \\
\hline
\end{tabular}


Table 5-2: PCA loadings of behavioural variables used to generate a principal component score (PC1) to assess correlations between boldness, water column use, and other behavioural, morphological, and reproductive traits in female mosquitofish.

\begin{tabular}{llrc}
\hline Trait & Behavioural variables & $\begin{array}{c}\text { Loadings for } \\
\text { PC1 }\end{array}$ & $\begin{array}{c}\% \\
\text { variation } \\
\text { explained }\end{array}$ \\
\hline \multirow{2}{*}{ Boldness } & Latency to emerge from refuge & -0.7071 & 50.6 \\
& Time spent away from shoal ( $>3$ zones) & 0.7071 & \\
& & & \\
Water column use & Time spent in upper zone & -0.7071 & 99.9 \\
& Time spent in lower zone & 0.7071 & \\
\hline
\end{tabular}



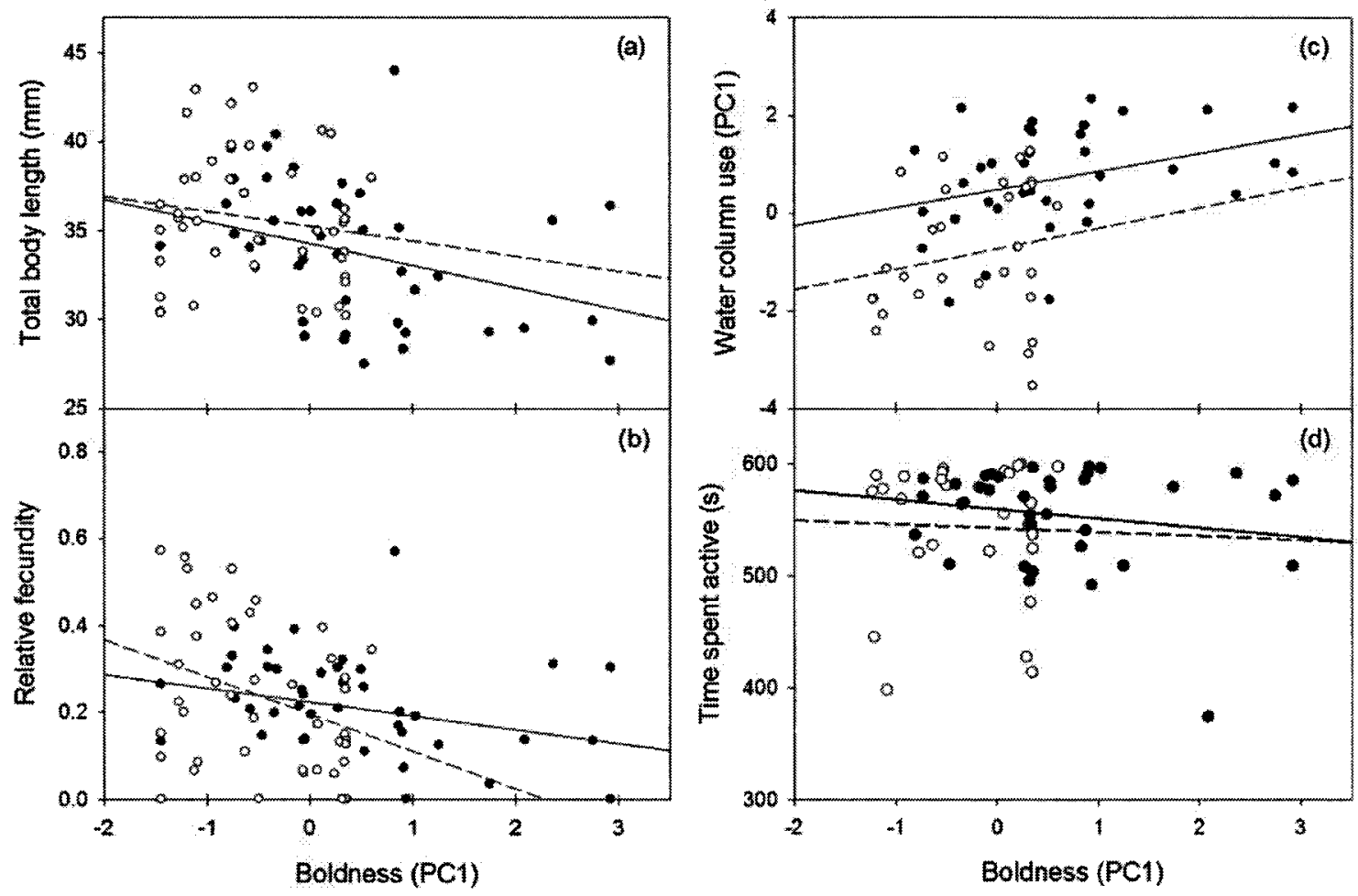

Figure 5-1: Observed individual differences in (a) total body length, (b) relative fecundity (= number of eggs or embryos / total body length), (c) water column use, and (d) time spent active in relation to boldness in female mosquitofish. Each data point represents an individual fish $(\mathrm{n}=98)$. Open circles $(0)$ and dashed lines represent values for individuals collected from Lake Northam. Closed circles $(\bullet)$ and solid lines represent values for individuals collected from Manly Dam. Each line denotes a line-of-best-fit through the relevant data obtained from a least-square regression to illustrate the trend in the data. 


\section{CHAPTER 6}

\section{GENERAL CONCLUSIONS}

\section{Synthesis}

Individual-level differences in personality traits (e.g. boldness, aggression, sociability) are increasingly being recognized as important areas of behavioural research, as they incorporate many aspects of an animal's behavioural repertoire (Réale et al. 2007; Wolf et al. 2008). Further, these traits are known to be associated with many ecologicallyrelevant behaviours (e.g. dispersal, foraging, mating) and to be present in a wide range of taxa (Réale et al. 2007). Thus, investigating the nature and mechanistic bases by which individual differences in personality arise and are maintained in wild populations represents an opportunity to understand the ecological and evolutionary implications of behaviour across taxa. The aim of my thesis research was to investigate some aspects of the behavioural ecology of boldness in fishes. To accomplish this goal, I conducted experiments that incorporated multiple avenues of important, and heretofore largely underappreciated, areas of boldness research. These avenues are (a) behavioural syndromes, (b) animal locomotion, (c) anthropogenic influence and (d) reproductive fitness correlates.

In Chapter 2, I investigated whether juvenile bluegill sunfish exhibit interindividual differences in boldness using the conceptual framework of behavioural syndromes. Wild-caught sunfish were tested in standardized boldness experiments over several days in several different behavioural contexts. I also tested whether boldness was repeatable over a two-month mark-recapture period in the wild. I predicted that boldness 
would be both consistent and repeatable across contexts. Bluegill sunfish were observed to exhibit consistent and repeatable differences in behaviour, which likely represent a boldness syndrome that incorporates aspects of risk-taking, activity and exploration. Refuge emergence, a behavioural trait representing exploration tendency and willingness to enter a potentially risky environment, played a prominent role in this syndrome. The usefulness of this behavioural trait for future behavioural studies was apparent from both the highly consistent nature of its expression across behavioural tests and its repeatability in individuals following the two-month recapture period. It was because of its consistency and repeatability, as well as its strong correlations with other behavioural traits (activity, antipredator behaviour) that I chose to use refuge emergence as a standardized measure of willingness to take risks and explore in subsequent experiments (Chapters 3-5). This chapter illuminated for the first time the nature of a boldness syndrome and its temporal repeatability and consistency in a wild fish, the bluegill sunfish.

In Chapter 3, I characterized the relationship between intermittent locomotion and boldness in juvenile bluegill sunfish. Intermittent locomotion in an animal consists of bursts of movement punctuated by stationary pauses of variable duration (Kramer and McLaughlin 2001). I used frame-by-frame video analysis to quantify individual differences in distinct undulatory (fast) and labriform (slow) swimming modes whilst exploring a novel environment. Individual differences in intermittent locomotion in sunfish covaried with individual differences in boldness (refuge emergence, water column use) in an open laboratory arena. While bold and timid individuals each utilized both fast and slow modes of locomotion, the amount of time spent in each mode and the duration of pauses exhibited while moving differed between the behavioural types. 
Bolder individuals spent more time moving fast, for longer durations and with shorter pauses than more timid individuals. Should these behavioural differences between behavioural types be related to risk assessment and exploration of novel environments as I suggested (in Chapter 3), these findings have important implications for understanding survivorship and fitness of personality types for animals in nature. This chapter provides the first empirical evidence of a relationship between intermittent locomotion and personality in any taxon.

In Chapter 4, I characterized the relationship between individual boldness and vulnerability to being captured by two different techniques in bluegill sunfish. First, using line angling and seining capture techniques, I caught juvenile bluegill sunfish in the wild and tested them for their boldness under standardized conditions in the laboratory. I predicted that fish caught via angling would be bolder on average than those caught haphazardly with a seine. Second, I conducted a fishing experiment in a large outdoor pool, wherein I angled for fish previously caught in the wild via seining. Here, I also predicted that bold fish would be more likely to be angled than timid fish, and that bold fish would tend to be caught in the open areas of the pool rather than near refuge. Unexpectedly, most of my findings did not support my initial predictions or suggested an opposite direction to the predicted relationship. Contrary to expectation, the more timid fish were more likely to be caught by angling than bolder fish. This inverse relationship between boldness and vulnerability to capture did not appear to be related to differences in capture stress, as there were no significant differences in plasma cortisol level in fish captured by either seining or angling when individual differences in body size were accounted for. 
Similarly, in the outdoor pool experiment, individual likelihood of capture was not related to boldness in the laboratory. However, as predicted, bolder individuals were more likely to be caught in the open, presumably riskier, area of the experimental pool rather than near refuge. While the direction of the observed relationship between boldness and capture technique differed from my initial predictions, my findings nonetheless remain important for ecologists. This study represents the first empirical evidence for a relationship between capture technique and boldness in a wild population of fish in a natural system. These findings suggest that sampling biases associated with capture technique, as postulated by Biro and Dingemanse (2009), are present and should be of concern to behavioural biologists (Beard and Essington 2000; Biro and Post 2008). Further, since fish populations are known to be susceptible to anthropogenic influences (i.e. fishing-induced evolution; Uusi-Heikkila et al. 2008), my findings suggest potentially significant ecological and evolutionary consequences for recreational/commercial angling practices in species of popular and economic interest, such as the bluegill sunfish.

In Chapter 5, I investigated whether individual differences in boldness covaried with reproductive fitness correlates in the Eastern mosquitofish. I tested wild-caught females for boldness using standardized methods and then dissected them to ascertain their relative fecundity and stages of embryonic development (i.e. stage of pregnancy). I predicted that females carrying more embryos or embryos closer to parturition would be bolder than females with fewer embryos or embryos earlier in development due to greater overall embryonic investment. As predicted, bolder individuals had a larger number of embryos (higher fecundity) relative to their body size than more timid individuals. 
However, boldness was not correlated with stage of development, but it was correlated with water column use and general activity level. These findings suggest that overall reproductive investment may influence the expression of personality traits in animals and, as such, reproductive status may provide a mechanistic explanation for some of the variation in boldness observed in wild populations, particularly during breeding and pregnancy. This study provides the first empirical evidence for a link between boldness and a reproductive fitness correlate in fishes.

\section{Implications and future research directions}

As stated in the Synthesis section above, the primary aim of my thesis research was to investigate the behavioural ecology of boldness in a variety of ecologically-relevant paradigms. Chapters 3-5 incorporated areas of significant interest to behavioural ecologists including the relationship between boldness and locomotion, reproductive fitness correlates, and unnatural selection associated with harvesting practices. Further, I also examined the across-context consistency and repeatability of boldness using the

framework of behavioural syndromes (Chapter 2). Each of my experiments represents a novel and important contribution to the field of animal personality research and offer further support for the notion that boldness is a widespread but underappreciated phenomenon that poses many important but largely unanswered questions.

There has been much debate about the contextual nature of boldness and whether it is domain-general or domain-specific (see Chapter 2). Increasingly, empirical evidence has supported both possibilities, depending on the species and population (see Réale et al. 2007). Context specificity represents a potentially fruitful avenue for future research, 
because understanding how boldness varies between behavioural contexts and how consistent these relationships are across taxa will provide insights into how boldness may evolve and how variation in this trait is maintained in animal populations. Further, the development of an experimentally 'flexible', yet ecologically relevant, manner of testing boldness in different species would greatly facilitate taxonomic comparisons that are scientifically meaningful. While such a test does not currently exist per se, quantifying individual differences in refuge emergence does appear to hold much promise as indicated by numerous studies including my own (Chapters 2-5), which have successfully used the open-arena test to quantify individual tendency to take risks in both novel and familiar environments.

While personality research has long been the realm of psychologists (Wilson et al. 1994), it is only recently that personality traits such as boldness have been of interest to behavioural ecologists. As such, it is difficult to grasp the 'big picture' when it comes to the proximate mechanisms underlying individual-level differences in boldness, as there are so many unanswered questions regarding the diverse array of physiological, behavioural and ecological mechanisms that can generate intrapopulation variation in behaviour. Additionally, while my work has provided novel insights into heretofore largely unrecognized areas of boldness research in fishes (e.g. intermittent locomotion, boldness syndromes, reproductive fitness correlates and anthropogenic selection), much of it would represent the first steps of a larger experimental program in the future. In many instances, I was able to characterize herein a relationship between boldness and another ecological trait (e.g. boldness and intermittent locomotion), but was only able to speculate as to its underlying proximate basis and the manner by which this relationship 
might have arisen. However, this type of research is often correlational and therefore does not necessarily indicate causation. Questions regarding the heritability of boldness and related traits also remain largely unanswered. To be relevant evolutionarily, individual differences in boldness should be heritable and thus subject to selection, not just an adaptive response along a population-level reaction norm.

While I observed a relationship between boldness and relative fecundity in mosquitofish (Chapter 5), I cannot state whether the observed inter-individual differences in behaviour are due solely to boldness, underlying metabolic or physiological constraints associated with pregnancy, or some combination of both behavioural and physiological factors. Similarly, I can also only speculate as to the underlying proximate basis of the relationship between boldness and intermittent locomotion (Chapter 3). Individual differences in undulatory and labriform modes of swimming and associated pauses may, independently or collectively, result from (a) individual differences in boldness, (b) underlying variation in the neural architecture associated with sensory stimuli processing, or (c) underlying differences in metabolic requirements. In the case of the relationship between boldness and activity, is it that bold fish are more active because they are more likely to take risks? Or is it that fish with higher metabolic needs are more likely to take risks to meet their physiological requirements and thus behave more boldly? As components of a future research program, I would investigate the underlying mechanistic bases of the relationships between boldness and other behavioural and morphological traits by (1) estimating the heritability of boldness and other traits (e.g. fecundity, activity) with which it is known to covary, (2) investigate potential physiological (e.g. red: white muscle index, liver glycogen) and hormonal (e.g. cortisol) factors that may 
play a role in generating variation in behaviour among individuals, and (3) investigate potential differences in the underlying neural architecture (e.g. telencephalon, optic lobes) associated with spatial memory and sensory processing that may influence behavioural traits and/or decision-making processes.

Another line of potential research possibilities could include an investigation into the relationship between ecological niche, boldness, and the aforementioned experimental paradigms (Chapters 2-5). Both sunfish and mosquitofish are relatively opportunistic generalist foragers (though niche specialization can occur; Robinson et al. 1993; Pyke 2005), which is one factor that makes them particularly invasive and well suited for dispersal into new environments. In addition, both species are prey for other larger piscivorous species of fishes (Scott and Crossman 1998; Pyke 2005). Ascertaining how boldness varies both between species and across ecological niches could be insightful, as species occupying different niches would likely be selected to respond variably depending on habitat complexity and ambient predation risk. Further, this notion of niche variation may be interesting in terms of comparisons to larger species and predators (e.g. largemouth bass) in particular, as one may predict that individuals occupying different niches would respond differently to novel stimuli (i.e. fishing lures as in Chapter 4) due to differences in their foraging habits. Further, these behaviours might vary with lifehistory stage (e.g. juvenile fish being more susceptible to predation; Godin 1997) and thus offer insight into fitness trade-offs through ontogeny as well as the inherent adaptability or inflexibility of boldness.

Boldness is a behavioural phenomenon that has many potentially important ecological, evolutionary and conservation implications. Initial theory (see Wilson 1998; 
Dall et al. 2004) suggesting that individual-level differences were largely unimportant was undoubtedly an oversimplification, which could only be justified if individual differences were predictable and having a trivial effect on ecological processes. In contrast, most recent evidence suggests boldness represents a potentially important ecological trait that exists across a wide range of taxa and is implicated in many aspects of an animal's daily behavioural repertoire. Further, owing to observed relationships between boldness and other ecologically-relevant traits, including those described for the bluegill sunfish in this thesis, the evolutionary importance of boldness remains a subject in need of further investigation. The potential for boldness to influence a variety of frequency-dependent interactions, inter- and intra-specific competition, fitness trade-offs, and therein a population's capacity to diversify and perhaps speciate, suggests that research on personality in general also represents an important field warranting further inquiry. 


\section{REFERENCES}

Allendorf FW, Hard JJ, 2009. Human-induced evolution caused by unnatural selection through harvest of wild animals. Proc Natl Acad Sci USA 106: 9987-9994.

Ashley MV, Willson MF, Pergams ORW, O'Dowd DJ, Gende SM, Brown JS, 2003. Evolutionarily enlightened management. Biol Conserv 111: 115-123.

Barber I, Hoare D, Krause J, 2000. Effects of parasites on fish behaviour: a review and evolutionary perspective. Rev Fish Biol Fish 10: 131-165.

Barros M, Alencar C, Tomaz C, 2004. Differences in aerial and terrestrial visual scanning in captive black tufted-ear marmosets (Callithrix penicillata) exposed to a novel environment. Folia Primatol 75: 85-92.

Barton BA, 2002. Stress in fishes: A diversity of responses with particular reference to changes in circulating corticosteroids. Integr Comp Biol 42: 517-525.

Beard TD, Essington TE, 2000. Effects of angling and life history processes on bluegill size structure: Insights from an individual-based model. Trans Am Fish Soc 129: $561-568$.

Bednekoff PA, Lima SL, 2002. Why are scanning patterns so variable? An overlooked question in the study of anti-predator vigilance. J Avian Biol 33: 143-149.

Bell AM, 2005. Behavioral differences between individuals and two populations of stickleback (Gasterosteus aculeatus). J Evol Biol 18: 464-473.

Bell AM, 2007. Animal personalities. Nature 447:539-540.

Bell AM, Sih A, 2007. Exposure to predation generates personality in threespined sticklebacks (Gasterosteus aculeatus). Ecol Lett 10: 828-834. 
Bell AM, Stamps JA, 2004. Development of behavioural differences between individuals and populations of sticklebacks, Gasterosteus aculeatus. Anim Behav 68: 13391348.

Biro PA, Dingemanse NJ, 2009. Sampling bias resulting from animal personality. Trends Ecol Evol 24: 66-67.

Biro PA, Post JR, 2008. Rapid depletion of genotypes with fast growth and bold personality traits from harvested fish populations. Proc Natl Acad Sci USA 105: 2919-2922.

Blumstein DT, Daniel JC, 2007. Quantifying behavior the JWatcher way. Sunderland, MA: Sinauer Associates, Inc.

Bolger T, Connolly PL, 1989. The selection of suitable indices for the measurement and analysis of fish condition. J Fish Biol 34: 171-182.

Bolnick DI, Svanback R, Fordyce JA, Yang LH, Davis JM, Hulsey CD, Forister ML, 2003. The ecology of individuals: Incidence and implications of individual specialization. Am Nat 161: 1-28.

Boon AK, Réale D, Boutin S, 2007. The interaction between personality, offspring fitness and food abundance in North American red squirrels. Ecol Lett 10: 1094-1104.

Both C, Dingemanse NJ, Drent PJ, Tinbergen JM, 2005. Pairs of extreme avian personalities have highest reproductive success. J Anim Ecol 74: 667-674.

Brana F, 2003. Morphological correlates of burst speed and field movement patterns: the behavioural adjustment of locomotion in wall lizards (Podarcis muralis). Biol J Linn Soc 80: 135-146. 
Brown C, 2001. Familiarity with the test environment improves escape responses in the crimson spotted rainbowfish, Melanotaenia duboulayi. Anim Cogn 4: 109-113.

Brown C, Braithwaite VA, 2004. Size matters: a test of boldness in eight populations of the poeciliid Brachyraphis episcopi. Anim Behav 68: 1325-1329.

Brown C, Burgess F, Braithwaite VA, 2007b. Heritable and experiential effects on boldness in a tropical poeciliid. Behav Ecol Sociobiol 62: 237-243.

Brown C, Jones F, Braithwaite V, 2005. In situ examination of boldness-shyness traits in the tropical poeciliid, Brachyraphis episcopi. Anim Behav 70: 1003-1009.

Brown C, Jones F, Braithwaite VA, 2007a. Correlation between boldness and body mass in natural populations of the poeciliid Brachyrhaphis episcopi. J Fish Biol 71: $1590-1601$.

Careau V, Thomas D, Humphries MM, Réale D, 2008. Energy metabolism and animal personality. Oikos 117: 641-653.

Carere C, van Oers K, 2004. Shy and bold great tits (Parus major): body temperature and breath rate in response to handling stress. Physiol Behav 82: 905-912.

Coble DW, 1988. Effects of angling on bluegill populations: Management implications. N Am J Fish Manag 8: 277-283.

Coleman K, Wilson DS, 1998. Shyness and boldness in pumpkinseed sunfish: individual differences are context-specific. Anim Behav 56: 927-936.

Colgan P, Gotceitas V, Frame J, 1991. Individual variation, acquisition, and reacquisition in a foraging task by juvenile bluegill sunfish (Lepomis macrochirus). J Comp Psychol 105: 78-84. 
Cooke SJ, Suski CD, 2005. Do we need species-specific guidelines for catch-and-release recreational angling to effectively conserve diverse fishery resources? Biodivers Conserv 14: 1195-1209.

Cooke SJ, Suski CD, Barthel BL, Ostrand KG, Tufts BL, Philipp DP, 2003. Injury and mortality induced by four hook types on bluegill and pumpkinseed. N Am J Fish Manag 23: 883-893.

Dall SRX, Houston AI, McNamara JM, 2004. The behavioral ecology of personality: consistent individual differences from an adaptive perspective. Ecol Lett 7: 734739.

Dewey MR, Zigler SJ, 1996. An evaluation of fluorescent elastomer for marking bluegills in experimental studies. Prog Fish-Culturist 58: 219-220.

Dingemanse NJ, Both C, Drent PJ, Tinbergen JM, 2004. Fitness consequences of avian personalities in a fluctuating environment. Proc R Soc Lond, Series B. 271: 847852.

Dingemanse NJ, Both C, Drent PJ, van Oers K, van Noordwijk AJ, 2002. Repeatability and heritability of exploratory behaviour in great tits from the wild. Anim Behav 64: 929-938.

Dingemanse NJ, Both C, van Noordwijk AJ, Rutten AL, Drent PJ, 2003. Natal dispersal and personalities in great tits (Parus major). Proc R Soc Lond, Series B 270: 741747.

Dingemanse NJ, Réale D, 2005. Natural selection and animal personality. Behaviour 142: 1159-1184. 
Dingemanse NJ, Wright J, Kazem AJN, Thomas DK, Hickling R, Dawnay N, 2007.

Behavioral syndromes differ predictably between 12 populations of three-spined stickleback. J Anim Ecol 76: 1128-1138.

Dochtermann NA, Jenkins SH, 2007. Behavioural syndromes in Merriam's kangaroo rats (Dipodomys merriami): a test of competing hypotheses. Proc R Soc Lond, Series B 274: 2343-2349.

Drent PJ, van Oers K, van Noordwijk AJ, 2003. Realized heritability of personalities in the great tit (Parus major). Proc R Soc Lond, Series B 270: 45-51.

Dugatkin LA, Alfieri MS, 2003. Boldness, behavioral inhibition and learning. Ethol Ecol Evol 15: 43-49.

Dugatkin LA, Godin J-GJ, 1992. Prey approaching predators: a cost-benefit perspective. Ann Zool Fenn 29: 233-252.

Farwell M, McLaughlin RL, 2009. Alternative foraging tactics and risk taking in brook charr (Salvelinus fontinalis). Behav Ecol 20: 913-921.

Foster SA, Endler JA (eds) 1999. Geographic variation in behavior: perspectives on evolutionary mechanisms. Oxford: Oxford University Press.

Fraser DF, Gilliam JF, Daley MJ, Le AN, Skalski GT, 2001. Explaining leptokurtic movement distributions: Intrapopulation variation in boldness and exploration. Am Nat 158: 124-135.

Frost AJ, Winrow-Giffen A, Ashley PJ, Sneddon LU, 2007. Plasticity in animal personality traits: does prior experience alter the degree of boldness? Proc R Soc Lond, Series B 274: 333-339. 
Gamperl AK, Vijayan MM, Boutilier RG, 1994. Experimental control of stress hormone levels in fishes: techniques and applications. Rev Fish Biol Fish 4: 215-255.

Gleeson TT, Hancock TV, 2001. Modeling the metabolic energetics of brief and intermittent locomotion in lizards and rodents. Am Zool 41: 211-218.

Godin J-GJ, 1997. Evading predators. In: Behavioural ecology of teleost fishes (Godin JGJ, ed). Oxford: Oxford University Press; pp. 191-236.

Godin J-GJ, Dugatkin LA, 1996. Female mating preference for bold males in the guppy, Poecilia reticulata. Proc Natl Acad Sci USA 93: 10262-10267.

Gosling SD, 2001. From mice to men: What can we learn about personality from animal research? Psychol Bull 127: 45-86.

Gotceitas V, Colgan P, 1988. Individual variation in learning by foraging juvenile bluegill sunfish (Lepomis macrochirus). J Comp Psychol 102: 294-299.

Gross MR, 1982. Sneakers, satellites and parentals - polymorphic mating strategies in North American sunfishes. Z Tierpsychol 60: 1-26.

Hancock TV, Gleeson TT, 2005. Intermittent locomotor activity that increases endurance also increases metabolic costs in the desert iguana (Dipsosaurus dorsalis). Physiol Biochem Zool 78: 163-172.

Hart A, Lendrem DW, 1984. Vigilance and scanning patterns in birds. Anim Behav 32: $1216-1224$.

Hedrick AV, 2000. Crickets with extravagant mating songs compensate for predation risk with extra caution. Proc R Soc Lond, Series B 267: 671-675.

Heino M, Godo OR, 2002. Fisheries-induced selection pressures in the context of sustainable fisheries. Bull Mar Sci 70: 639-656. 
Higham TE, 2007. Feeding, fins and braking maneuvers: locomotion during prey capture in centrarchid fishes. J Exp Biol 210: 107-117.

Jennings MJ, Claussen JE, Philipp DP, 1997. Effect of population size structure on reproductive investment of male bluegill. N Am J Fish Manag 17: 516-524.

Johnson JC, Sih A, 2005. Precopulatory sexual cannibalism in fishing spiders (Dolomedes triton): a role for behavioral syndromes. Behav Ecol Sociobiol 58: 390-396.

Jones EA, Lucey KS, Ellerby DJ, 2007. Efficiency of labriform swimming in the bluegill sunfish (Lepomis macrochirus). J Exp Biol 210: 3422-3429.

Jones KA, Godin J-GJ, 2010. Are fast explorers slow reactors? Linking personality type and anti-predator behaviour. Proc R Soc, Series B 277:625-632.

Kendall JL, Lucey KS, Jones EA, Wang J, Ellerby DJ, 2007. Mechanical and energetic factors underlying gait transitions in bluegill sunfish (Lepomis macrochirus). $\mathrm{J}$ Exp Biol 210: 4265-4271.

Kieffer JD, Colgan PW, 1992. Differences in learning by foraging juvenile pumpkinseed and bluegill sunfish in a structured habitat. Environ Biol Fishes 33: 359-366.

Koch F, Wieser W, 1983. Partitioning of energy in fish: can reduction of swimming activity compensate for the cost of production? J Exp Biol 107: 141-146.

Kortet R, Hedrick A, 2007. A behavioural syndrome in the field cricket Gryllus integer: intrasexual aggression is correlated with activity in a novel environment. Biol J Linn Soc 91: 475-482.

Kortet R, Rantala MJ, Hedrick A, 2007. Boldness in anti-predator behaviour and immune defence in field crickets. Evol Ecol Res 9: 185-197. 
Kramer DL, McLaughlin RL, 2001. The behavioral ecology of intermittent locomotion. Am Zool 41: 137-153.

Krause J, Ruxton GD, 2002. Living in groups. Oxford: Oxford University Press.

Kuparinen A, Merila J, 2007. Detecting and managing fisheries-induced evolution. Trends Ecol Evol 22: 652-659.

Lessells CM, Boag PT, 1987. Unrepeatable repeatabilities: a common mistake. Auk 104: $116-121$.

Lima SL, Dill LM, 1990. Behavioral decisions made under the risk of predation - a review and prospectus. Can J Zool 68: 619-640.

Lindström K, St Mary C, Gunnels B, Hale R, Osenberg C, Stevens S, Vonesh J, Wilson J, 2003. Reproductive investment in relation to survival risk in a livebearing fish. $\mathbf{J}$ Fish Biol 63:236-236.

Lopez P, Hawlena D, Polo V, Amo L, Martin J, 2005. Sources of individual shy-bold variations in antipredator behaviour of male Iberian rock lizards. Anim Behav 69: $1-9$.

Marsh-Matthews E, Skierkowski P, DeMarais A, 2001. Direct evidence for mother-toembryo transfer of nutrients in the livebearing fish Gambusia geiseri. Copeia 2001: 1-6.

Martin JGA, Réale D, 2008. Temperament, risk assessment and habituation to novelty in eastern chipmunks, Tamias striatus. Anim Behav 75: 309-318.

McAdam AG, Kramer DL, 1998. Vigilance as a benefit of intermittent locomotion in small mammals. Anim Behav 55: 109-117. 
McCairns RJS, Fox MG, 2004. Habitat and home range fidelity in a trophically dimorphic pumpkinseed sunfish (Lepomis gibbosus) population. Oecologia 140: 271-279.

McDougall PT, Réale D, Sol D, Reader SM, 2006. Wildlife conservation and animal temperament: causes and consequences of evolutionary change for captive, reintroduced, and wild populations. Anim Conserv $9: 39-48$.

McLaughlin RL, Grant JWA, 2001. Field examination of perceptual and energetic bases for intermittent locomotion by recently-emerged brook charr in still-water pools. Behaviour 138: 559-574.

Nakagawa S, Cuthill IC, 2007. Effect size, confidence interval and statistical significance: a practical guide for biologists. Biol Rev 82: 591-605.

Pitcher, T. J., Parrish, J. K. 1993: Functions of shoaling behaviour in teleosts. In: Behaviour of teleost fishes (Pitcher, T. J., ed.). Chapman \& Hall, New York. pp. 363-439.

Price T, Langen T, 1992. Evolution of correlated characters. Trends Ecol Evol 7:307-310.

Pyke GH, 2005. A review of the biology of Gambusia affinis and G. holbrooki. Rev Fish Biol Fish 15: 339-365.

Réale D, Festa-Bianchet M, 2003. Predator-induced natural selection on temperament in bighorn ewes. Anim Behav 65: 463-470.

Réale D, Gallant BY, Leblanc M, Festa-Bianchet M, 2000. Consistency of temperament in bighorn ewes and correlates with behaviour and life history. Anim Behav 60: $589-597$. 
Réale D, Martin J, Coltman DW, Poissant J, Festa-Bianchet M, 2009. Male personality, life-history strategies and reproductive success in a promiscuous mammal. J Evol Biol 22: 1599-1607.

Réale D, Reader SM, Sol D, McDougall PT, Dingemanse NJ, 2007. Integrating animal temperament within ecology and evolution. Biol Rev 82: 291-318.

Reaney LT, Backwell PRY, 2007. Risk-taking behavior predicts aggression and mating success in a fiddler crab. Behav Ecol 18: 521-525.

Rehage JS, Sih A, 2004. Dispersal behavior, boldness, and the link to invasiveness: a comparison of four Gambusia species. Biol Invasions 6: 379-391.

Reznick D, 1981. "Grandfather effects": The genetics of interpopulation differences in offspring size in the mosquito fish. Evolution 35: 941-953.

Rice WR, 1989. Analyzing tables of statistical tests. Evolution 43: 223-225.

Robinson BW, Wilson DS, 1994. Character release and displacement in fishes: a neglected literature. Am Nat 144: 596-627.

Robinson BW, Wilson DS, Margosian AS, Lotito PT, 1993. Ecological and morphological-differentiation of pumpkinseed sunfish in lakes without bluegill sunfish. Evol Ecol 7: 451-464.

Scott WB, Crossman EJ, 1998. Freshwater fishes of Canada. Oakville: Galt House Publications Ltd.

Sih A, 1992. Prey uncertainty and the balancing of antipredator and feeding needs. Am Nat 139: 1052-1069.

Sih A, 1997. To hide or not to hide? Refuge use in a fluctuating environment. Trends Ecol Evol 12: 375-376. 
Sih A, Bell AM, 2007. Insights from behavioural syndromes for the evolutionary genetics of personality. Eur J Personality 21:626-628.

Sih A, Bell AM, 2008. Insights for behavioral ecology from behavioral syndromes. Adv Study Behav 38: 227-281.

Sih A, Bell AM, Johnson JC, Ziemba RE, 2004. Behavioral syndromes: An integrative overview. Q Rev Biol 79: 241-277.

Sih A, Kats LB, Maurer EF, 2003. Behavioural correlations across situations and the evolution of antipredator behaviour in a sunfish-salamander system. Anim Behav 65: $29-44$.

Sinn DL, Apiolaza LA, Moltschaniwskyj NA, 2006. Heritability and fitness-related consequences of squid personality traits. J Evol Biol 19: 1437-1447.

Sinn DL, Gosling SD, Moltschaniwskyj NA, 2008. Development of shy/bold behaviour in squid: context-specific phenotypes associated with developmental plasticity. Anim Behav 75: 433-442.

Skúlason S, Smith TB, 1995. Resource polymorphisms in vertebrates. Trends Ecol Evol 10: $366-370$.

Smith BR, Blumstein DT, 2008. Fitness consequences of personality: a meta-analysis. Behav Ecol 19: 448-455.

Sneddon LU, 2003. The bold and the shy: individual differences in rainbow trout. J Fish Biol 62: 971-975.

Spotte S, 2007. Bluegills. Biology and behavior. Bethesda, MD: American Fisheries Society. 
Stamps JA, 2007. Growth-mortality tradeoffs and 'personality traits' in animals. Ecol Lett 10: $355-363$.

Stapley J, Keogh JS, 2005. Behavioral syndromes influence mating systems: floater pairs of a lizard have heavier offspring. Behav Ecol 16: 514-520.

Sundström LF, Petersson E, Hojesjo J, Johnsson JI, Jarvi T, 2004. Hatchery selection promotes boldness in newly hatched brown trout (Salmo trutta): implications for dominance. Behav Ecol 15: 192-198.

Svartberg K, Tapper I, Temrin H, Radesater T, Thorman S, 2005. Consistency of personality traits in dogs. Anim Behav 69: 283-291.

Trouilloud W, Delisle A, Kramer DL, 2004. Head raising during foraging and pausing during intermittent locomotion as components of antipredator vigilance in chipmunks. Anim Behav 67: 789-797.

Uusi-Heikkila S, Wolter C, Klefoth T, Arlinghaus R, 2008. A behavioral perspective on fishing-induced evolution. Trends Ecol Evol 23: 419-421.

van Oers K, Drent PJ, de Goede P, van Noordwijk AJ, 2004. Realized heritability and repeatability of risk-taking behaviour in relation to avian personalities. Proc R Soc Lond, Series B 271: 65-73.

van Oers K, Drent PJ, Dingemanse NJ, Kempenaers B, 2008. Personality is associated with extrapair paternity in great tits, Parus major. Anim Behav 76: 555-563.

van Oers K, Klunder M, Drent PJ, 2005. Context dependence of personalities: risk-taking behavior in a social and a nonsocial situation. Behav Ecol 16: 716-723.

Ward AJW, Thomas P, Hart PJB, Krause J, 2004. Correlates of boldness in three-spined sticklebacks (Gasterosteus aculeatus). Behav Ecol Sociobiol 55: 561-568. 
Webster MM, Ward AJW, Hart PJB, 2007. Boldness is influenced by social context in threespine sticklebacks (Gasterosteus aculeatus). Behaviour 144: 351-371.

Webster MM, Ward AJW, Hart PJB, 2009. Individual boldness affects interspecific interactions in sticklebacks. Behav Ecol Sociobiol 63: 511-520.

Wilson ADM, Godin J-GJ, 2009. Boldness and behavioral syndromes in the bluegill sunfish, Lepomis macrochirus. Behav Ecol 20: 231-237.

Wilson ADM, Godin J-GJ, 2010. Boldness and intermittent locomotion in the bluegill sunfish, Lepomis macrochirus. Behav Ecol 21: 57-62.

Wilson ADM, Godin J-GJ, Ward AJW, 2010b. Boldness and reproductive fitness correlates in the Eastern mosquitofish, Gambusia holbrooki. Ethology 116: 96104.

Wilson ADM, McLaughlin RL, 2007. Behavioural syndromes in brook charr, Salvelinus fontinalis: prey-search in the field corresponds with space use in novel laboratory situations. Anim Behav 74: 689-698.

Wilson ADM, Stevens ED, 2005. Consistency in context-specific measures of shyness and boldness in rainbow trout, Oncorhynchus mykiss. Ethology 111: 849-862.

Wilson ADM, Whattam EM, Bennett R, Visanuvimol L, Lauzon C, Bertram SM, 2010a. Behavioral correlations across activity, mating, exploration, aggression, and antipredator contexts in the European house cricket, Acheta domesticus. Behav Ecol Sociobiol 64:703-715.

Wilson DS, 1998. Adaptive individual differences within single populations. Proc R Soc Lond, Series B 353: 199-205. 
Wilson DS, Clark AB, Coleman K, Dearstyne T, 1994. Shyness and boldness in humans and other animals. Trends Ecol Evol 9: 442-446.

Wilson DS, Coleman K, Clark AB, Biederman L, 1993. Shy bold continuum in pumpkinseed sunfish (Lepomis gibbosus): an ecological study of a psychological trait. J Comp Psychol 107: 250-260.

Wolf M, van Doorn GS, Leimar O, Weissing FJ, 2007. Life-history trade-offs favour the evolution of animal personalities. Nature 447: 581-584.

Wolf M, van Doorn GS, Weissing FJ, 2008. Evolutionary emergence of responsive and unresponsive personalities. Proc Natl Acad Sci USA 105: 15825-15830.

Wright D, Nakamichi R, Krause J, Butlin RK. 2006. QTL analysis of behavioral and morphological differentiation between wild and laboratory zebrafish (Danio rerio). Behav Genet. 36: 271-284. 